DOI 10.4171/JEMS/263

Gabriele Mondello

\title{
Riemann surfaces with boundary and natural triangulations of the Teichmüller space
}

Received December 6, 2009 and in revised form April 24, 2010

\begin{abstract}
We compare some natural triangulations of the Teichmüller space of hyperbolic surfaces with geodesic boundary and of some bordifications. We adapt Scannell-Wolf's proof to show that grafting semi-infinite cylinders at the ends of hyperbolic surfaces with fixed boundary lengths is a homeomorphism. This way, we construct a family of equivariant triangulations of the Teichmüller space of punctured surfaces that interpolates between Bowditch-Epstein-Penner's (using the spine construction) and Harer-Mumford-Thurston's (using Strebel differentials). Finally, we show (adapting arguments of Dumas) that on a fixed punctured surface, when the triangulation approaches HMT's, the associated Strebel differential is well-approximated by the Schwarzian of the associated projective structure and by the Hopf differential of the collapsing map.
\end{abstract}

\section{Introduction}

\subsection{Overview}

The aim of this paper is to compare two different ways of triangulating the Teichmüller space $\mathcal{T}(R, x)$ of conformal structures on a compact oriented surface $R$ with distinct ordered marked points $x=\left(x_{1}, \ldots, x_{n}\right)$. Starting with $\left[f: R \rightarrow R^{\prime}\right] \in \mathcal{T}(R, x)$ and a collection of weights $\underline{\tilde{p}}=\left(\tilde{p}_{1}, \ldots, \tilde{p}_{n}\right) \in \Delta^{n-1}$, both constructions produce a ribbon graph $G$ embedded in the punctured surface $\dot{R}=R \backslash x$ as a deformation retract, together with a positive weight for each edge. A suitable completion of the space of such weighted graphs can be identified with the topological realization of the arc complex $\mathfrak{A}(R, x)$ via Poincaré-Lefschetz duality on $(R, x)$ (see for instance [Mon09b]), which is the simplicial complex of (isotopy classes of) systems of (homotopically nontrivial, pairwise nonhomotopic) arcs that join couples of marked points and that admit representatives with disjoint interior ([Har86], [BE88], [Lo095]).

Thus, both constructions provide a $\Gamma(R, x)$-equivariant homeomorphism $\mathcal{T}(R, x) \times$ $\Delta^{n-1} \rightarrow\left|\mathfrak{A}^{\circ}(R, x)\right|$, where $\Gamma(R, x)=\pi_{0} \operatorname{Diff}_{+}(R, x)$ is the mapping class group of $(R, x)$ and $\mathfrak{A}^{\circ}(R, x) \subset \mathfrak{A}(R, x)$ consists of proper systems of $\operatorname{arcs} \boldsymbol{A}=\left\{\alpha_{0}, \ldots, \alpha_{k}\right\}$, namely such that $\dot{R} \backslash\left(\alpha_{0} \cup \cdots \cup \alpha_{k}\right)$ is a disjoint union of discs and pointed discs. In fact, properness of $\boldsymbol{A}$ is exactly equivalent to its dual ribbon graph being a deformation retract of $\dot{R}$.

G. Mondello: Dipartimento di Matematica, Università di Roma "La Sapienza", Piazzale Aldo Moro, 2, 00185 Roma, Italy; e-mail: mondello@mat.uniroma1.it 
The HMT construction (due to Harer, Mumford and Thurston) appears in [Har86]. It uses Strebel's result [Str67] on existence and uniqueness of a meromorphic quadratic differential $\varphi$ on a Riemann surface $R$ with prescribed residues $p$ at $x$ to decompose $\dot{R}$ into a disjoint union of semi-infinite $|\varphi|$-flat cylinders (one for each puncture $x_{i}$ with $\tilde{p}_{i}>0$ ), that are identified along a critical graph $G$ which inherits this way a metric. The length of each edge of $G$ will be its weight.

The BEP construction (due to Bowditch-Epstein [BE88] and Penner [Pen87]) uses the unique hyperbolic metric on the punctured Riemann surface $\dot{R}$. Given a (projectively) decorated surface, that is, a hyperbolic surface $\dot{R}$ with cusps plus a weight $\underline{\tilde{p}} \in \Delta^{n-1}$, there are disjoint embedded horoballs of circumference $\tilde{p}_{1}, \ldots, \tilde{p}_{n}$ at the $n$ cusps of $\dot{R}$. Removing the horoballs, we obtain a truncated surface $R^{\operatorname{tr}}$ with boundary, on which the function "distance from the boundary" is well-defined. The critical locus of this function is a spine $G$ embedded in $R^{\text {tr }} \subset \dot{R}$ as a deformation retract and with geodesic edges, whose horocyclic lengths provide the associated weights.

Both constructions share similar properties of homogeneity and real-analyticity (see [HM79] and [Pen87]) and they also enjoy some good compatibility with the WeilPetersson symplectic structure on $\mathcal{T}(R, x)$, as explained later.

In this paper, we will interpolate these two constructions using the Teichmüller space $\mathcal{T}(S)$ of hyperbolic surfaces with geodesic boundary (see also [Luo07]), where $S$ is a surface with boundary endowed with a homotopy equivalence $S \hookrightarrow \dot{R}$. The spine construction works perfectly on such surfaces, even when they are nodal (which is the content of Theorem 3.16, and it can be easily seen to reduce to the BEP case as the boundary lengths $\underline{p}=\left(p_{1}, \ldots, p_{n}\right)=r \underline{\tilde{p}}$ become infinitesimal (see also [Mon09c]). Also, the Weil-Petersson Poisson structure can be explicitly determined, thus providing a generalization of Penner's formula [Pen92].

Thus, the limit $r:=p_{1}+\cdots+p_{n} \rightarrow 0$ is completely understood in terms of hyperbolic surfaces with cusps and Bers's augmented Teichmüller space [Ber74].

On the other hand, our comprehension of the limit $r \rightarrow \infty$ involves flat surfaces and it shares some similarities with Thurston's compactification [FLP79] of the Teichmüller space. In fact, the space of proper arc systems $\left|\mathfrak{A}^{\circ}(S)\right|$ naturally embeds in the space of projective measured laminations. From a symplectic point of view, the Weil-Petersson structure admits a precise limit as $r \rightarrow \infty$, after a suitable normalization, which agrees with Kontsevich's piecewise linear symplectic form on $|\mathfrak{A}(S)|$ defined in [Kon92] (see [Mon09c]).

To give a more geometric framework to these limiting considerations, we produce a few different bordifications of the Teichmüller space $\mathcal{T}(S)$ of a surface $S$ with boundary, whose quotients by the mapping class group $\Gamma(S)$ give different compactifications of the moduli space. A convenient bordification from the point of view of the Weil-Petersson Poisson structure is the extended Teichmüller space $\widetilde{\mathcal{T}}(S)$; whereas the most suitable one for triangulations and spine constructions is the bordification of $\operatorname{arcs} \overline{\mathcal{T}}^{a}(S)$ (described in Theorem 3.16, whose definition looks a bit like Thurston's but with some relevant differences (for instance, we use $t$-lengths related to hyperbolic collars instead of hyperbolic lengths). It is reasonable to believe that careful iterated blow-ups of $\overline{\mathcal{T}}^{a}(S)$ along its sin- 
gular locus would produce finer bordifications of $\mathcal{T}(S)$ in the spirit of [Lo095] (see also [MP07]).

In order to explicitly link the HMT and BEP constructions, we construct a family of isotopic triangulations of $\mathcal{T}(R, x) \times \Delta^{n-1}$, parametrized by $r \in[0, \infty]$, that coincides with BEP for $r=0$ and with HMT for $r=\infty$ (and this is the content of Corollary 5.6. In particular, we prove that, for every complex structure on $\dot{R}$ and every $(\underline{\tilde{p}}, r) \in \Delta^{n-1} \times[0, \infty]$, there exists a unique projective structure $\mathcal{P}(\dot{R}, r \underline{\tilde{p}})$ on $\dot{R}$, whose associated Thurston metric has flat cylindrical ends (with circumferences $r \tilde{p}$ ) and a hyperbolic core. Rescaling the lengths by a factor $1 / r$, we recognize that at $r=\infty$ the hyperbolic core shrinks to a graph $G$ and the metric on the grafted surface is of the type $|\varphi|$, where $\varphi$ is a Strebel differential. This result (Theorem 5.4) can be restated in terms of infinite grafting at the ends of a hyperbolic surface with geodesic boundary and the proof adapts arguments of Scannell-Wolf [SW02].

Finally, we show that, for large $r$, two results of Dumas [Dum06], [Dum07b] for compact surfaces still hold (Theorem 5.12). The first one says that, for $r$ large, the Strebel differential $\varphi$ is well-approximated in $L_{\text {loc }}^{1}(\dot{R})$ by the Hopf differential of the collapsing map associated to $\mathcal{P}(\dot{R}, r \underline{\tilde{p}})$, that is the quadratic differential which is $d z^{2}$ on the flat cylinders $S^{1} \times[0, \infty)$ and is zero on the hyperbolic part. The second result says that $\varphi$ is also well-approximated by the Schwarzian derivative of the projective structure $\mathcal{P}(\dot{R}, r \underline{p})$.

\subsection{Organization of the paper}

In Section 2, we recall the definitions of the Teichmüller space $\mathcal{T}(S)$ of a surface $S$ with boundary and its augmentation, hyperbolic boundary lengths $\underline{p}=\left(p_{1}, \ldots, p_{n}\right)$, mapping class group, Weil-Petersson form and arc complex.

Incidentally, we discuss three topologies on the arc complex of $S$ : the standard coherent one, the metric one (which we will regularly use) and the one coming from the natural embedding in the space of measured laminations on the doubled surface $d S$; and we show that they agree on the locus of proper arc systems but they may differ in general.

Section 3 begins by introducing some coordinate systems on the Teichmüller space associated to a system of arcs, for instance the $a$-lengths (hyperbolic lengths of the arcs), the $t$-lengths (hyperbolic widths of the estimated collars of the arcs) and the actual widths $w$.

Coordinates associated to arc systems do not allow us to rebuild the whole hyperbolic surface in general, but only certain "visible" components. Hence, the triangulation maps will always identify nodal surfaces whose visible components are isomorphic.

Our first main result is Theorem 3.16 the spine construction continuously extends to the locus of nodal surfaces with finite boundary length and to the locus of projectively decorated surfaces, there reproducing BEP's triangulation (namely, the normalized widths reduce to the $\lambda$-lengths as $\underline{p} \rightarrow 0$ ). The proof of the continuity, which is crucial, is postponed to Appendix B

Then we define the bordification of $\operatorname{arcs} \overline{\mathcal{T}}^{a}(S)$, which is the space that we are really able to triangulate: it is the closure of $\mathcal{T}(S)$ in the (projective) space of $t$-length functions. This construction bears some similarity to Thurston's, even though we use a stronger topology on the $t$-length functions. 
Our second main result (Theorem 3.17) is an explicit description of $\overline{\mathcal{T}}^{a}$ : we construct a homeomorphism $\Phi:|\mathfrak{A}(S)| \times[0, \infty]_{r} \rightarrow \overline{\mathcal{T}}^{a}(S)$ that interpolates between the natural inclusion $|\mathfrak{A}(S)| \hookrightarrow \overline{\mathcal{T}}^{a}(S)$ at $r:=p_{1}+\cdots+p_{n}=\infty$ and the inverse of the $\lambda$-lengths at $r=0$. This means that the $t$-lengths behave like $w$ for $r$ large and like $\lambda^{-1}$ for $r$ small.

We finally extend the augmented Teichmüller space by adding a copy of the arc complex at $r=\infty$.

In Section 4 we recall an explicit formula $[$ Mon09c] for the Weil-Petersson Poisson structure in terms of lengths of arcs (Theorem 4.3) and we describe how it reduces to the formulae of Penner [Pen92] at $r=0$ and Kontsevich [Kon92] at $r=\infty$.

Moreover, we describe how to extend the previous triangulations to the case of a surface with boundary $S$ and a marked point on each boundary component, which is somewhat analogous to having a closed pointed surface together with a nonzero tangent vector at every marked point.

In Section 5 we relate hyperbolic surfaces with boundary to punctured surfaces. Here the infinite grafting map plays a key role.

First we define the ribbon graph dual to an arc system and we describe the HMT triangulation and its extension to nodal surfaces using the flat metrics associated to Strebel differentials.

Then we introduce the infinite grafting, that takes a hyperbolic surface with geodesic boundary $\Sigma$ and returns a punctured surface $\operatorname{gr}_{\infty}(\Sigma)$ obtained by gluing a semi-infinite flat cylinder at each boundary component of $\Sigma$.

Our main result is Theorem 5.4 for every nonnegative $\underline{p}$, the map $\mathrm{gr}_{\infty}$ gives a homeomorphism between the space of hyperbolic surfaces with boundary lengths $\underline{p}$ and the Teichmüller space of closed surfaces with $n$ marked points.

Composing the inverse of $\mathrm{gr}_{\infty}$ with the spine construction, we obtain a (continuous) family of triangulations of the Teichmüller space of closed surfaces with $n$ marked points, which reduce to BEP's for infinitesimal $\underline{p}$ and to HMT's for infinite $\underline{p}$ (Corollary 5.6).

The continuity at infinity of $\mathrm{gr}_{\infty}$ requires some explicit computations and it is proven in Appendix $\mathrm{D}$, for the injectivity of $\mathrm{gr}_{\infty}$, we simply adapt arguments of Scannell-Wolf [SW02] to our situation.

It would be interesting to investigate whether the techniques of Appendix $D$ could be employed to attack the following problem (which I believe was raised by Dennis Sullivan).

Problem. Fix the parameter p. Composing BEP's with the inverse of HMT's triangulation maps, we obtain a self-homeomorphism $\mathfrak{S}$ of the Teichmüller space. Given a point $q \in \mathcal{T}(S)$, consider the Teichmüller distance between $q$ and $\mathfrak{S}(q)$. Is this distance uniformly bounded?

At the end of Section 5, we investigate the degeneration as $r \rightarrow \infty$ of the projective structure induced by the infinite grafting procedure, adapting arguments of Dumas [Dum06], [Dum07b].

We stress that the proofs of these last results and of the local injectivity of the grafting map assume the knowledge of the papers [SW02], [Dum06] and [Dum07b]. 
In Appendix A we recall the Collar Lemma and a useful convergence criterion in the augmented Teichmüller space. In Appendix C we prove two simple topological lemmata that are needed in the main proofs.

After the Appendix, there is a table of the most used symbols.

\section{Preliminaries}

\subsection{Double of a surface with boundary}

A nodal surface with boundary is a compact, Hausdorff topological space $S$ with countable basis in which every $q \in S$ has an open neighbourhood $U_{q}$ such that $\left(U_{q}, q\right)$ is homeomorphic to either: $(\mathbb{C}, 0)$, and then $q$ is called a smooth internal point; $(\{z \in \mathbb{C} \mid \operatorname{Im}(z)$ $\geq 0\}, 0)$, and then $q$ is called a boundary point; or $\left(\left\{(z, w) \in \mathbb{C}^{2} \mid z w=0\right\}, 0\right)$, and then $q$ is called a node. The smooth locus of a nodal surface is the complement of its nodes, and the boundary locus $\partial S$ is the union of its boundary points.

A nodal surface with marked points is a nodal surface $S$ together with an ordered subset $x=\left(x_{1}, \ldots, x_{n}\right)$ of distinct smooth interior points of $S$. We will write $S^{\circ}:=$ $S \backslash(\partial S \cup x)$.

We will say that a (nodal) surface $S$ is closed if it has no boundary and no marked points.

By slight abuse of language, we define a hyperbolic metric on $(S, x)$ to be a complete metric $g$ of finite volume on the smooth locus of the punctured surface $\dot{S}:=S \backslash x$ of constant curvature -1 , such that $\partial S$ is geodesic. Notice that $\dot{S}$ contains the possible nodes of $S$, and $g$ acquires cusps at the marked points and at the nodes (which are points of $S$ ).

Given a (possibly nodal) surface $S$ with boundary and/or marked points, we can construct its double $d S$ in the following way. Let $S^{\prime}$ be another copy of $S$, with opposite orientation, and let $q^{\prime} \in S^{\prime}$ the point corresponding to $q \in S$. Define $d S$ to be $S \amalg S^{\prime} / \sim$, where $\sim$ is the equivalence relation generated by $q \sim q^{\prime}$ for every $q \in \partial S$ and $x_{i} \sim x_{i}^{\prime}$ for every $i$. Clearly, $d S$ is closed and it is smooth whenever $S$ has no nodes and no marked points.

$d S$ can be oriented so that the natural embedding $\iota: S \hookrightarrow d S$ is orientation-preserving. Moreover, $d S$ comes naturally equipped with an orientation-reversing involution $\sigma$ that fixes $\iota(\partial S)$ and the nodes $\iota(x)$ and such that $d S / \sigma \cong S$. If $S$ is hyperbolic, then $d S$ can be given a hyperbolic metric such that $\iota$ and $\sigma$ are isometries.

Clearly, on $d S$ there is a correspondence between complex structures and hyperbolic metrics and, in fact, $\sigma$-invariant hyperbolic metrics correspond to complex structures such that $\sigma$ is anti-holomorphic. Thus, the datum of a hyperbolic metric with geodesic boundary on $S$ is equivalent to that of a complex structure on $S$ such that $\partial S$ is totally real.

\subsection{Teichmüller space}

Let $S$ be a smooth surface with $n \geq 0$ boundary components $C_{1}, \ldots, C_{n}$ such that $\chi(S)<0$. 
Definition 2.1. An S-marked hyperbolic surface is an orientation-preserving map $f$ : $S \rightarrow \Sigma$ of (smooth) surfaces that may shrink boundary components of $S$ to cuspidal points of $\Sigma$ and that is a diffeomorphism everywhere else.

Two $S$-marked surfaces $f_{1}: S \rightarrow \Sigma_{1}$ and $f_{2}: S \rightarrow \Sigma_{2}$ are equivalent if there exists an isometry $h: \Sigma_{1} \rightarrow \Sigma_{2}$ such that $h \circ f_{1}$ is homotopic to $f_{2}$.

Definition 2.2. Denote by $\check{\mathcal{T}}(S)$ the set of equivalence classes of $S$-marked hyperbolic surfaces and let $\mathcal{T}(S) \subset \check{\mathcal{T}}(S)$ be the locus of surfaces $\Sigma$ with no cusps.

For every point $[f: S \rightarrow \Sigma]$ of $\check{\mathcal{T}}(S)$, we consider a universal covering space $u: \widetilde{\Sigma^{\circ}} \rightarrow \Sigma^{\circ}$ and we endow $\widetilde{\Sigma^{\circ}}$ with the pull-back metric. Thus, a locally isometric developing map dev $: \widetilde{\Sigma^{\circ}} \rightarrow \mathbb{H}$ is unique up to the action of $\operatorname{PSL}_{2}(\mathbb{R}) \cong \operatorname{Aut}(\underset{\widetilde{I}}{)})$. Using the isomorphism $f_{*}: \pi_{1}(S) \rightarrow \pi_{1}\left(\Sigma^{\circ}\right)$, the fundamental group $\pi_{1}(S)$ acts on $\widetilde{\Sigma^{\circ}}$ via isometries and so we obtain a representation $\rho: \pi_{1}(S) \rightarrow \mathrm{PSL}_{2}(\mathbb{R})$. It can be easily shown that $\rho$ is discrete and faithful and that $\Sigma$ can be recovered as Bers-Nielsen kernel (see [Ber76]) of the complete surface $\mathbb{H} / \rho\left(\pi_{1}(S)\right)$. Hence, the map $\check{\mathcal{T}}(S) \rightarrow \operatorname{Rep}\left(\pi_{1}(S), \operatorname{PSL}_{2}(\mathbb{R})\right)$ is injective, where $\operatorname{Rep}\left(\pi_{1}(S), \operatorname{PSL}_{2}(\mathbb{R})\right)=\operatorname{Hom}\left(\pi_{1}(S), \operatorname{PSL}_{2}(\mathbb{R})\right) / \operatorname{PSL}_{2}(\mathbb{R})$ and $\operatorname{PSL}_{2}(\mathbb{R})$ acts on the space of homomorphisms by conjugation. Thus $\operatorname{Rep}\left(\pi_{1}(S), \operatorname{PSL}_{2}(\mathbb{R})\right)$ has a natural topology and we put on $\check{\mathcal{T}}(S)$ the subspace topology.

Definition 2.3. The locus $\mathcal{T}(S) \subset \check{\mathcal{T}}(S)$ of surfaces $\Sigma$ with no cusps is called the Teichmüller space.

Let $\gamma=\left\{C_{1}, \ldots, C_{n}, \gamma_{1}, \ldots, \gamma_{3 g-3+n}\right\}$ be a maximal system of disjoint simple closed curves of $S$ such that no $\gamma_{i}$ is contractible and no couple $\left\{\gamma_{i}, \gamma_{j}\right\}$ or $\left\{\gamma_{i}, C_{j}\right\}$ bounds a cylinder. The system $\gamma$ induces a pair of pants decomposition of $S$, that is, $S^{\circ} \backslash \bigcup_{i} \gamma_{i}=P_{1} \cup \cdots \cup P_{2 g-2+n}$, and each $P_{h}$ is a pair of pants (i.e. a surface homeomorphic to $\mathbb{C} \backslash\{0,1\})$.

Given $[f: S \rightarrow \Sigma] \in \mathcal{T}(S)$, we can define $\ell_{i}(f)$ to be the length of the unique geodesic curve isotopic to $f\left(\gamma_{i}\right)$. Fenchel-Nielsen twist deformations along $\gamma_{1}, \ldots$, $\gamma_{3 g-3+n}$ act freely and transitively on the fibres of $\ell_{\gamma}: \check{\mathcal{T}}(S) \rightarrow \mathbb{R}_{+}^{3 g-3+n} \times \mathbb{R}_{\geq 0}^{n}$, which thus becomes an affine $\mathbb{R}^{3 g-3+n}$-fibration. In fact, as the base is contractible, this fibration is trivializable. This means that one can define global twist parameters $\tau_{i}(f)$ on $\breve{\mathcal{T}}(S)$ that coordinatize the fibres of $\ell_{\boldsymbol{\gamma}}$ (see [Abi80], [BP92] or [Rat06], for instance). The resulting global functions $\left(p_{j}, \ell_{i}, \tau_{i}\right)$ are called Fenchel-Nielsen coordinates.

The boundary length map $\mathcal{L}: \check{\mathcal{T}}(S) \rightarrow \mathbb{R}_{\geq 0}^{n}$ is defined as $\mathcal{L}([f])=\left(p_{1}, \ldots, p_{n}\right)$ and we write $\check{\mathcal{T}}(S)(\underline{p}):=\mathcal{L}^{-1}(\underline{p})$ for $\underline{p} \in \mathbb{R}_{\geq 0}^{n}$. Thus, $\mathcal{T}(S)=\check{\mathcal{T}}(S)\left(\mathbb{R}_{+}^{n}\right)$.

\subsection{The arc complex}

Assume that the smooth surface $S$ has at least a boundary component or a marked point. Let $\mathcal{A}(S)$ be the set of all nontrivial isotopy classes of simple $\operatorname{arcs} \alpha \subset S$ with $\alpha^{\circ} \subset S^{\circ}$ and endpoints at $\partial S$ or at the marked points of $S$. A $k$-system of arcs $\boldsymbol{A}=\left\{\alpha_{1}, \ldots, \alpha_{k}\right\}$ $\subset \mathcal{A}(S)$ is a subset of arcs of $S$ that admit representatives which can intersect only at the 
marked points. The system $\boldsymbol{A}$ fills (resp. quasi-fills) $S$ if $S \backslash \boldsymbol{A}:=S \backslash \bigcup_{\alpha_{i} \in \boldsymbol{A}} \alpha_{i}$ is a disjoint union of discs (resp. discs, pointed discs and annuli homotopic to boundary components); $\boldsymbol{A}$ is also called proper if it quasi-fills $S$ ([Lo095]).

Definition 2.4 ([Har86]). The complex of $\operatorname{arcs} \mathfrak{A}(S)$ of a surface $S$ with boundary and/or marked points is the simplicial complex whose $k$-simplices are $(k+1)$-systems of arcs on $S$.

Denote by $|\mathfrak{A}(S)|$ the geometric realization of $\mathfrak{A}(S)$, which comes endowed with two natural topologies. The coherent topology is the finest topology that makes the realization of all simplicial maps continuous. The metric topology is induced by the path metric, for which every $k$-simplex is isometric to the standard $\Delta^{k} \subset \mathbb{R}^{k+1}$ and every attachment map is a local isometry.

We will denote by $\mathfrak{A}^{\circ}(S) \subset \mathfrak{A}(S)$ the subset of proper systems of arcs, which is the complement of a lower-dimensional simplicial subcomplex, and by $\left|\mathfrak{A}^{\circ}(S)\right| \subset|\mathfrak{A}(S)|$ the locus of weighted proper systems, which is open and dense. The metric topology on $|\mathfrak{A}(S)|$ is coarser than the coherent one, but they agree where the complex is locally finite, namely on $\left|\mathfrak{A}^{\circ}(S)\right|$. Endowing all realizations with the metric topology will be our standard choice, unless differently specified.

Notation. If $\boldsymbol{A}=\left\{\alpha_{1}, \ldots, \alpha_{k}\right\} \in \mathfrak{A}(S)$, then a point $w \in|\boldsymbol{A}| \subset|\mathfrak{A}(S)|$ is a formal sum $w=\sum_{i} w_{i} \alpha_{i}$ such that $w_{i} \geq 0$ and $\sum_{i} w_{i}=1$, which can also be seen as a function $w: \mathcal{A}(S) \rightarrow \mathbb{R}$ supported on $\boldsymbol{A}$.

Let $\mathcal{M L}(d S)$ be the space of measured laminations on $d S$ and let $\mathbb{P} \mathcal{M L}(d S)$ be its projectivization (see [FLP79]). Denote by $\mathcal{M L}(d S)^{\sigma}$ and $\mathbb{P} \mathcal{M L}(d S)^{\sigma}$ the fixed loci under the involution $\sigma$. As an arc $\alpha_{i} \subset S$ doubles to a simple closed curve in $d S$, there is a set-theoretic inclusion $D:|\mathfrak{A}(S)| \hookrightarrow \mathbb{P} \mathcal{M L}(d S)^{\sigma}$.

Lemma 2.5. (a) The subspace topology induced by D is strictly coarser than the coherent topology and strictly finer than the metric topology.

(b) The image of $D$ is neither open nor closed.

(c) The restriction of $D$ to $\left|\mathfrak{H}^{\circ}(S)\right|$ (where both topologies coincide) is a homeomorphism onto an open subset of $\mathbb{P} \mathcal{M L}(d S)^{\sigma}$.

Proof. To prove (a), fix a maximal system of arcs $\boldsymbol{A}=\left\{\alpha_{i}\right\}$ and let $D \alpha_{i} \subset d S$ be the double of $\alpha_{i}$. If $w^{(m)}=\sum_{i} w_{i}^{(m)} \alpha_{i} \rightarrow w=\sum_{i} w_{i} \alpha_{i}$, then $w_{i}^{(m)} \rightarrow w_{i}$ for every $i$ and so $D\left(w^{(m)}\right)=\sum_{i} w_{i}^{(m)}\left[D \alpha_{i}\right] \rightarrow \sum_{i} w_{i}\left[D \alpha_{i}\right]=D(w)$. This shows that the coherent topology is finer than the subspace topology.

To compare the subspace and metric topologies, pick $w=\sum w_{i} \alpha_{i} \in|\mathfrak{A}(S)|$ and $w^{(m)}=\sum v_{j}^{(m)} \beta_{j}^{(m)} \in|\mathfrak{A}(S)|$ such that $D\left(w^{(m)}\right) \rightarrow D(w)$ in $\mathbb{P} \mathcal{M L}(S)$. Complete $\boldsymbol{A}=\left\{\alpha_{i}\right\}$ to a maximal system of arcs $\boldsymbol{A}^{\prime}=\left\{\alpha_{i}\right\} \cup\left\{\alpha_{h}^{\prime}\right\}$ and define $w^{\prime}=w+\delta \sum_{h} \alpha_{h}^{\prime}$, where $\delta=\min _{i} w_{i}$.

For every $m$, write $w^{(m)}$ as a sum $\tilde{w}^{(m)}+\hat{w}^{(m)}$ of two nonnegative multi-arcs in such a way that all arcs in the support of $\hat{w}^{(m)} \operatorname{cross} \boldsymbol{A}^{\prime}$ and that $i\left(\tilde{w}^{(m)}, w^{\prime}\right)=0$. 
Let $t_{m}$ be the sum of the weights in $\hat{w}^{(m)}$, so that $d\left(w, w^{(m)}\right) \leq d\left(w, \tilde{w}^{(m)}\right)+t_{m}$, where $d$ is the path metric on $|\mathfrak{A}(S)|$. Because

$$
t_{m} \delta \leq i\left(\hat{w}^{(m)}, w^{\prime}\right)=i\left(w^{(m)}, w^{\prime}\right) \rightarrow i\left(w, w^{\prime}\right)=0
$$

it follows that $t_{m} \rightarrow 0$. Moreover, $\tilde{w}^{(m)}$ has support contained in $\boldsymbol{A}^{\prime}$ and so $w^{(m)} \rightarrow w$ in $|\mathfrak{A}(S)|$ for the metric topology. This proves that the subspace topology is finer than the metric topology.

Now, pick two disjoint arcs $\{\alpha, \beta\} \in \mathfrak{A}(S)$ and a simple closed curve $\gamma$ (possibly, a boundary component of $S$ ) such that $\alpha \cap \gamma=\emptyset$ and $i(\beta, \gamma)=1$. Consider the weighted arc systems $w^{(m)}=\left(1-\varepsilon_{m}\right) \alpha+\varepsilon_{m} T_{\gamma}^{m}(\beta)$ in $|\mathfrak{A}(S)|$, where $T_{\gamma}^{m}$ is the $k$-uple Dehn twist along $\gamma$. If $\varepsilon_{m}>0$ and $\varepsilon_{m} \rightarrow 0$, then $w^{(m)} \rightarrow \alpha$ for the metric topology but $w^{(m)}$ is divergent for the coherent topology.

One can check that $D\left(w^{(m)}\right) \rightarrow[\alpha]$ for $\varepsilon_{m}=1 / m^{2}$ and that $D\left(w^{(m)}\right) \rightarrow[\gamma]$ for $\varepsilon_{m}=1 / \sqrt{m}$. As a consequence, the metric topology is strictly coarser than the subspace topology, which is strictly coarser than the coherent topology; moreover, the image of $D$ is not closed.

Clearly, the image of $D$ is not open: if $\alpha$ is an arc and $\gamma \subset S$ is simple closed curve disjoint from $\alpha$, then $[D \alpha]=D(\alpha)$ and $\left[D \alpha+m^{-1} D \gamma\right] \rightarrow[D \alpha]$, but $\left[D \alpha+m^{-1} D \gamma\right]$ does not belong to the image of $D$. This proves (b).

Finally, notice that $D\left(\left|\mathfrak{A}^{\circ}(S)\right|\right)$ is open by invariance of domain, because $\left|\mathfrak{A}^{\circ}(S)\right|$ and $\mathbb{P} \mathcal{M L}(d S)^{\sigma}$ are topological manifolds of the same dimension (the former result seems due to Whitney [HM79] and the latter to Thurston [FLP79]). Hence, (c) follows from (a).

\subsection{Weil-Petersson metric}

Let $S$ be a smooth surface with (possibly empty) boundary $\partial S=C_{1} \cup \cdots \cup C_{n}$ and $\chi(S)<0$. Let $[f: S \rightarrow \Sigma]$ be a point of $\mathcal{T}(S)$.

Define $\mathcal{Q}_{\Sigma}$ to be the real vector space of holomorphic quadratic differentials $q(z) d z^{2}$ whose restriction to $\partial \Sigma$ is real. Similarly, define the real vector space of harmonic Beltrami differentials as $\mathcal{B}_{\Sigma}:=\left\{\mu=\mu(z) d \bar{z} / d z=\bar{\varphi} d s^{-2} \mid \varphi \in \mathcal{Q}_{\Sigma}\right\}$, where $d s^{2}$ is the hyperbolic metric on $\Sigma$.

It is well-known that the tangent space $T_{[f]} \mathcal{T}(S)$ to $\mathcal{T}(S)$ at $[f]$ can be identified with $\mathcal{B}_{\Sigma}$ and, similarly, the cotangent space $T_{[f]}^{*} \mathcal{T}(S) \cong \mathcal{Q}_{\Sigma}$. The natural coupling is given by

$$
\begin{aligned}
\mathcal{B}_{\Sigma} \times \mathcal{Q}_{\Sigma} & \rightarrow \mathbb{C}, \\
(\mu, \varphi) & \mapsto \int_{\Sigma} \mu \varphi .
\end{aligned}
$$

Definition 2.6. The Weil-Petersson pairing on $T_{[f]} \mathcal{T}(S)$ is defined as

$$
h(\mu, v):=\int_{\Sigma} \mu \bar{\nu} d s^{2} \quad \text { with } \mu, v \in \mathcal{B}_{\Sigma}
$$

Writing $h=g+i \omega$, we call $g$ the Weil-Petersson Riemannian metric and $\omega$ the WeilPetersson form. For $T_{[f]}^{*} \mathcal{T}(S)$, we similarly have $h^{\vee}(\varphi, \psi):=\int_{\Sigma} \varphi \bar{\psi} d s^{-2}$ with $\varphi, \psi$ $\in \mathcal{Q}_{\Sigma}$. The Weil-Petersson Poisson structure is $\eta:=\operatorname{Im}\left(h^{\vee}\right)$. 
It follows from the definition that the doubling map $D: \mathcal{T}(S) \rightarrow \mathcal{T}(d S)$ is a homothety of factor 2 onto a real Lagrangian submanifold of $\mathcal{T}(d S)$.

From Wolpert's work [Wol83], we learn that $\omega=\sum_{i=1}^{N} d \ell_{i} \wedge d \tau_{i}$, where $\left(p_{1}, \ldots, p_{n}\right.$, $\left.\ell_{1}, \tau_{1}, \ldots, \ell_{N}, \tau_{N}\right)$ are Fenchel-Nielsen coordinates, and so $\omega$ is degenerate whenever $S$ has boundary. In this case, the symplectic leaves are the $\mathcal{T}(S)(\underline{p})$, which are not totally geodesic subspaces for $g$ (unless $p_{1}=\cdots=p_{n}=0$ and the boundary components degenerate to cusps).

Remark 2.7. $\mathcal{T}(S)$ is naturally a complex manifold if $S$ is closed. In this case, $\omega$ and $\eta$ are nondegenerate and the Weil-Petersson metric is Kähler (see [Ahl61]).

\subsection{Augmented Teichmüller space}

Let $S$ be a smooth surface with boundary $\partial S=C_{1} \cup \cdots \cup C_{n}$ such that $\chi(S)<0$.

Definition 2.8. An $S$-marked stable surface $\Sigma$ is a hyperbolic surface possibly with geodesic boundary components, cusps and nodes plus an isotopy class of maps $f$ : $S \rightarrow \Sigma$ that shrinks some boundary components of $S$ to the cusps of $\Sigma$, some loops of $S$ to the nodes of $\Sigma$ and is an orientation-preserving diffeomorphism elsewhere.

We say that $f_{1}: S \rightarrow \Sigma_{1}$ and $f_{2}: S \rightarrow \Sigma_{2}$ are equivalent if there exists an isometry $h: \Sigma_{1} \rightarrow \Sigma_{2}$ such that $h \circ f_{1}$ is homotopic to $f_{2}$. We denote by $\overline{\mathcal{T}}(S)$ the set of stable $S$-marked surfaces up to equivalence. Clearly, $\mathcal{T}(S) \subset \check{\mathcal{T}}(S) \subset \overline{\mathcal{T}}(S)$.

To describe the topology of $\overline{\mathcal{T}}(S)$ around a stable surface $[f: S \rightarrow \Sigma$ ] with $k$ cusps and $d$ nodes, choose a system of curves $\left\{C_{1}, \ldots, C_{n}, \gamma_{1}, \ldots, \gamma_{N}\right\}$ on $S$ (with $N=$ $3 g-3+n)$ adapted to $f$, i.e. such that $f^{-1}\left(v_{j}\right)=\gamma_{j}$ for each of the nodes $v_{1}, \ldots, v_{d}$ of $\Sigma$. Clearly, the Fenchel-Nielsen coordinates $\left(p_{1}, \ldots, p_{n}, \ell_{1}, \tau_{1}, \ldots, \ell_{N}, \tau_{N}\right)$ extend over $[f]$, with the exception of $\tau_{1}(f), \ldots, \tau_{d}(f)$, which are not defined.

We declare that the sequence $\left\{\left[f_{m}: S \rightarrow \Sigma_{m}\right]\right\} \subset \overline{\mathcal{T}}(S)$ converges to $[f]$ if $p_{i}\left(f_{m}\right)$ $\rightarrow p_{i}(f)$ for $1 \leq i \leq n, \ell_{j}\left(f_{m}\right) \rightarrow \ell_{j}(f)$ for $1 \leq j \leq N$ and $\tau_{j}\left(f_{m}\right) \rightarrow \tau_{j}(f)$ for $d+1 \leq j \leq N$.

Definition 2.9. With the above topology, $\overline{\mathcal{T}}(S)$ is called the augmented Teichmüller space (see [Ber74]).

By definition, the boundary length map extends by continuity to $\mathcal{L}: \overline{\mathcal{T}}(S) \rightarrow \mathbb{R}_{\geq 0}^{n}$ and we denote by $\overline{\mathcal{T}}(S)\left(\underline{p)}\right.$ the fibre $\mathcal{L}^{-1}(\underline{p})$. We will write $\|\underline{p}\|$ for $p_{1}+\cdots+p_{n}$ and $\|\mathcal{L}(f)\|$ for the $L^{1}$ norm of $\mathcal{L}(f)$.

Notice that the Weil-Petersson pairing diverges in directions transverse to $\partial \mathcal{T}(S)$. However, the divergence is so mild that points of $\partial \mathcal{T}(S)$ are at finite distance from points in the interior (see [Mas76]). In fact, for every $\underline{p} \in \mathbb{R}_{\geq 0}^{n}$ the augmented $\overline{\mathcal{T}}(S)(\underline{p})$ is the completion of $\mathcal{T}(S)(\underline{p})$ with respect to the Weil-Petersson metric.

Remark 2.10. According to our definition, if $S$ has nonempty boundary, then $\overline{\mathcal{T}}(S)$ is not WP-complete and in fact the image of $\overline{\mathcal{T}}(S)$ inside $\overline{\mathcal{T}}(d S)$ under the doubling map is not closed because it misses all surfaces with boundaries of infinite length. 


\subsection{The moduli space}

Let $S$ be a smooth surface of genus $g$ with boundary components $C_{1}, \ldots, C_{n}$ such that $2-2 g-n=\chi(S)<0$. The augmented Teichmüller space $\overline{\mathcal{T}}(S)$ carries a natural right action of the group $\operatorname{Diff}_{+}(S)$ of orientation-preserving diffeomorphisms of $S$ that send $C_{i}$ to $C_{i}$ for every $i=1, \ldots, n$ :

$$
\begin{aligned}
& \overline{\mathcal{T}}(S) \times \operatorname{Diff}_{+}(S) \rightarrow \overline{\mathcal{T}}(S) \\
& ([f: S \rightarrow \Sigma], h) \mapsto[f \circ h: S \rightarrow \Sigma] .
\end{aligned}
$$

Clearly, the connected component $\operatorname{Diff}_{0}(S)$ of the identity acts trivially on $\overline{\mathcal{T}}(S)$.

Definition 2.11. The mapping class group of $S$ is the quotient

$$
\Gamma(S):=\operatorname{Diff}_{+}(S) / \operatorname{Diff}_{0}(S)=\pi_{0} \operatorname{Diff}_{+}(S) .
$$

The quotient $\overline{\mathcal{M}}(S):=\overline{\mathcal{T}}(S) / \Gamma(S)$ is the moduli space of stable hyperbolic surfaces of genus $g$ with $n$ (ordered) boundary components.

The quotient map $\pi: \overline{\mathcal{T}}(S) \rightarrow \overline{\mathcal{M}}(S)$ can be identified with the forgetful map $[f: S \rightarrow \Sigma] \mapsto[\Sigma]$. Moreover, we can identify the stabilizer $\operatorname{Stab}_{[\Sigma]}(\Gamma(S))$ with the group $\mathrm{IsO}_{+}(\Sigma)$ of orientation-preserving isometries of $\Sigma$, which is finite.

$\overline{\mathcal{M}}(S)$ can be given a natural structure of orbifold (with corners), called the FenchelNielsen smooth structure. Let $[f: S \rightarrow \Sigma]$ be a point of $\overline{\mathcal{T}}(S)$ and let $\left(p_{1}, \ldots, p_{n}, \ell_{1}\right.$, $\left.\tau_{1}, \ldots, \ell_{N}, \tau_{N}\right)$ be Fenchel-Nielsen coordinates adapted to $f$. A local chart for (the Fenchel-Nielsen smooth structure of) $\overline{\mathcal{M}}(S)$ around $[\Sigma]$ is given by

$$
\begin{aligned}
\mathbb{R}_{\geq 0}^{n} \times \mathbb{C}^{3 g-3} & \rightarrow \overline{\mathcal{M}}(S) \\
(\underline{p}, \underline{z}) & \mapsto \Sigma(\underline{p}, \underline{z}),
\end{aligned}
$$

where $\left[f^{\prime}: S \rightarrow \Sigma(p, \underline{z})\right]$ is the point of $\overline{\mathcal{T}}(S)$ with coordinates $\left(p_{1}, \ldots, p_{n}, \ell_{1}, \tau_{1}, \ldots\right.$, $\left.\ell_{N}, \tau_{N}\right)$ with $\ell_{j}=\left|\overline{z_{j}}\right|$ and $\tau_{j}=\left|z_{j}\right| \arg \left(z_{j}\right) / 2 \pi$.

Remark 2.12. As shown by Wolpert [Wol85], the smooth structure on $\partial \mathcal{M}(S)=$ $\overline{\mathcal{M}}(S) \backslash \mathcal{M}(S)$ coming from Fenchel-Nielsen coordinates and the one coming from algebraic geometry (for instance, see [DM69] or [AC09]) are not the same.

We can identify the (co)tangent space to $\mathcal{T}(S)$ at $[f: S \rightarrow \Sigma]$ with the (co)tangent space to $\mathcal{M}(S)$ at $[\Sigma]$. It follows by its very definition that the Weil-Petersson metric and the boundary length map descends to $\mathcal{M}(S)$ and that $\overline{\mathcal{M}}(S)(\underline{p})$ is the metric completion of $\mathcal{M}(S)(\underline{p})$ for every $\underline{p} \in \mathbb{R}_{\geq 0}^{n}$.

The group Diff $+(S)$ also acts on $\mathfrak{A}(S)$ as $\boldsymbol{A} \cdot h:=h^{-1}(\boldsymbol{A})$, and so on $|\mathfrak{A}(S)|$ preserving the subspace $\left|\mathfrak{A}^{\circ}(S)\right|$. Moreover, this action factors through $\Gamma(S)$. We recall the following simple fact.

Lemma 2.13. $|\mathfrak{A}(S)| / \Gamma(S)$ is compact. 
Proof. It is sufficient to notice the following facts:

- the above action may not be strictly simplicial, but it is on the second barycentric subdivision $\mathfrak{A}(S)^{\prime \prime}$,

- consequently, the action is continuous,

- $\mathfrak{A}(S) / \Gamma(S)$ is a finite set and so is $\mathfrak{A}(S)^{\prime \prime} / \Gamma(S)$.

\section{Triangulations}

\subsection{Systems of arcs and widths}

Let $S$ be a smooth surface of genus $g$ with boundary components $C_{1}, \ldots, C_{n}$ such that $\chi(S)<0$. Let $\boldsymbol{A}=\left\{\alpha_{1}, \ldots, \alpha_{N}\right\} \in \mathfrak{A}^{\circ}(S)$ be a maximal system of arcs on $S$ (so that $N=6 g-6+3 n)$.

Fix a point $[f: S \rightarrow \Sigma]$ in $\mathcal{T}(S)$. For every $i=1, \ldots, N$ there exists a unique geodesic arc on $\Sigma$ in the isotopy class of $f\left(\alpha_{i}\right)$ that meets $\partial \Sigma$ perpendicularly and which we will still denote by $f\left(\alpha_{i}\right)$; denote by $a_{i}=\ell_{\alpha_{i}}(f)$ its length and let $s_{i}=\cosh \left(a_{i} / 2\right)$. Notice that $\Sigma \backslash f(\boldsymbol{A})$ is a disjoint union of right-angled hexagons $\left\{H_{1}, \ldots, H_{4 g-4+2 n}\right\}$, so that the following is immediate (see also [Ush99], [Mon09c]).

Lemma 3.1. The maps $a_{\boldsymbol{A}}: \mathcal{T}(S) \rightarrow \mathbb{R}_{+}^{\boldsymbol{A}}$ and $s_{\boldsymbol{A}}: \mathcal{T}(S) \rightarrow \mathbb{R}_{+}^{\boldsymbol{A}}$ given by $a_{\boldsymbol{A}}=$ $\left(a_{1}, \ldots, a_{N}\right)$ and $s_{\boldsymbol{A}}=\left(s_{1}, \ldots, s_{N}\right)$ are real-analytic diffeomorphisms.

Let $H$ be such a right-angled hexagon and let $\left(\vec{\alpha}_{i}, \vec{\alpha}_{j}, \vec{\alpha}_{k}\right)$ be the cyclic set of oriented arcs that bound $H$, so that $\partial H=\vec{\alpha}_{i} * \vec{\alpha}_{j} * \vec{\alpha}_{k}$. If $\vec{\alpha}_{x}, \vec{\alpha}_{y}$ are oriented arcs with endpoint on the same boundary component $C$, denote by $d\left(\vec{\alpha}_{x}, \vec{\alpha}_{y}\right)$ the length of the portion of $C$ running from the endpoint of $\vec{\alpha}_{x}$ to the endpoint of $\vec{\alpha}_{y}$ along the positive direction of $C$.

Define $w_{\boldsymbol{A}}\left(\vec{\alpha}_{i}\right)=\frac{1}{2}\left[d\left(\vec{\alpha}_{i}, \overleftarrow{\alpha}_{j}\right)+d\left(\vec{\alpha}_{k}, \overleftarrow{\alpha}_{i}\right)-d\left(\vec{\alpha}_{j}, \overleftarrow{\alpha}_{k}\right)\right]$, where $\overleftarrow{\alpha}_{x}$ is the oriented arc obtained from $\vec{\alpha}_{x}$ by switching its orientation.

Definition 3.2. For every $\alpha_{i} \in \boldsymbol{A}$, the width of $\alpha_{i}$ associated to $[f]$ is $w_{\boldsymbol{A}}\left(\alpha_{i}\right)=$ $w_{\boldsymbol{A}}\left(\vec{\alpha}_{i}\right)+w_{\boldsymbol{A}}\left(\overleftarrow{\alpha}_{i}\right)$.

\subsection{The $t$-coordinates}

Let $S$ be a surface as in the previous section.

Definition 3.3. The $t$ (ransverse)-length of an $\operatorname{arc} \alpha$ at $[f]$ is $t_{\alpha}(f):=T\left(\ell_{\alpha}(f)\right)$, where $T(x):=2 \operatorname{arcsinh}(1 / \sinh (x / 2))$.

Notice that $T(x):[0, \infty] \rightarrow[0, \infty]$ is a decreasing function of $x$ (similar to the width of the collar of a closed curve of length $x$ provided by Lemma A.1. Moreover, $T$ is involutive, $T(x) \approx 4 e^{-x / 2}$ as $x \rightarrow \infty$ and $T(x) \approx 2 \log (4 / x)$ as $x \rightarrow 0$.

Back to the $t$-length, the following lemma reduces to a statement about hyperbolic hexagons with right angles. 
Lemma 3.4. For every maximal system A of arcs,

$$
\begin{aligned}
t_{\boldsymbol{A}}: \check{\mathcal{T}}(S) & \rightarrow \quad \mathbb{R}_{\geq 0}^{\boldsymbol{A}} \\
{[f] } & \mapsto\left(t_{\alpha_{1}}(f), \ldots, t_{\alpha_{N}}(f)\right)
\end{aligned}
$$

is a continuous map that restricts to a real-analytic diffeomorphism $\mathcal{T}(S) \rightarrow \mathbb{R}_{+}^{\boldsymbol{A}}$. Moreover, for every $[f] \in \check{\mathcal{T}}(S)$ with $\mathcal{L}(f) \neq 0$, there exists an $\boldsymbol{A}$ such that $t_{\boldsymbol{A}}$ is a system of coordinates around $[f]$.

Consequently, the $t$-length map $\check{\mathcal{T}}(S) \times \mathcal{A}(S) \rightarrow \mathbb{R}_{\geq 0}$ defined as $(f, \alpha) \mapsto t_{\alpha}(f)$ gives an injection

$$
\begin{aligned}
j: \mathcal{T}(S) & \rightarrow \mathbb{P}(\mathcal{A}(S)) \times[0, \infty] \\
{[f] } & \mapsto\left(\left[t_{\bullet}(f)\right],\left\|t_{\bullet}(f)\right\|_{\infty}\right),
\end{aligned}
$$

where $L^{\infty}(\mathcal{A}(S))$ is the $\mathbb{R}_{+}$-cone of bounded maps $t: \mathcal{A}(S) \rightarrow \mathbb{R}_{\geq 0}$ and $\mathbb{P}(\mathcal{A}(S))$ is its projectivization.

Notice that $\mathbb{P}(\mathcal{A}(S))$ has a metric induced from the unit sphere of $L^{\infty}(\mathcal{A}(S))$ and that $\Gamma(S)$ acts on $\mathbb{P}(\mathcal{A}(S))$ by permuting some coordinates. Thus, $\mathbb{P}(\mathcal{A}(S)) \times[0, \infty]$ has a $\Gamma(S)$-invariant metric.

Fact 3.5. $j$ is continuous.

This will follow from Proposition 3.18

Definition 3.6. Call the closure $\overline{\mathcal{T}}^{a}(S)$ of $\mathcal{T}(S)$ in $\mathbb{P}(\mathcal{A}(S)) \times[0, \infty]$ the bordification of arcs. By the "finite part" of $\overline{\mathcal{T}}^{a}(S)$ we will mean $\overline{\mathcal{T}}^{a}(S) \cap \mathbb{P}(\mathcal{A}(S)) \times[0, \infty)$. Define the compactification of arcs to be the quotient $\overline{\mathcal{M}}^{a}(S):=\overline{\mathcal{T}}^{a}(S) / \Gamma(S)$.

We will give an explicit description of the boundary points in $\overline{\mathcal{T}}^{a}(S)$ and we will show that $\overline{\mathcal{M}}^{a}(S)$ is Hausdorff and compact.

\subsection{The spine construction}

Let $S$ be a smooth surface with boundary $\partial S=C_{1} \cup \cdots \cup C_{n}$ such that $\chi(S)<0$ and let $[f: S \rightarrow \Sigma$ ] be a point in $\mathcal{T}(S)$.

The valence $\operatorname{val}(p)$ of a point $p \in \Sigma$ is the number of paths from $p$ to $\partial \Sigma$ of minimal length.

Definition 3.7. The spine of $\Sigma$ is the locus $\operatorname{Sp}(\Sigma)$ of points of $\Sigma$ of valence at least 2 .

One can easily show that $\operatorname{Sp}(\Sigma)=V \cup E$ is a one-dimensional CW-complex embedded in $\Sigma$, where $V=\operatorname{val}^{-1}([3, \infty))$ is a finite set of points, called vertices, and $E=\mathrm{val}^{-1}(2)$ is a disjoint union of finitely many (open) geodesic arcs, called edges.

For every edge $E_{i} \subset E$ of $\operatorname{Sp}(\Sigma)$, we can define a dual arc $\alpha_{i}$ in the following way. Pick $p \in E_{i}$ and denote by $\gamma_{1}$ and $\gamma_{2}$ the two shortest paths that join $p$ to $\partial \Sigma$. Then $\alpha_{i}$ is 
the shortest arc in the homotopy class (with endpoints on $\partial \Sigma$ ) of $\gamma_{1}^{-1} * \gamma_{2}$. Let the spinal arc system $\boldsymbol{A}_{\mathrm{sp}}(\Sigma)$ be the system of arcs dual to the edges of $\operatorname{Sp}(\Sigma)$, which is proper because $\Sigma$ retracts by deformation onto $\operatorname{Sp}(\Sigma)$ just flowing away from the boundary.

Even if the spinal arc system is not maximal, widths $w_{\mathrm{sp}}$ can be associated to $\boldsymbol{A}_{\mathrm{sp}}(\Sigma)$ in the following way. For every oriented $\operatorname{arc} \vec{\alpha}_{i} \in \boldsymbol{A}_{\mathrm{sp}}(\Sigma)$ ending at $y_{i} \in C_{m}$, orient the dual edge $E_{i}$ in such a way that $\left(\vec{E}_{i}, \vec{\alpha}_{i}\right)$ is positively oriented and call $v$ the starting point of $\vec{E}_{i}$. Every point of $E_{i}$ has exactly two projections, that is, two closest points in $\partial \Sigma$; the endpoint of $\vec{\alpha}_{i}$ selects only one of these, which belongs to $C_{m}$. Denote by $v^{\prime} \in C_{m}$ the projection of $v$ determined by $\vec{\alpha}_{i}$. Define $w_{\mathrm{sp}}\left(\vec{\alpha}_{i}\right)$ to be the distance with sign $d_{C_{m}}\left(y_{i}, v^{\prime}\right)$ along $C_{m}$, which is certainly positive if $\alpha_{i}$ and $E_{i}$ intersect, but might be negative otherwise. However, the sum $w_{\mathrm{sp}}\left(\alpha_{i}\right)=w_{\mathrm{sp}}\left(\vec{\alpha}_{i}\right)+w_{\mathrm{sp}}\left(\overleftarrow{\alpha}_{i}\right)$ is always positive, being the length of either of the two projections of $E_{i}$.

Example 3.8. In Figure 1, we have $\vec{\alpha}_{i}=\overrightarrow{z_{i} y_{i}}, v^{\prime}=f_{k}$ and $w_{\mathrm{sp}}\left(\vec{\alpha}_{i}\right)>0$.

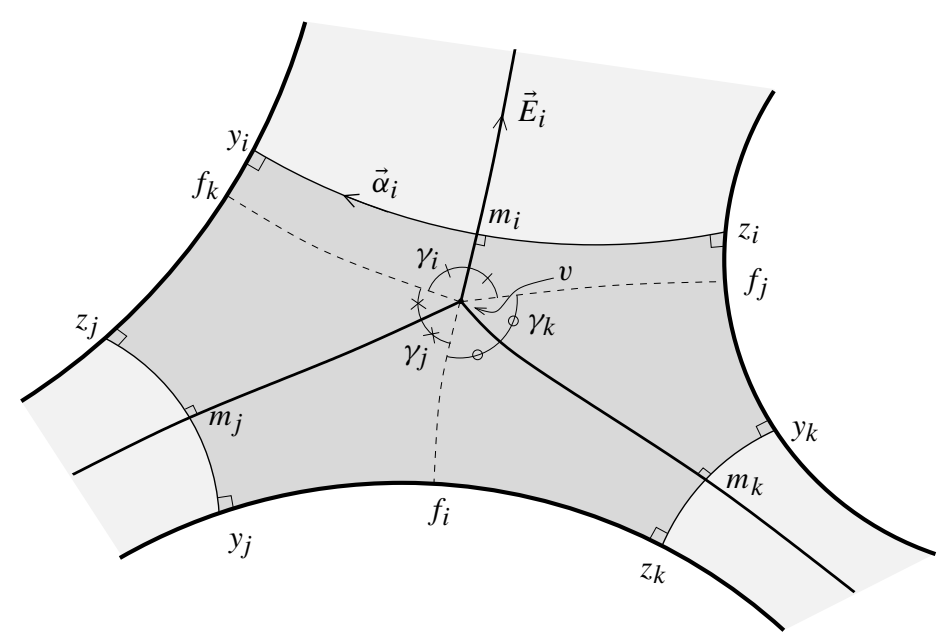

Fig. 1. Geometry of the spine close to a trivalent vertex.

Theorem 3.9 (Ushijima [Ush99]). Given a hyperbolic surface with nonempty boundary $\Sigma$, let $\mathfrak{A}(\Sigma)_{+}$be the set of all maximal systems of arcs $\boldsymbol{A}$ such that $w_{\boldsymbol{A}}\left(\alpha_{i}\right) \geq 0$ for all $\alpha_{i} \in \boldsymbol{A}$. Then $\mathfrak{A}(\Sigma)_{+}$is nonempty and the intersection of all systems in $\mathfrak{A}(\Sigma)_{+}$is exactly $\boldsymbol{A}_{\mathrm{sp}}(\Sigma)$. Moreover, $w_{\mathrm{sp}}(\alpha)=w_{\boldsymbol{A}}(\alpha)>0$ for all $\alpha \in \boldsymbol{A}_{\mathrm{sp}}(\Sigma)$ and all $\boldsymbol{A} \in \mathfrak{A}(\Sigma)_{+}$.

Remark 3.10. Let $H \subset \Sigma$ be a right-angled hexagon, bounded by $\left(\vec{\alpha}_{i}, \vec{\alpha}_{j}, \vec{\alpha}_{k}\right)$, and set $\gamma_{i}=\gamma\left(\vec{\alpha}_{i}\right)$ as in Figure 1. An easy computation [Mon09c] shows that

$$
\sinh \left(w_{\mathrm{sp}}\left(\vec{\alpha}_{i}\right)\right) \sinh \left(a_{i} / 2\right)=\cos \left(\gamma_{i}\right)=\frac{s_{j}^{2}+s_{k}^{2}-s_{i}^{2}}{2 s_{j} s_{k}}
$$


and so

$$
\sinh \left(w_{\mathrm{sp}}\left(\vec{\alpha}_{i}\right)\right)=\frac{s_{j}^{2}+s_{k}^{2}-s_{i}^{2}}{2 s_{j} s_{k} \sqrt{s_{i}^{2}-1}} \quad \text { and } \quad w_{\mathrm{sp}}\left(\vec{\alpha}_{i}\right) \leq \frac{1}{2} t_{\alpha_{i}}
$$

Theorem 3.11 (Luo [Luo07]). Given a smooth surface $S$ with nonempty boundary and $\chi(S)<0$, the map

$$
\begin{aligned}
& \boldsymbol{W}: \quad \mathcal{T}(S) \quad \rightarrow\left|\mathfrak{A}^{\circ}(S)\right| \times \mathbb{R}_{+} \\
& {[f: S \rightarrow \Sigma] \mapsto \quad f^{*} w_{\mathrm{sp}}}
\end{aligned}
$$

is a $\Gamma(S)$-equivariant homeomorphism.

Notice that the construction extends to $\check{\mathcal{T}}(S) \backslash \check{\mathcal{T}}(S)(0)$ but the locus $\check{\mathcal{T}}(S)(0)$ of surfaces with $n$ cusps is problematic, because the function "distance from the boundary $\partial \Sigma$ " diverges everywhere on $\Sigma$. This can be easily fixed by considering the real blow-up $\mathrm{Bl}_{0} \check{\mathcal{T}}(S)$ of $\check{\mathcal{T}}(S)$ along $\breve{\mathcal{T}}(0)$. The exceptional locus can be identified with the space of

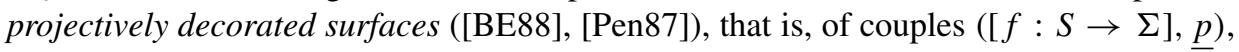
where $[f]$ is an $S$-marked hyperbolic surface with $n$ cusps and $\underline{p} \in \Delta^{n-1} \cong \mathbb{P}\left(\mathbb{R}_{\geq 0}^{n}\right)$ is a ray of weights (the decoration).

We recall the following two simple facts. The precise definitions of $\lambda$-lengths and simplicial coordinates can be found in Appendix B

Lemma 3.12 ([Mon09c] $)$. For every maximal system of arcs $\boldsymbol{A}$ (of cardinality $N=6 \mathrm{~g}-$ $6+3 n)$, the associated $t$-lengths extend to a real-analytic map

$$
t_{\boldsymbol{A}}: \mathrm{Bl}_{0} \check{\mathcal{T}}(S) \rightarrow \Delta^{N-1} \times[0, \infty) .
$$

On the exceptional locus, the projectivized t-lengths are inverses to the projectivized $\lambda$-lengths (see [Pen87] and Definition B.1). Thus, $t_{A}$ gives a system of coordinates on $\left[\check{\mathcal{T}}(S)(0) \times\left(\Delta^{n-1}\right)^{\circ}\right] \cup \mathcal{T}(S)$. Moreover, for every $(f, \underline{p}) \in \check{\mathcal{T}}(S)(0) \times \partial \Delta^{n-1}$ there exists an $\boldsymbol{A}$ such that $t_{\boldsymbol{A}}$ gives a chart around $(f, \underline{p})$.

Theorem 3.13 ([Mon09c]). The map $\boldsymbol{W}$ extends to a $\Gamma(S)$-equivariant homeomorphism

$$
\check{\boldsymbol{W}}: \mathrm{Bl}_{0} \check{\mathcal{T}}(S) \rightarrow\left|\mathfrak{A}^{\circ}(S)\right| \times[0, \infty) .
$$

On the exceptional locus, the projectivized widths coincide with the projectived simplicial coordinates (as they are called in [Pen87]; in [BE88] they are called $\vartheta$-coordinates) and so there $\breve{W}$ coincides with BEP's homeomorphism.

\subsection{Spines of stable surfaces}

Notice that the spine construction extends to stable hyperbolic surfaces $\Sigma$ (blowing up the locus of surfaces with $n$ cusps), discarding the components of $\Sigma$ where the distance 
from $\partial \Sigma$ is infinite. As a consequence, the weighted arc system we can produce does not allow us to reconstruct the full surface, but just a visible portion of it.

Definition 3.14. Let $\Sigma$ be a stable hyperbolic surface with boundary (and possibly cusps) or let $(\Sigma, p)$ be a stable (projectively) decorated surface. A component of $\Sigma$ is called visible if it contains a boundary circle or a positively weighted cusp. Denote by $\Sigma_{+}$the visible subsurface of $\Sigma$, that is, the union of the smooth points of all visible components. Two points $\left[f_{1}: S \rightarrow \Sigma_{1}\right]$ and $\left[f_{2}: S \rightarrow \Sigma_{2}\right]$ of $\widehat{\mathcal{T}}(S):=\mathrm{Bl}_{0} \overline{\mathcal{T}}(S)$ are visibly equivalent, $\left[f_{1}\right] \sim_{\text {vis }}\left[f_{2}\right]$, if there exists a third point $[f: S \rightarrow \Sigma]$ and maps $h_{i}: \Sigma \rightarrow \Sigma_{i}$ for $i=1,2$ such that $h_{i}$ restricts to an isometry $\Sigma_{+} \rightarrow \Sigma_{i,+}$ and $h_{i} \circ f \simeq f_{i}$ for $i=1,2$.

The spine $\operatorname{Sp}(\Sigma)$ of a stable hyperbolic surface $\Sigma$ with geodesic boundary (or with weighted cusps) can only be defined inside $\bar{\Sigma}_{+}$, so that its dual system of $\operatorname{arcs} \boldsymbol{A}_{\mathrm{sp}}(\Sigma)$ will be contained in $\bar{\Sigma}_{+}$too. Given a marking $\left[f: S \rightarrow \Sigma\right.$ ], we will write $S_{+}=f^{-1}\left(\Sigma_{+}\right)$, so that $\bar{S}_{+}$will be the maximal subsurface of $S$ (unique up to isotopy), quasi-filled by $f^{-1}\left(\boldsymbol{A}_{\mathrm{sp}}(\Sigma)\right)$, which carries positive weights $f^{*} w_{\mathrm{sp}}$.

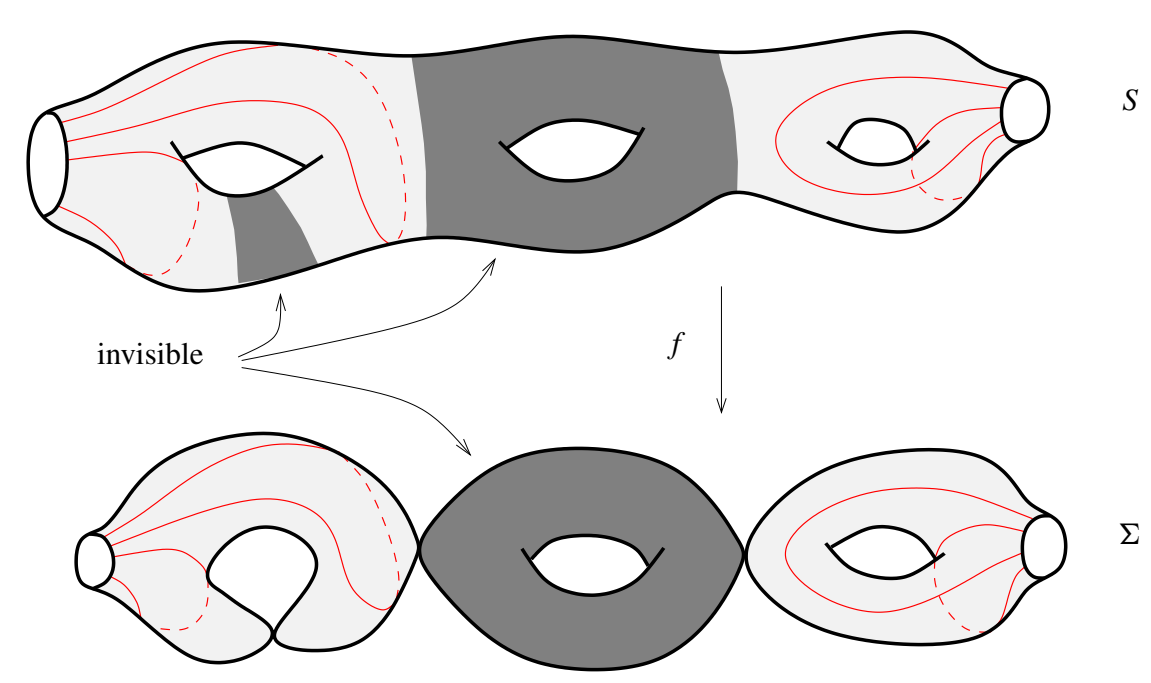

Fig. 2. An example of topological type associated to an arc system.

Conversely, given a system of $\operatorname{arcs} \boldsymbol{A} \in \mathfrak{A}(S)$, the visible subsurface $S_{+}$associated to $\boldsymbol{A}$ is the isotopy class of maximal open subsurfaces embedded in $S^{\circ}$ such that $S_{+} \simeq \bar{S}_{+}$ and $\boldsymbol{A}$ is contained in $\bar{S}_{+}$as a proper system of arcs. More concretely, $\bar{S}_{+}$is the union of a closed tubular neighbourhood of $\boldsymbol{A}$ and all components of $S \backslash \boldsymbol{A}$ which are discs or annuli isotopic to some end of $S \backslash \boldsymbol{A}$. If $\Sigma$ is obtained from $S$ by collapsing the boundary components of $\bar{S}_{+}$and the possible resulting two-noded spheres to nodes of $\Sigma$, then we obtain an isotopy class of maps $f: S \rightarrow \Sigma$, which depends only on $\boldsymbol{A}$. We will refer to this map, or just to $\Sigma$ if we work in the moduli space, as the topological type of $\boldsymbol{A}$ (see Figure 2). 
Given weights $w \in|\boldsymbol{A}|^{\circ} \times[0, \infty)$, the components of $\bar{\Sigma}_{+}=f\left(\bar{S}_{+}\right)$are quasifilled by the arc system $f(\boldsymbol{A})$; because of Theorem 3.13 , they can be given a hyperbolic metric such that $f(\boldsymbol{A})$ is its spinal arc system with weights $f_{*}(w)$. When no confusion is possible, we will still denote by $[f: S \rightarrow \Sigma$ ] the class of visibly equivalent $S$-marked stable surfaces determined by $f$.

This construction defines a $\Gamma(S)$-equivariant extension of the previous $\boldsymbol{W}^{-1}$,

$$
\widehat{\boldsymbol{W}}^{-1}:|\mathfrak{A}(S)| \times[0, \infty) \rightarrow \widehat{\mathcal{T}}^{\mathrm{vis}}(S),
$$

where $\widehat{\mathcal{T}}^{\text {vis }}(S)=\widehat{\mathcal{T}}(S) / \sim_{\text {vis }}$. Observe that $\overline{\mathcal{T}}(S)$ is a metric space with the Weil-Petersson metric, $\widehat{\mathcal{T}}(S)$ inherits a $\Gamma(S)$-equivariant metric from its natural embedding inside $\overline{\mathcal{T}}(S) \times \Delta^{n-1} \times[0, \infty)$ and $\widehat{\mathcal{T}}^{\text {vis }}(S)$ can be endowed with a $\Gamma(S)$-equivariant quotient metric defined as

$$
d_{\widehat{\mathcal{T}} \text { vis }}([f],[g]):=\inf _{k}\left\{\sum_{i=0}^{k} d_{\widehat{\mathcal{T}}}\left(\left[f_{i}\right],\left[g_{i}\right]\right) \mid f_{0} \sim_{\text {vis }} f, g_{i} \sim_{\text {vis }} f_{i+1}, g_{k} \sim_{\text {vis }} g\right\} .
$$

The argument above shows that $\widehat{\boldsymbol{W}}^{-1}$ is bijective. As already noticed in [BE88] and [Lo095], the map $\widehat{\boldsymbol{W}}$ is not continuous if $|\mathfrak{A}(S)|$ is endowed with the coherent topology.

Remark 3.15. $|\mathfrak{A}(S)|$ is locally finite at $w \Leftrightarrow \boldsymbol{A}=\operatorname{supp}(w)$ is a proper system of $\operatorname{arcs} \Leftrightarrow$ $w$ has a countable fundamental system of coherent neighbourhoods. Moreover, a sequence converges for the coherent topology if and only if it is eventually in a fixed closed simplex and there it converges in the Euclidean topology.

The discontinuity of $\widehat{\boldsymbol{W}}$ at $\partial \mathcal{T}(S)$ with respect to the coherent topology can be seen as follows. Consider a marked surface $[f: S \rightarrow \Sigma$ ] with a node $f(\gamma)=q \in \Sigma$ such that not all the boundary components of $\Sigma$ are cusps and denote by $\boldsymbol{A}$ a maximal system of arcs of $S$ such that $\widehat{\boldsymbol{W}}(f) \in|\boldsymbol{A}| \times \mathbb{R}_{+}$. Choose a sequence $\left[f_{m}: S \rightarrow \Sigma_{m}\right]$ with $\widehat{\boldsymbol{W}}\left(f_{m}\right)$ contained in $|\boldsymbol{A}|^{\circ} \times \mathbb{R}_{+}$and such that $\left[f_{m}\right] \rightarrow[f]$. If $T_{\gamma}$ is the right Dehn twist along $\gamma$ and $f_{m}^{\prime}=f_{m} \circ T_{\gamma}^{m}$, then $\left[f_{m}^{\prime}\right]$ still converges to $[f]$. On the other hand, the $\widehat{\boldsymbol{W}}\left(f_{m}^{\prime}\right)$ 's all belong to the interior of distinct maximal simplices of $|\mathfrak{A}(S)|$ and so the sequence $\widehat{\boldsymbol{W}}\left(f_{m}^{\prime}\right)$ is divergent for the coherent topology.

The correct solution (see [BE88]), which we will adopt without further notice, is to equip $|\mathfrak{A}(S)|$ with the metric topology, whose importance will also be clear in the proof of Lemma C.1

Theorem 3.16. The $\Gamma(S)$-equivariant natural extension

$$
\widehat{\boldsymbol{W}}: \widehat{\mathcal{T}}^{\mathrm{vis}}(S) \rightarrow|\mathfrak{A}(S)| \times[0, \infty)
$$

is a homeomorphism.

Proof. We know that $\widehat{\boldsymbol{W}}$ is bijective. The continuity of $\widehat{\boldsymbol{W}}$ is dealt with in Lemma B.4. In order to prove that $\widehat{W}$ is a homeomorphism, it is sufficient to show that so is the induced map

where $\widehat{\mathcal{M}}^{\text {vis }}(S)=\widehat{\mathcal{T}}^{\text {vis }}(S) / \Gamma(S)$.

$$
\widehat{\boldsymbol{W}}^{\prime}: \widehat{\mathcal{M}}^{\mathrm{vis}}(S) \rightarrow(|\mathfrak{A}(S)| / \Gamma(S)) \times[0, \infty)
$$


In fact, $\widehat{\mathcal{T}}^{\text {vis }}(S)$ and $|\mathfrak{A}(S)|$ are metric spaces and $\Gamma(S)$ acts on both by isometries. Moreover, the action on $|\mathfrak{A}(S)|$ is simplicial on the second baricentric subdivision, and so its orbits are discrete.

On the other hand, the map $\widehat{\boldsymbol{W}}^{\prime}$ is clearly proper, because $\widehat{\mathcal{T}}^{\mathrm{vis}}(S)(\underline{p}) / \Gamma(S)$ is compact for every $p \in \Delta^{n-1} \times[0, \infty)$. Hence, $\widehat{\boldsymbol{W}}^{\prime}$ is a homeomorphism. By Lemma C.1 b), $\widehat{\boldsymbol{W}}$ is a homeomorphism too.

\subsection{The bordification of arcs}

Define a map

$$
\Phi:|\mathfrak{A}(S)| \times[0, \infty] \rightarrow \overline{\mathcal{T}}^{a}(S)
$$

in the following way:

$$
\Phi(w,\|\underline{p}\|)= \begin{cases}\left(\left[\lambda_{\bullet}^{-1}\left(\widehat{\boldsymbol{W}}^{-1}(w, 0)\right)\right], 0\right) & \text { if }\|\underline{p}\|=0, \\ j\left(\widehat{\boldsymbol{W}}^{-1}(w,\|\underline{p}\|)\right) & \text { if } 0<\|\underline{p}\|<\infty \\ ([w], \infty) & \text { if }\|\underline{p}\|=\infty\end{cases}
$$

The situation is thus as in the following diagram:

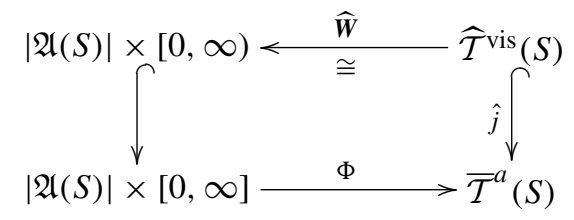

Theorem 3.17. $\Phi$ is a $\Gamma(S)$-equivariant homeomorphism. Thus, $\overline{\mathcal{M}}^{a}(S)=\overline{\mathcal{T}}^{a}(S) / \Gamma(S)$ is compact.

For homogeneity of notation, we set $\overline{\boldsymbol{W}}^{a}:=\Phi^{-1}: \overline{\mathcal{T}}^{a}(S) \rightarrow|\mathfrak{A}(S)| \times[0, \infty]$.

In order to prove Theorem 3.17, we need a few preliminary results.

Proposition 3.18. The map $\mathcal{T}(S) \hookrightarrow \overline{\mathcal{T}}^{a}(S)$ extends to a continuous $\hat{j}: \widehat{\mathcal{T}}^{\mathrm{vis}}(S) \hookrightarrow$ $\overline{\mathcal{T}}^{a}(S)$.

Proof. The continuity of $\hat{j}$ follows from Lemma B.2 Moreover, Lemmas 3.4 and 3.12 ensure that the $t$-lengths separate the points of $\widehat{\mathcal{T}}^{\mathrm{vis}}(S)$ and so $\hat{j}$ is injective.

Lemma 3.19. Let $\left\{\left[f_{m}: S \rightarrow \Sigma_{m}\right]\right\}$ be a sequence in $\mathcal{T}(S)$.

(a) $\left\|t_{\bullet}\left(f_{m}\right)\right\|_{\infty} \rightarrow 0$ if and only if $\left\|\mathcal{L}\left(f_{m}\right)\right\| \rightarrow 0$.

(b) $\left\|t_{\bullet}\left(f_{m}\right)\right\|_{\infty} \rightarrow \infty$ if and only if $\left\|\mathcal{L}\left(f_{m}\right)\right\| \rightarrow \infty$.

(c) $\left\|t_{\bullet}\left(f_{m}\right)\right\|_{\infty}$ is bounded away from zero $\Leftrightarrow\left\|\mathcal{L}\left(f_{m}\right)\right\|$ is bounded away from zero.

Proof. Because $w_{\mathrm{sp}}\left(\alpha, f_{m}\right) \leq t_{\alpha}\left(f_{m}\right)$ for $\alpha \in \boldsymbol{A}_{\mathrm{sp}}\left(f_{m}\right)$, we conclude

$$
(6 g-6+3 n)\left\|t_{\bullet}\left(f_{m}\right)\right\|_{\infty} \geq 2\left\|\mathcal{L}\left(f_{m}\right)\right\| .
$$

By the Collar Lemma A.1 $\ell_{\alpha}\left(f_{m}\right) \geq T\left(\left\|\mathcal{L}\left(f_{m}\right)\right\|\right) / 2$ for all $\alpha \in \mathcal{A}(S)$ and so $\left\|t_{\bullet}\left(f_{m}\right)\right\| \leq$ $T\left(T\left(\left\|\mathcal{L}\left(f_{m}\right)\right\|\right) / 2\right)$. 
Lemma 3.20. The map $\Phi$ is continuous and injective.

Proof. The injectivity of $\Phi$ is immediate.

As we already know that $\hat{j}$ is continuous, consider a sequence $\left\{\left[f_{m}: S \rightarrow \Sigma_{m}\right]\right\} \subset$ $\mathcal{T}(S)$ such that $\boldsymbol{W}\left(f_{m}\right) \rightarrow w \in|\mathfrak{A}(S)| \times\{\infty\}$, where $\boldsymbol{A}_{m}:=\operatorname{supp}\left(\boldsymbol{W}\left(f_{m}\right)\right)$ and $\boldsymbol{A}:=$ $\operatorname{supp}(w)=\left\{\alpha_{0}, \ldots, \alpha_{k}\right\}$.

We can assume that $\boldsymbol{A}_{m}$ is maximal, that $\boldsymbol{A} \subseteq \boldsymbol{A}_{m}$ and that $w\left(\alpha_{0}\right) \geq w\left(\alpha_{i}\right)$ for every $1 \leq i \leq k$

Convergence for the arcs in $\boldsymbol{A}$. For every $\alpha_{i} \in \boldsymbol{A}$, equation $*$ of Remark 3.10 applied to $\boldsymbol{A}_{m}$ gives $\ell_{\alpha_{i}}\left(f_{m}\right) \rightarrow 0$ and so

$$
\cos \left(\gamma\left(\vec{\alpha}_{i}, f_{m}\right)\right) \geq 1-s_{\alpha_{i}}^{2} / 2 \geq 1 / 2-\varepsilon_{m}
$$

for large $m$. This implies that

$$
w_{\mathrm{sp}}\left(\alpha_{i}, f_{m}\right) \approx 2 \log \left(4 \cos \left(\gamma\left(\vec{\alpha}_{i}, f_{m}\right)\right)\right)+2 \log \left(4 \cos \left(\gamma\left(\overleftarrow{\alpha}_{i}, f_{m}\right)\right)\right)-4 \log \left(\ell_{\alpha_{i}}\left(f_{m}\right)\right)
$$

and so

$$
\frac{t_{\alpha_{i}}\left(f_{m}\right)}{t_{\alpha_{0}}\left(f_{m}\right)} \approx \frac{\log \left(\ell_{\alpha_{i}}\left(f_{m}\right)\right)}{\log \left(\ell_{\alpha_{0}}\left(f_{m}\right)\right)} \approx \frac{w_{\mathrm{sp}}\left(\alpha_{i}, f_{m}\right)}{w_{\mathrm{sp}}\left(\alpha_{0}, f_{m}\right)} \rightarrow \frac{w\left(\alpha_{i}\right)}{w\left(\alpha_{0}\right)} .
$$

Uniform convergence for the arcs not in $A$. To conclude that proof, we need to show that for all $\beta \notin \boldsymbol{A}, t_{\beta}\left(f_{m}\right) / t_{\alpha_{0}}\left(f_{m}\right) \rightarrow 0$ uniformly.

For contradiction, up to subsequences, we can suppose that there are $\eta>0$ and $\left\{\beta_{m}\right\} \subset \mathcal{A}(S) \backslash \boldsymbol{A}$ such that $t_{\beta_{m}}\left(f_{m}\right) / t_{\alpha_{0}}\left(f_{m}\right) \geq \eta$ and so $\ell_{\beta_{m}}\left(f_{m}\right) \rightarrow 0$. As a consequence, as $m$ becomes large, $\beta_{m}$ cannot intersect $\boldsymbol{A}_{m}$ and so $\beta_{m} \in \boldsymbol{A}_{m}$. In fact, if $\beta_{m} \cap \alpha^{\prime} \neq \varnothing$ with $\alpha^{\prime} \in \boldsymbol{A}_{m}$, then $\alpha^{\prime}$ would be much longer than $\beta_{m}$ by the Collar Lemma A.1 Hence, the shortest path from a point in $\beta_{m} \cap \alpha^{\prime}$ to the boundary would follow $\beta_{m}$ rather than $\alpha^{\prime}$, and so $\alpha^{\prime}$ would not belong to $\boldsymbol{A}_{m}$. Then, by equation $(*)$,

$$
\sinh \left(w_{\mathrm{sp}}\left(\vec{\beta}_{m}, f_{m}\right)\right)=\frac{s_{x}^{2}+s_{y}^{2}-s_{\beta_{m}}^{2}}{2 s_{x} s_{y} \sqrt{s_{\beta_{m}}^{2}-1}} \approx \frac{s_{x}^{2}+s_{y}^{2}-1}{s_{x} s_{y} \ell_{\beta_{m}}} \geq \frac{1}{\ell_{\beta_{m}}} .
$$

Thus, asymptotically $w_{\mathrm{sp}}\left(\beta_{m}, f_{m}\right) \geq 4 \log \left(2 / \ell_{\beta_{m}}\left(f_{m}\right)\right) \approx 2 t_{\beta_{m}}\left(f_{m}\right)$. As $w_{\mathrm{sp}}\left(\alpha_{0}, f_{m}\right) \approx$ $2 t_{\alpha_{0}}\left(f_{m}\right)$, we conclude that $w_{\mathrm{sp}}\left(\beta_{m}, f_{m}\right) / w_{\mathrm{sp}}\left(\alpha_{0}, f_{m}\right) \geq \eta / 2$ for $m$ large. But

$$
\sup _{\beta \in \boldsymbol{A}_{m} \backslash \boldsymbol{A}} \frac{w_{\mathrm{sp}}\left(\beta, f_{m}\right)}{w_{\mathrm{sp}}\left(\alpha_{0}, f_{m}\right)} \rightarrow 0 .
$$

This contradiction proves the claim.

Proposition 3.21. $\Gamma(S)$ acts on $\overline{\mathcal{T}}^{a}(S)$ by isometries and with discrete orbits. Hence, $\overline{\mathcal{M}}^{a}(S)=\overline{\mathcal{T}}^{a}(S) / \Gamma(S)$ is Hausdorff.

Proof. Suppose $t \cdot g_{m} \rightarrow t$ with $t \in \overline{\mathcal{T}}^{a}(S)$ and $g_{m} \in \Gamma(S)$. Consider a sequence $\left\{\left[f_{m}: S \rightarrow \Sigma_{m}\right]\right\}$ such that $j\left(f_{m}\right) \rightarrow t$ in $\overline{\mathcal{T}}^{a}(S)$. 
Case $\|t\|_{\infty}>0$ finite. Passing to a subsequence, $\left[f_{m}\right] \cdot h_{m} \rightarrow[f: S \rightarrow \Sigma] \in$ $\widehat{\mathcal{T}}^{\text {vis }}(S)$ for suitable $h_{m} \in \Gamma(S)$. Thus, $\hat{j}(f) \cdot h_{m}^{-1} \rightarrow t$.

Let $\boldsymbol{A}=f^{-1}\left(\boldsymbol{A}_{\mathrm{sp}}(\Sigma)\right)$, so that it is supported on $f^{-1}\left(\Sigma_{+}\right)$and $\ell_{\alpha}(f)<\infty$ for all $\alpha \in \boldsymbol{A}$. Because the length spectrum of finite arcs in $\Sigma$ is discrete (with finite multiplicities) and $\hat{j}(f) \cdot h_{m}^{-1}$ is a Cauchy sequence, $h_{m}^{-1}$ fixes $\boldsymbol{A}$ for $m$ large (up to subsequences). Thus, we can assume that $h_{m}$ is a diffeomorphism that restricts to an isometry on $f^{-1}\left(\Sigma_{+}\right)$(with the pull-back metric). Hence, $t=\hat{j}(f) \cdot h_{m}^{-1}$ for $m$ large and so $t=\hat{j}(\hat{f})$ for some $\hat{f}: S \rightarrow \Sigma$. Similarly, $\hat{j}(\hat{f}) \cdot g_{m} \rightarrow \hat{j}(\hat{f})$ and so $g_{m}$ is a diffeomorphism that restricts to an isometry on $\hat{f}^{-1}\left(\Sigma_{+}\right)$for large $m$. Hence, $t \cdot g_{m}$ cannot accumulate at $t$.

Case $\|t\|_{\infty}=0$. The surface has $n$ cusps. It follows from the classical case that the spectrum of the finite reduced lengths (and so of the finite $\lambda$-lengths) of $(\Sigma, p)$ is discrete and with finite multiplicities. Because $\left[t_{\bullet}(f)\right]=\left[\lambda_{\bullet}(f)\right]$, we can conclude as in the previous case.

Case $\|t\|_{\infty}=\infty$. Let $w^{(m)}=\boldsymbol{W}\left(f_{m}\right)$. Up to subsequences, $w^{(m)} \cdot h_{m} \rightarrow w$ in $|\mathfrak{A}(S)| \times[0, \infty]$ for suitable $h_{m} \in \Gamma(S)$ and $w \in|\mathfrak{A}(S)| \times\{\infty\}$. As before, $\Phi(w) \cdot h_{m}^{-1} \rightarrow t$ in $\overline{\mathcal{T}}^{a}(S)$. Because $w$ has finite support, $t=\Phi(w) \cdot h_{m}^{-1}$ for $m$ large, and so $t=\Phi(\hat{w})$ for some $\hat{w} \in|\mathfrak{A}(S)| \times\{\infty\}$. Thus, $\Phi(\hat{w}) \cdot g_{m} \rightarrow \Phi(\hat{w})$ and $g_{m}$ is a diffeomorphism that restricts to an isometry on $S_{+}$, where $S_{+}$is the $\hat{w}$-visible subsurface of $S$. Hence, $t \cdot g_{m}$ cannot accumulate at $t$.

Proof of Theorem 3.17. In order to apply Lemma C.1 (b), we only need to prove that $\Phi^{\prime}:|\mathfrak{A}(S)| / \Gamma(S) \times[0, \infty] \rightarrow \overline{\mathcal{M}}^{a}(S)$ is a homeomorphism.

We already know that $\Phi^{\prime}$ is continuous, injective. Moreover, its image contains $\mathcal{M}(S)$, which is dense in $\overline{\mathcal{M}}^{a}(S)$. As $|\mathfrak{A}(S)| / \Gamma(S)$ is compact and $\overline{\mathcal{M}}^{a}(S)$ is Hausdorff, the map $\Phi^{\prime}$ is closed and so it is also surjective. Hence, $\Phi^{\prime}$ is a homeomorphism.

Corollary 3.22. $\hat{j}$ is a homeomorphism onto the finite part of $\overline{\mathcal{T}}^{a}(S)$.

\subsection{The extended Teichmüller space}

We define the extended Teichmüller space $\tilde{\mathcal{T}}(S)$ to be

$$
\widetilde{\mathcal{T}}(S):=\overline{\mathcal{T}}(S) \cup|\mathfrak{A}(S)|_{\infty}
$$

where $|\mathfrak{A}(S)|_{\infty}$ is just a copy of $|\mathfrak{A}(S)|$.

Clearly, there is a map $\mathrm{Bl}_{0} \tilde{\mathcal{T}}(S) \rightarrow \overline{\mathcal{T}}^{a}(S)$, which identifies visibly equivalent surfaces of $\widehat{\mathcal{T}}(S) \subset \mathrm{Bl}_{0} \tilde{\mathcal{T}}(S)$.

We define a topology on $\tilde{\mathcal{T}}(S)$ by requiring that $\overline{\mathcal{T}}(S) \hookrightarrow \widetilde{\mathcal{T}}(S)$ and $|\mathfrak{A}(S)|_{\infty} \hookrightarrow$ $\tilde{\mathcal{T}}(S)$ are homeomorphisms onto their images, that $\overline{\mathcal{T}}(S) \subset \tilde{\mathcal{T}}(S)$ is open and we declare that a sequence $\left\{f_{m}\right\} \subset \overline{\mathcal{T}}(S)$ is converging to $w \in|\mathfrak{A}(S)|_{\infty}$ if and only if $\boldsymbol{W}\left(f_{m}\right) \rightarrow$ $(w, \infty)$ in $|\mathfrak{A}(S)| \times(0, \infty]$.

Notice that $\widetilde{\mathcal{M}}(S):=\tilde{\mathcal{T}}(S) / \Gamma(S)$ is an orbifold with corners, which acquires some singularities at infinity. In fact, $\widetilde{\mathcal{M}}(S)$ is homeomorphic to $\overline{\mathcal{M}}(R, x) \times \Delta^{n-1} \times[0, \infty] / \sim$, where $(R, x)$ is a closed $x$-marked surface such that $S \simeq R \backslash x$ and $\left(R^{\prime}, \underline{p}^{\prime}, t^{\prime}\right) \sim$ $\left(R^{\prime \prime}, \underline{p}^{\prime \prime}, t^{\prime \prime}\right) \Leftrightarrow t^{\prime}=t^{\prime \prime}=\infty$ and $\left(R^{\prime}, \underline{p}^{\prime}\right)$ is visibly equivalent to $\left(R^{\prime \prime}, \underline{p}^{\prime \prime}\right)$. 


\section{Weil-Petersson form and circle actions}

\subsection{Circle actions on moduli spaces}

Now we extend some of the previous constructions to the case of surfaces with marked points on the boundary.

Let $S$ be a compact surface of genus $g$ with boundary components $C_{1}, \ldots, C_{n}$ (assume as usual that $2 g-2+n>0$ ). Moreover, let $v_{i}$ be a point of $C_{i}$ and set $v=$ $\left(v_{1}, \ldots, v_{n}\right)$.

We stress that the role of $v$ is different than that of the usual marked points $x$, as the metrics we consider are smooth at $v$. In fact, $v_{i}$ must be understood as a sort of twist parameter at $C_{i}$.

In this new situation, $\operatorname{Diff}_{+}(S, v)$ will be the group of orientation-preserving diffeomorphisms of $S$ that fix $v$ pointwise, $\mathcal{T}(S, v)$ will be the space of hyperbolic metrics on $S$ up to the action of $\operatorname{Diff}_{0}(S, v)$, and $\Gamma(S, v)=\operatorname{Diff}_{+}(S, v) / \operatorname{Diff}_{0}(S, v)$. Thus, $\mathcal{M}(S, v)=\mathcal{T}(S, v) / \Gamma(S, v)$ is the resulting moduli space.

Clearly, $\mathbb{R}^{n}$ acts on $\mathcal{T}(S, v)$ by Fenchel-Nielsen twist (with unit angular speed) around the boundary components and $\mathcal{T}(S, v) / \mathbb{R}^{n}=\mathcal{T}(S)$. Similarly, the torus $\mathbb{T}^{n}=$ $(\mathbb{R} / 2 \pi \mathbb{Z})^{n}$ acts on $\mathcal{M}(S, v)$ and the quotient is $\mathcal{M}(S, v) / \mathbb{T}^{n}=\mathcal{M}(S)$.

Again we can define an augmented Teichmüller space $\overline{\mathcal{T}}(S, v)$ and an action of $\mathbb{R}^{n}$ on it. However, we want to be a little more careful and require that a marking $[f: S \rightarrow$ $\Sigma] \in \overline{\mathcal{T}}(S, v)$ that shrinks $C_{i}$ to a cusp $y_{i} \in \Sigma$ is smooth with $\operatorname{rk}(d f)=1$ at $C_{i}$, so that $f$ identifies $C_{i}$ with the sphere tangent bundle $S T_{\Sigma, y_{i}}$, and $v_{i}$ with a point in $S T_{\Sigma, y_{i}}$.

Thus, $\overline{\mathcal{T}}(S, v) \rightarrow \overline{\mathcal{T}}(S)$ is an $\mathbb{R}^{n}$-bundle and $\overline{\mathcal{M}}(S, v) \rightarrow \overline{\mathcal{M}}(S)$ is a $\mathbb{T}^{n}$-bundle, which is a product $L_{1} \times \cdots \times L_{n}$ of circle bundles $L_{i}$ associated to $v_{i} \in C_{i}$.

If one wishes, one can certainly lift the action to $\widehat{\mathcal{T}}(S, v)=\mathrm{Bl}_{0} \overline{\mathcal{T}}(S, v)$.

We also write $\widehat{\mathcal{T}}^{\text {vis }}(S, x):=\widehat{\mathcal{T}}(S, x) / \sim_{\text {vis }}$, where the visible equivalence on $\widehat{\mathcal{T}}(S, x)$ is defined as in Section 3.4 .

This means that if $[f: S \rightarrow \Sigma] \in \widehat{\mathcal{T}}^{\mathrm{vis}}(S, v)$ has $f\left(C_{i}\right) \nsubseteq \bar{\Sigma}_{+}$, then $[f]$ does not record the exact position of the point $v_{i} \in C_{i}$. In other words, the $i$-th component of $\mathbb{R}^{n}$ acts trivially on $[f]$.

\subsection{The arc complex of $(S, v)$}

Denote by $\mathcal{A}(S, v)$ the set of nontrivial isotopy classes of simple arcs in $S$ that start and end at $\partial S \backslash v$ and let $\beta_{i}$ be a (fixed) arc from $C_{i}$ to $C_{i}$ that separates $v_{i}$ from the rest of the surface.

A subset $\boldsymbol{A}=\left\{\beta_{1}, \ldots, \beta_{n}, \alpha_{1}, \ldots, \alpha_{k}\right\} \subset \mathcal{A}(S, v)$ is a $k$-system of $\operatorname{arcs}$ on $(S, v)$ if $\beta_{1}, \ldots, \beta_{n}, \alpha_{1}, \ldots, \alpha_{k}$ are homotopically nontrivial, pairwise nonhomotopic and they admit disjoint representatives. A point in the arc complex $\mathfrak{A}(S, v)$ can be represented as a sum $\sum_{j} w_{j} \alpha_{j}$, provided we remember the $\beta_{i}$ 's (that is, as $\sum_{j} w_{j} \alpha_{j}+\sum_{i} 0 \beta_{i}$ ).

As before, $\mathfrak{A}^{\circ}(S, v) \subset \mathfrak{A}(S, v)$ is made of systems of arcs that cut $S$ into a disjoint union of discs and annuli homotopic to some boundary component.

Remark that there is a natural map $\mathfrak{A}(S, v) \rightarrow \mathfrak{A}(S)$, induced by the inclusion $S \backslash v \hookrightarrow S$ and that forgets the $\beta_{i}$ 's, and so a simplicial map $|\mathfrak{A}(S, v)| \rightarrow|\mathfrak{A}(S)|$. 
We can also define a suitable map $\widehat{\boldsymbol{W}}_{v}$ for the pointed surface $(S, v)$ in such a way that the following diagram commutes:

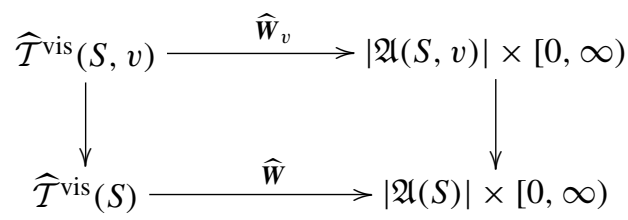

Let $[f: S \rightarrow \Sigma] \in \widehat{\mathcal{T}}^{\mathrm{vis}}(S, v)$. If we consider it as a point of $\widehat{\mathcal{T}}^{\mathrm{vis}}(S)$, then $\widehat{\boldsymbol{W}}(f)$ is a system of $\operatorname{arcs}$ in $S$.

For every $i=1, \ldots, n$ such that $f\left(C_{i}\right) \in \Sigma_{+}$, consider the geodesic $\rho_{i} \subset \Sigma$ coming out from $f\left(v_{i}\right)$ and perpendicular to $f\left(C_{i}\right)$ (if $f\left(C_{i}\right)$ is a cusp, let $\rho_{i}$ be the geodesic originating at $f\left(C_{i}\right)$ in direction $\left.f\left(v_{i}\right)\right)$. Let $z_{i}$ be the point where $\rho_{i}$ first meets the spine of $\Sigma$, and $e_{i}$ an infinitesimal portion of $\rho_{i}$ starting at $z_{i}$ and going towards $f\left(C_{i}\right)$.

Define $\operatorname{Sp}(\Sigma, f(v))$ to be the one-dimensional CW-complex obtained from $\operatorname{Sp}(\Sigma)$ by adding the vertices $z_{i}$ (in case $z_{i}$ was not already a vertex) and the infinitesimal edges $e_{i}$. Consequently, we have a well-defined system of $\operatorname{arcs} \boldsymbol{A}_{\mathrm{sp}}(\Sigma, f(v))$ dual to $\operatorname{Sp}(\Sigma, f(v))$ and widths $w_{\mathrm{sp}, \mathrm{f}(\mathrm{v})}$, in which the arc dual to $e_{i}$ plays the role of $f\left(\beta_{i}\right)$ (which thus has zero weight).

We set $\widehat{\boldsymbol{W}}_{v}(f)=f^{*} w_{\mathrm{sp}, \mathrm{f}(\mathrm{v})}$.

The following is an immediate consequence of Theorem 3.16 .

Proposition 4.1. The map $\widehat{\boldsymbol{W}}_{v}$ is a $\Gamma(S, v)$-equivariant homeomorphism.

We can make $\mathbb{R}^{n}$ act on $|\mathfrak{A}(S, v)|$ via $\widehat{\boldsymbol{W}}_{v}$ and so on $|\mathfrak{A}(S, v)| \times[0, \infty]$. Thus, the action also prolongs to the extended Teichmüller space $\widetilde{\mathcal{T}}(S, v):=\overline{\mathcal{T}}(S, v) \cup|\mathfrak{A}(S, v)|_{\infty}$.

\subsection{Weil-Petersson form}

If we choose a maximal set of simple closed curves $\gamma=\left\{\gamma_{1}, \ldots, \gamma_{6 g-6+2 n}, C_{1}, \ldots, C_{n}\right\}$ on $S$, we can define a symplectic form $\omega_{v}$ on $\mathcal{T}(S, v)$ by setting

$$
\omega_{v}=\sum_{i=1}^{6 g-6+2 n} d \ell_{i} \wedge d \tau_{i}+\sum_{j=1}^{n} d p_{j} \wedge d t_{j}=\sum_{j=1}^{n} d\left(p_{j}^{2} / 2\right) \wedge d\left(\vartheta_{j} / 2 \pi\right)
$$

where $t_{j}=p_{j} \vartheta_{j} / 2 \pi$ is the twist parameter at $C_{j}$. As usual, $\omega_{v}$ does not depend on the choice of $\gamma$ and it descends to $\mathcal{M}(S, v)$. Its independence from the particular FenchelNielsen coordinates permits us to extend $\omega_{v}$ to a symplectic form on $\overline{\mathcal{M}}(S, v)$.

Moreover, the twist flow on $\overline{\mathcal{M}}(S, v)$ is Hamiltonian and the associated moment map is exactly $\mu=\left(p_{1}^{2} / 2, \ldots, p_{n}^{2} / 2\right)$. Thus, the leaves $\left(\overline{\mathcal{M}}(S)(\underline{p}), \omega_{\underline{p}}\right)$ are exactly the symplectic reductions of $\left(\overline{\mathcal{M}}(S, v), \omega_{v}\right)$ with respect to the $\mathbb{T}^{n}$-action.

As remarked by Mirzakhani [Mir07], it follows by standards results of symplectic geometry that there is a symplectomorphism $\overline{\mathcal{M}}(S)(\underline{p}) \rightarrow \overline{\mathcal{M}}(S)(0)$ which pulls $\left[\omega_{0}\right]+$ $\sum_{i=1}^{n}\left(p_{i}^{2} / 2\right) c_{1}\left(L_{i}\right)$ back to $\left[\omega_{\underline{p}}\right]$. 
Penner has provided a beautiful formula for $\omega_{0}$ in terms of the $\tilde{a}$-coordinates (see Appendix $B$ for the precise definitions).

Theorem 4.2 ([ए户en92]). Let $\boldsymbol{A}$ be a maximal system of arcs on $S$. If $\pi: \mathcal{T}(S)(0) \times \mathbb{R}_{+}^{n}$ $\rightarrow \mathcal{T}(S)(0)$ is the projection onto the first factor, then

$$
\pi^{*} \omega_{0}=-\frac{1}{2} \sum_{H}\left(d \tilde{a}_{1}^{H} \wedge d \tilde{a}_{2}^{H}+d \tilde{a}_{2}^{H} \wedge d \tilde{a}_{3}^{H}+d \tilde{a}_{3}^{H} \wedge d \tilde{a}_{1}^{H}\right)
$$

where $H$ ranges over all the ideal triangles in $S \backslash \boldsymbol{A}$ and it is bounded by the (cyclically ordered) arcs $\left(\alpha_{1}^{H}, \alpha_{2}^{H}, \alpha_{3}^{H}\right)$, and $\tilde{a}_{i}^{H}$ is the reduced length of $\alpha_{i}^{H}$.

The whole $\mathcal{T}(S)$ is naturally a Poisson manifold with the Weil-Petersson pairing $\eta$ on the cotangent bundle, whose symplectic leaves are the $\mathcal{T}(S)(p)$. A general formula expressing $\eta$ in terms of lengths of arcs and widths is given by the following.

Theorem 4.3 ([Mon09c] $)$. Let $\boldsymbol{A}$ be a maximal system of arcs on $S$. Then

$$
\eta=\frac{1}{4} \sum_{k=1}^{n} \sum_{\substack{y_{i} \in \alpha_{i} \cap C_{k} \\ y_{j} \in \alpha_{j} \cap C_{k}}} \frac{\sinh \left(p_{k} / 2-d_{C_{k}}\left(y_{i}, y_{j}\right)\right)}{\sinh \left(p_{k} / 2\right)} \frac{\partial}{\partial a_{i}} \wedge \frac{\partial}{\partial a_{j}}
$$

where $d_{C_{k}}\left(y_{i}, y_{j}\right)$ is the length of the geodesic running from $y_{i}$ to $y_{j}$ along $C_{k}$ in the positive direction.

In order to understand the limit for large $p$, it makes sense to rescale the main quantities as $\tilde{w}_{i}=(\|\mathcal{L}\| / 2)^{-1} w_{i}, \tilde{\omega}=(1+\|\mathcal{L}\| / 2)^{-2} \omega$ and $\tilde{\eta}=(1+\|\mathcal{L}\| / 2)^{2} \eta$.

Lemma 4.4 ([|Kon92]). The class $\left[\tilde{\omega}_{\infty}\right] \in H_{\Gamma(S)}^{2}(|\mathfrak{A}(S)|)$ is represented by a piecewise linear 2-form on $|\mathfrak{A}(S)|$ whose dual can be written (on the maximal simplices) as

$$
\tilde{H}=\frac{1}{2} \sum_{V}\left(\frac{\partial}{\partial \tilde{w}_{1}^{V}} \wedge \frac{\partial}{\partial \tilde{w}_{2}^{V}}+\frac{\partial}{\partial \tilde{w}_{2}^{V}} \wedge \frac{\partial}{\partial \tilde{w}_{3}^{V}}+\frac{\partial}{\partial \tilde{w}_{3}^{V}} \wedge \frac{\partial}{\partial \tilde{w}_{1}^{V}}\right)
$$

where $V$ ranges over all the trivalent vertices of the ribbon graph represented by a point in $\left|\mathfrak{A}^{\circ}(S)\right|,\left(E_{1}^{V}, E_{2}^{V}, E_{3}^{V}\right)$ is the cyclically ordered triple of edges incident at $V$, and $\tilde{w}_{i}^{V}$ is the normalized width of $E_{i}$ (see Appendix $B$ ).

The above result admits a pointwise sharpening as follows.

Theorem 4.5 ([Mon09c] $)$. The bivector field $\tilde{\eta}$ extends over $\tilde{\mathcal{T}}(S)$ and, on the maximal simplices of $|\mathfrak{A}(S)|_{\infty}$, we have

$$
\tilde{\eta}_{\infty}=\tilde{H} \quad \text { pointwise }
$$

Thus, we have a description of the degeneration of $\eta$ when the boundary lengths of the hyperbolic surface become very large. 


\section{From surfaces with boundary to pointed surfaces}

\subsection{Ribbon graphs}

Let $S$ be a compact oriented surface of genus $g$ with boundary components $C_{1}, \ldots, C_{n}$ and assume that $\chi(S)=2-2 g-n<0$. Let $\boldsymbol{A}=\left\{\alpha_{0}, \ldots, \alpha_{k}\right\} \in \mathfrak{A}(S)$ be a system of $\operatorname{arcs}$ in $S$ with associated visible subsurface $S_{+}$.

If $\vec{\alpha}$ is an oriented arc supported on $\alpha$, then we will refer to $\vec{\alpha}^{*}$ as the oriented edge dual to $\vec{\alpha}$.

Remark 5.1. If $S$ carries a hyperbolic metric and $\boldsymbol{A}$ is its spinal system of arcs, then $\vec{\alpha}^{*}$ must be considered the edge of the spine dual to $\alpha$ and oriented in such a way that, at the point $\vec{\alpha}^{*} \cap \vec{\alpha}$ (unique, up to prolonging $\vec{\alpha}^{*}$ ), the tangent vectors $\left\langle v_{\vec{\alpha}^{*}}, v_{\vec{\alpha}}\right\rangle$ form a positive basis of $T_{p} S$.

Let $E(\boldsymbol{A}):=\left\{\vec{\alpha}^{*}, \bar{\alpha}^{*} \mid \alpha \in \boldsymbol{A}\right\}$ and define the following operators $\sigma_{0}, \sigma_{1}, \sigma_{\infty}$ on $E(\boldsymbol{A})$ :

(1) $\sigma_{1}$ reverses the orientation of each arc (i.e. $\sigma_{1}\left(\vec{\alpha}^{*}\right)=\bar{\alpha}^{*}$ ),

$(\infty)$ if $\vec{\alpha}$ ends at $x_{\alpha} \in C_{i}$, then $\sigma_{\infty}\left(\vec{\alpha}^{*}\right)$ is dual to the oriented $\operatorname{arc} \vec{\beta}$ that ends at $x_{\beta} \in C_{i}$, where $x_{\beta}$ comes just before $x_{\alpha}$ according to the orientation induced on $C_{i}$ by $S$,

(0) $\sigma_{0}$ is defined by $\sigma_{0}=\sigma_{1} \sigma_{\infty}^{-1}$.

If we let $E_{i}(\boldsymbol{A})$ denote the orbits of $E(\boldsymbol{A})$ under the action of $\sigma_{i}$, then

(1) $E_{1}(\boldsymbol{A})$ can be identified with $\boldsymbol{A}$,

$(\infty) E_{\infty}(\boldsymbol{A})$ can be identified with the subset of the boundary components of $S$ that belong to $S_{+}$,

(0) $E_{0}(\boldsymbol{A})$ can be identified with the set of connected components of $S_{+} \backslash \boldsymbol{A}$.

\subsection{Flat tiles and Jenkins-Strebel differentials}

Keeping the notation as before, let $f: S \rightarrow \hat{S}$ be the topological type of $\boldsymbol{A}$ (see Section 3.4.

For every system of weights $w$ supported on $\boldsymbol{A}$, the surface $\hat{S}_{+}$can be endowed with a flat metric (with conical singularities) in the following way.

Every component $\hat{S}_{i,+}$ of $\hat{S}_{+}$is quasi-filled by the arc system $f(\boldsymbol{A}) \cap \hat{S}_{i,+}$. As we can carry on the construction componentwise, we can assume that $\boldsymbol{A}$ quasi-fills $S$.

In this case, we consider the flat tile $F T=[0,1] \times[0, \infty] /[0,1] \times\{\infty\}$ and we call the class $[0,1] \times\{\infty\}$ the point at infinity. Moreover, we define $\Sigma:=\bigcup_{\vec{e} \in E(\boldsymbol{A})} F T_{\vec{e}} / \sim$, where $F T_{\vec{e}}:=F T \times\{\vec{e}\}$ and

- $(u, 0, \vec{e}) \sim(1-u, 0, \grave{e})$ for all $\vec{e} \in E(\boldsymbol{A})$ and $u \in[0,1]$,

- $(1, v, \vec{e}) \sim\left(0, v, \sigma_{\infty}(\vec{e})\right)$ for all $\vec{e} \in E(\boldsymbol{A})$ and $v \in[0, \infty]$.

We can also define an embedded graph $G \subset \Sigma$ by gluing the segments $[0,1] \times\{0\} \subset F T$ contained in each tile. Thus, we can identify $\alpha^{*}$ with an unoriented edge of $G$ for every $\alpha \in \boldsymbol{A}$.

It is easy to check that there is a homeomorphism $\hat{S} \rightarrow \Sigma$, well-defined up to isotopy, that takes boundary components to points at infinity or to vertices. 


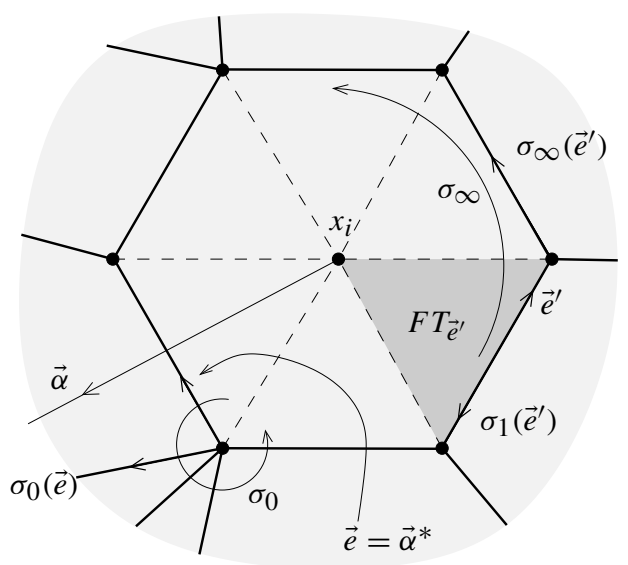

Fig. 3. Local structure of a flat surface quasi-filled by $\boldsymbol{A}$.

Moreover, for every $\vec{\alpha}^{*} \in E(\boldsymbol{A})$, we can endow $F T_{\vec{\alpha}^{*}}$ with the quadratic differential $d z^{2}$, where $z=w(\alpha) u+i v$. These quadratic differentials glue to give a global $\varphi$ (and so a conformal structure on the whole $\Sigma$ ), which has double poles with negative quadratic residues at the points at infinity and is holomorphic elsewhere, with zeroes of order $k-2$ at the $k$-valent vertices of $G$. Furthermore, $\alpha^{*}$ has length $w(\alpha)$ with respect to the induced flat metric $|\varphi|$.

Finally, the horizontal trajectories of $\varphi$ (that is, the curves along which $\varphi$ is positivedefinite) are either closed circles that wind around some point at infinity, or edges of $G$.

Thus, $\varphi$ is a Jenkins-Strebel quadratic differential and $G$ is its critical graph, i.e. the union of all horizontal trajectories that hit some zero or some pole of $\varphi$.

If $\boldsymbol{A}$ does not quasi-fill $S$, then we will define the Jenkins-Strebel differential componentwise, by setting it to zero on the invisible components.

See [Har86], [Kon92], [Loo95] and [Mon09b] for more details.

\subsection{HMT construction}

We begin by recalling the following result of Strebel.

Theorem 5.2 ([Str67]). Let $R^{\prime}$ be a compact Riemann surface of genus $g$ with $x^{\prime}=$ $\left(x_{1}^{\prime}, \ldots, x_{n}^{\prime}\right)$ distinct points such that $n \geq 1$ and $2 g-2+n>0$. For every $\left(p_{1}, \ldots, p_{n}\right) \in$ $\mathbb{R}_{\geq 0}^{n}$ (but not all zero), there exists a unique (nonzero) quadratic differential $\varphi$ on $R^{\prime}$ such that

- $\varphi$ is holomorphic on $R^{\prime} \backslash x^{\prime}$,

- horizontal trajectories of $\varphi$ are either circles that wind around some $x_{i}^{\prime}$ or closed arcs between critical points,

- the critical graph $G$ of $\varphi$ cuts $R^{\prime}$ into semi-infinite flat cylinders (according to the metric $|\varphi|)$, whose circumferences are closed trajectories, 
- if $p_{i}=0$, then $x_{i}^{\prime}$ belongs to the critical graph,

- if $p_{i}>0$, then the cylinder around $x_{i}^{\prime}$ has circumference length $p_{i}$.
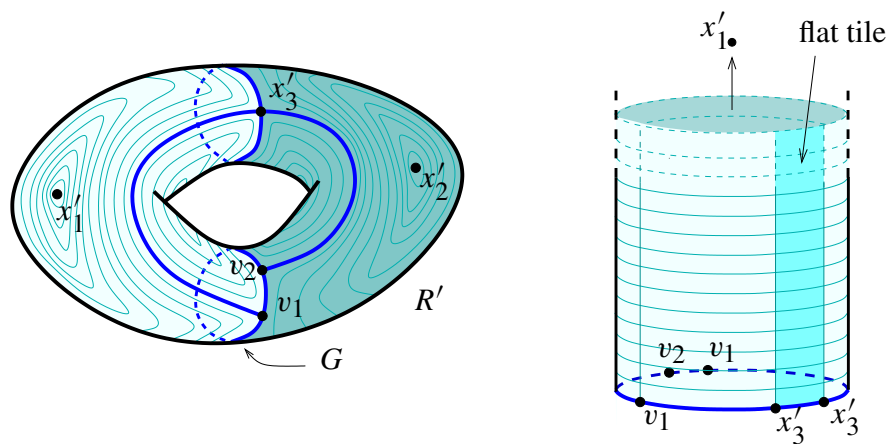

Fig. 4. An example of Jenkins-Strebel differential and its horizontal foliation with $p_{1}, p_{2}>0$ and $p_{3}=0$.

Notice that the graph $G$ plays a role analogous to the spine of a hyperbolic surface. In fact, given a point $\left[f: R \rightarrow R^{\prime}\right] \in \mathcal{T}(R, x)$ and $\left(p_{1}, \ldots, p_{n}\right) \in \Delta^{n-1}$, we can consider the unique $\varphi$ given by the theorem above and the system of $\operatorname{arcs} \boldsymbol{A} \in \mathfrak{A}^{\circ}(R, x)$ such that $f(\boldsymbol{A})$ is dual to the critical graph $G$ of $\varphi$, and we can define the width $w(\alpha)$ to be the $|\varphi|$-length of the edge $\alpha^{*}$ of $G$ dual to $\alpha \in A$.

Theorem 5.3 (Harer-Mumford-Thurston [Har86]). The map $\mathcal{T}(R, x) \times \Delta^{n-1} \rightarrow$ $\left|\mathfrak{A}^{\circ}(R, x)\right|$ just constructed is a $\Gamma(R, x)$-equivariant homeomorphism.

Clearly, if $R^{\prime}$ is a stable Riemann surface, then the theorem can be applied on every visible component of $R^{\prime}$ (i.e. on every component that contains some $x_{i}^{\prime}$ with $p_{i}>0$ ) and $\varphi$ can be extended by zero on the remaining part of $R^{\prime}$. Hence, we can extend the previous map to

$$
\boldsymbol{W}_{\mathrm{HMT}}: \overline{\mathcal{T}}^{\mathrm{vis}}(R, x) \times \Delta^{n-1} \rightarrow|\mathfrak{A}(R, x)|
$$

which is also a $\Gamma(R, x)$-equivariant homeomorphism (see, for instance, [Loo95] and [Mon09b]).

The purpose of the following sections is to relate this $\boldsymbol{W}_{\text {HMT }}$ to the spine construction.

\subsection{The grafting map}

Given a hyperbolic surface $\Sigma$ with boundary components $C_{1}, \ldots, C_{n}$, we can graft a semi-infinite flat cylinder at each $C_{i}$ of circumference $p_{i}=\ell\left(C_{i}\right)$. The result is a surface $\operatorname{gr}_{\infty}(\Sigma)$ with a $C^{1,1}$-metric (see Figure 5, called the Thurston metric (see [Tan97] and [SW02] for the case of a general lamination, or [KP94] for higher dimensional analogues). If $\Sigma$ has cusps, we do not glue any cylinder at the cusps of $\Sigma$. Notice that $\operatorname{gr}_{\infty}(\Sigma)$ has the conformal type of a punctured Riemann surface and it will sometimes be regarded as a closed Riemann surface with marked points. 


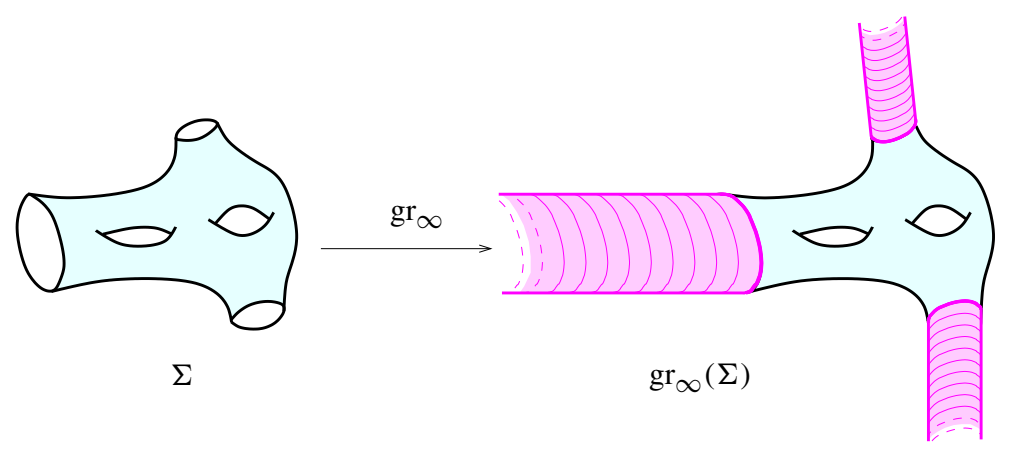

Fig. 5. An example of a surface obtained by infinite grafting.

Notation. Choose a closed surface $R$ with distinct marked points $x=\left(x_{1}, \ldots, x_{n}\right) \subset R$ and an identification $R \backslash x \cong \operatorname{gr}_{\infty}(S)$ such that $x_{i}$ corresponds to $C_{i}$. Clearly, we can identify $\mathfrak{A}(S) \cong \mathfrak{A}(R, x)$ and $\Gamma(S) \cong \Gamma(R, x)$.

We use the grafting construction to define a map

$$
\left(\mathrm{gr}_{\infty}, \mathcal{L}\right): \tilde{\mathcal{T}}(S) \rightarrow \overline{\mathcal{T}}(R, x) \times \Delta^{n-1} \times[0, \infty] / \sim
$$

where $\sim$ identifies $\left(\left[f_{1}\right], \underline{p}, \infty\right)$ and $\left(\left[f_{2}\right], \underline{p}, \infty\right)$ if $\left(\left[f_{1}\right], \underline{p}\right)$ and $\left(\left[f_{2}\right], \underline{p}\right)$ are visibly equivalent.

We set $\operatorname{gr}_{\infty}(f: S \rightarrow \Sigma):=\left[\operatorname{gr}_{\infty}(f): R \rightarrow \operatorname{gr}_{\infty}(\Sigma)\right]$, on the bounded part $\overline{\mathcal{T}}(S) \subset$ $\tilde{\mathcal{T}}(S)$. On the other hand, if $\tilde{w} \in|\mathfrak{A}(S)|_{\infty}$ represents a point at infinity of $\widetilde{\mathcal{T}}(S)$, then we define $\left(\operatorname{gr}_{\infty}, \mathcal{L}\right)(\tilde{w}):=\left(\boldsymbol{W}_{\mathrm{HMT}}^{-1}(\tilde{w}), \infty\right)$.

The following is our key result.

Theorem 5.4. The map $\left(\mathrm{gr}_{\infty}, \mathcal{L}\right)$ is a $\Gamma(S)$-equivariant homeomorphism that preserves the topological types and whose restriction to each topological stratum of the finite part and to each simplex of $|\mathfrak{A}(S)|_{\infty}$ is a real-analytic diffeomorphism.

Corollary 5.5. (a) The induced map $\widetilde{\mathcal{M}}(S) \rightarrow \overline{\mathcal{M}}(R, x) \times \Delta^{n-1} \times[0, \infty] / \sim$ is a homeomorphism, which is real-analytic on $\widehat{\mathcal{M}}(S)$ and piecewise real-analytic on $|\mathfrak{A}(S)|_{\infty} / \Gamma(S)$.

(b) Let $\widehat{\mathcal{T}}^{\text {vis }}(R, x)$ (resp. $\widehat{\mathcal{M}}^{\text {vis }}(R, x)$ ) be obtained from $\overline{\mathcal{T}}(R, x) \times \Delta^{n-1}$ (resp. $\left.\overline{\mathcal{M}}(R, x) \times \Delta^{n-1}\right)$ by identifying visibly equivalent surfaces. Then the induced $\overline{\mathcal{T}}^{a}(S) \rightarrow \widehat{\mathcal{T}}^{\text {vis }}(R, x) \times[0, \infty]$ and $\overline{\mathcal{M}}^{a}(S) \rightarrow \widehat{\mathcal{M}}^{\text {vis }}(R, x) \times[0, \infty]$ are homeomorphisms.

We can summarize our results in the following commutative diagram:

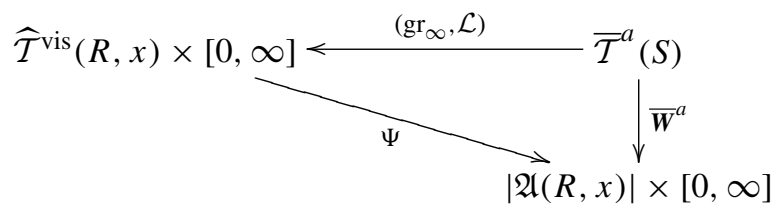


in which $\Psi=\overline{\boldsymbol{W}}^{a} \circ\left(\mathrm{gr}_{\infty}, \mathcal{L}\right)^{-1}$ and all maps are $\Gamma(R, x)$-equivariant homeomorphisms. For every $t \in[0, \infty]$, denote by $\Psi_{t}: \widehat{\mathcal{T}}^{\mathrm{vis}}(R, x) \rightarrow|\mathfrak{A}(R, x)|$ the restriction of $\Psi$ to $\widehat{\mathcal{T}}^{\mathrm{vis}}(R, x) \times\{t\}$ followed by the projection onto $|\mathfrak{A}(R, x)|$.

Corollary 5.6. $\Psi_{t}$ is a continuous family of $\Gamma(R, x)$-equivariant triangulations of $\widehat{\mathcal{T}}^{\mathrm{vis}}(R, x)$, whose extremal cases are Bowditch-Epstein/Penner's for $t=0$ and HarerMumford-Thurston's for $t=\infty$.

The continuity of $\left(\mathrm{gr}_{\infty}, \mathcal{L}\right)$ is proven in Appendix D Lemma C.1 a) ensures that this map is proper. In order to prove Theorem 5.4, we need to show that the restriction of $\left(\mathrm{gr}_{\infty}, \mathcal{L}\right)$ to each stratum is bijective onto its image, and so that $\left(\mathrm{gr}_{\infty}, \mathcal{L}\right)$ is bijective.

\subsection{Bijectivity of $\left(\mathrm{gr}_{\infty}, \mathcal{L}\right)$}

The bijectivity at infinity (namely, for $\|\mathcal{L}\|=\infty$ ) follows from Theorem 5.2. Thus, let us select a (possibly empty) system of curves $\gamma=\left\{\gamma_{1}, \ldots, \gamma_{k}\right\}$ on $S$ and consider the stratum $\mathcal{S}(\boldsymbol{\gamma}) \subset \widehat{\mathcal{T}}(S)$ in which $\|\mathcal{L}\|<\infty$ and $\ell_{\gamma_{i}}=0$ for every $i$.

To show that $\left(\mathrm{gr}_{\infty}, \mathcal{L}\right)$ gives a bijection of $\mathcal{S}(\gamma)$ onto its image, it is sufficient to work separately on each component of $S \backslash \boldsymbol{\gamma}$. Thus, we can reduce to the case in which $\gamma_{i}=C_{i} \subset \partial S$ and $\operatorname{gr}_{\infty}$ glues a cylinder at the boundary components $C_{k+1}, \ldots, C_{n}$. Hence, we are reduced to showing that the grafting map

$$
\operatorname{gr}_{\infty}^{\prime}: \mathcal{T}(S)(\underline{p}) \rightarrow \mathcal{T}(S)(0)
$$

is bijective for every $p_{k+1}, \ldots, p_{n} \in \mathbb{R}_{+}$, where $p_{1}=\cdots=p_{k}=0$. We already know that $\operatorname{gr}_{\infty}^{\prime}$ is continuous and proper; we will show that it is a local homeomorphism by adapting the argument of [SW02]. Here we describe what considerations are needed to make their proof work in our case.

Remark 5.7. Here we are using the notation $\mathcal{T}(S)(0)$ instead of $\mathcal{T}(R, x)$ because we want to stress that we are regarding $\operatorname{gr}_{\infty}(\Sigma)$ as a hyperbolic surface, with the metric coming from the uniformization.

The grafted metrics are $C^{1,1}$ but the map $\mathrm{gr}_{\infty}^{\prime}$ is real-analytic. In fact, given a realanalytic $\operatorname{arc}\left[f_{t}: S \rightarrow \Sigma_{t}\right]$ in $\mathcal{T}(S)(\underline{p})$ and choosing representatives $f_{t}$ so that $f_{0} \circ f_{t}^{-1}$ : $\Sigma_{t} \rightarrow \Sigma_{0}$ is an isometry on the boundary components $C_{k+1, t}, \ldots, C_{n, t}$ and harmonic in the interior with respect to the hyperbolic metrics (so that the hyperbolic metrics pull back to a real-analytic family $\sigma_{t}$ on $S$ ), we can choose the grafted maps $\operatorname{gr}_{\infty}^{\prime}\left(f_{t}\right): S \rightarrow \Sigma_{t}$ so that $\operatorname{gr}_{\infty}^{\prime}\left(f_{0}\right) \circ \operatorname{gr}_{\infty}^{\prime}\left(f_{t}\right)^{-1}$ extend $f_{0} \circ f_{t}^{-1}$ as isometries on the cylinders $\tilde{C}_{i, t}:=$ $C_{i, t} \times[0, \infty)$. Hence, the family of metrics $\operatorname{gr}_{\infty}^{\prime}\left(\sigma_{t}\right)$ on $S$, obtained by pulling the Thurston metric back via $\operatorname{gr}_{\infty}^{\prime}\left(f_{t}\right)$, is real-analytic in $t$ and so the $\operatorname{arc}\left[\operatorname{gr}_{\infty}^{\prime}\left(f_{t}\right)\right]$ in $\mathcal{T}(S)(0)$ is realanalytic.

Thus, it is sufficient to show that the differential $d \mathrm{gr}_{\infty}^{\prime}$ is injective at every point of $\mathcal{T}(S)(p)$.

Given a real-analytic one-parameter family $f_{t}: S \rightarrow \Sigma_{t}$ corresponding to a tangent vector $v \in T_{\left[f_{0}\right]} \mathcal{T}(S)(\underline{p})$, assume that the grafted family $\left[\operatorname{gr}_{\infty}^{\prime}\left(f_{t}\right): S \rightarrow \operatorname{gr}_{\infty}^{\prime}\left(\Sigma_{t}\right)\right]$ defined above determines the zero tangent vector in $T_{\left[\mathrm{gr}_{\infty}^{\prime}\left(f_{0}\right)\right]} \mathcal{T}(S)(0)$. 
Let $\operatorname{gr}_{\infty}^{\prime}\left(\sigma_{t}\right)$ be the pull-back via $f_{t}$ of the hyperbolic metric of $\Sigma_{t}$ and construct the harmonic representative $F_{t}:\left(S, \operatorname{gr}_{\infty}^{\prime}\left(\sigma_{t}\right)\right) \rightarrow\left(S, \operatorname{gr}_{\infty}^{\prime}\left(\sigma_{0}\right)\right)$ in the class of the identity as follows.

Give orthonormal coordinates $(x, y)$ to the cylinder $\tilde{C}_{i, t} \cong C_{i, t} \times[0, \infty)$ that is glued at the boundary component $C_{i, t} \subset \Sigma_{t}$ for $i=k+1, \ldots, n$, in such a way that $x$ is the arc-length parameter of the circumferences and $y \in[0, \infty)$.

Remark 5.8. The $(x, y)$ coordinates can be extended to an orthogonal system in a small hyperbolic collar of $C_{i, t}$ in such a way that $y$ is the arc-length parameter along the geodesics $\{x=$ const $\}$. Thus, for $y \in(-\varepsilon, 0)$, the metric looks like $\cosh (y)^{2} d x^{2}+d y^{2}=$ $d x^{2}+d y^{2}+O\left(\varepsilon^{2}\right)$.

Define the $M$-ends of $\operatorname{gr}_{\infty}^{\prime}\left(\Sigma_{t}\right)$ to be the subcylinders $C_{i, t} \times[M, \infty)$ for $i=k+1$, $\ldots, n$ and use the same terminology for their images in $S$ via $\operatorname{gr}_{\infty}^{\prime}\left(f_{t}\right)^{-1}$.

For every $t$, let $\mathfrak{F}_{t}:=\bigcup_{M>0} \mathfrak{F}_{t}(M)$ where $\mathfrak{F}_{t}(M)$ is the set of $C^{1,1}$ diffeomorphisms $g_{t}:\left(S, \operatorname{gr}_{\infty}\left(\sigma_{t}\right)\right) \rightarrow\left(S, \operatorname{gr}_{\infty}\left(\sigma_{0}\right)\right)$ homotopic to the identity, such that $g_{t}$ isometrically preserves the $M$-ends. Clearly, $\mathfrak{F}_{t}(M) \subseteq \mathfrak{F}_{t}\left(M^{\prime}\right)$ if $M \leq M^{\prime}$.

Let $e\left(g_{t}\right)=\frac{1}{2}\left\|\nabla g_{t}\right\|^{2}$ be the energy density of $g_{t}$,

$$
\mathcal{H}\left(g_{t}\right)=\left\|d g_{t}\left(\partial_{z}\right)\right\|^{2} \frac{d z d \bar{z}}{\operatorname{gr}_{\infty}^{\prime}\left(\sigma_{t}\right)}
$$

where $z$ is a local conformal coordinate on $\left(S, \operatorname{gr}_{\infty}^{\prime}\left(\sigma_{t}\right)\right)$, and $\mathcal{J}\left(g_{t}\right)$ the Jacobian determinant of $g_{t}$, so that $e\left(g_{t}\right)=2 \mathcal{H}\left(g_{t}\right)-\mathcal{J}\left(g_{t}\right)$. Notice that, if $g_{t}$ is an oriented diffeomorphism, then $0<\mathcal{J}\left(g_{t}\right) \leq \mathcal{H}\left(g_{t}\right) \leq e\left(g_{t}\right)$ at each point.

Define also the reduced quantities $\tilde{e}\left(g_{t}\right)=e\left(g_{t}\right)-1, \tilde{\mathcal{H}}\left(g_{t}\right)=\mathcal{H}\left(g_{t}\right)-1$ and $\tilde{\mathcal{J}}\left(g_{t}\right)=$ $\mathcal{J}\left(g_{t}\right)-1$, so that the reduced energy

$$
\tilde{E}\left(g_{t}\right):=\int_{S} \tilde{e}\left(g_{t}\right) \operatorname{gr}_{\infty}^{\prime}\left(\sigma_{t}\right)
$$

is well-defined for every $g_{t} \in \mathfrak{F}_{t}$. For instance, the identity map on $S$ belongs to $\mathfrak{F}_{t}(0)$ and its reduced energy is $E\left(f_{0} \circ f_{t}^{-1}\right)-2 \pi \chi(S)$.

As $\operatorname{gr}_{\infty}^{\prime}\left(\sigma_{0}\right)$ is nonpositively curved, the map $F_{t, M}$ of least energy in $\mathfrak{F}_{t}(M)$ is harmonic away from the $M$-ends and so is an oriented diffeomorphism. Thus,

$$
0=\int_{S} \tilde{\mathcal{J}}\left(F_{t, M}\right) \operatorname{gr}_{\infty}^{\prime}\left(\sigma_{t}\right) \leq \int_{S} \tilde{\mathcal{H}}\left(F_{t, M}\right) \operatorname{gr}_{\infty}^{\prime}\left(\sigma_{t}\right) \leq \tilde{E}\left(F_{t, M}\right)
$$

Hence, $\left\{F_{t, M}\right\}_{M}$ converges uniformly on compact subsets to the map $F_{t}$ of least (reduced) energy in $\mathfrak{F}_{t}$, which is unique. Set $\tilde{\mathcal{H}}_{t}:=\tilde{\mathcal{H}}\left(F_{t}\right)$ and similarly $\tilde{e}_{t}=\tilde{e}\left(F_{t}\right)$.

Following Scannell-Wolf (but noticing that the roles of $x$ and $y$ here are exchanged compared to their paper), one can show that

- the family $\left\{F_{t}\right\}$ is real-analytic in $t$,

- for every small $t$, the map $F_{t}$ is (locally) $C^{2, \alpha}$ on $S$; so is the vector field $\dot{F}:=\dot{F}_{0}$ (hence, the analyticity of $F_{t}$ implies that $\tilde{\mathcal{H}}_{t}$ and $\tilde{e}_{t}$ are real-analytic in $t$ too), 
- the function $\dot{\tilde{\mathcal{H}}}:=\dot{\tilde{\mathcal{H}}}_{0}$ is locally Lipschitz away from the cusps and it is harmonic on the flat cylinders,

- along every $C_{k+1}, \ldots, C_{n}$, we have

$$
V=-\frac{1}{2}\left(\left(\partial_{y} \dot{\tilde{\mathcal{H}}}\right)_{+}-\left(\partial_{y} \dot{\tilde{\mathcal{H}}}\right)_{-}\right)
$$

where $V(x, y)$ is a harmonic function defined on the cylinders $\tilde{C}_{i, 0}$ (and on first-order thickenings of $C_{i, 0}$ ) that can be identified with the $y$-component of $\dot{F}$, and $w(x, 0)_{+}$ simply means $\lim _{y \rightarrow 0^{+}} w(x, y)$.

- $V_{y}=\frac{1}{2} \dot{\mathcal{H}}+c_{i}$ on each $\tilde{C}_{i}$, where $c_{i}$ is a constant that may depend on the cylinder.

From now on, let all line integrals be with respect to the arc-length parameter $d x$ and all surface integrals with respect to $\operatorname{gr}_{\infty}^{\prime}\left(\sigma_{0}\right)$. Notice that

$$
\int_{S} \dot{\mathcal{H}}=\int_{S} \dot{\tilde{\mathcal{H}}}=\lim _{t \rightarrow 0} \frac{1}{t} \int_{S} \tilde{\mathcal{H}}_{t}
$$

because $\tilde{\mathcal{H}}_{0}=0$. As the integral on the right is a real-analytic function of $t$ which vanishes at $t=0$, we conclude that $\dot{\tilde{\mathcal{H}}}$ is integrable and so is $\dot{\tilde{e}}$.

On the other hand, $\dot{\tilde{e}}=\left.\frac{1}{2} \frac{\partial}{\partial t}\left\|\nabla F_{t}\right\|^{2}\right|_{t=0}=\partial_{x}\left(\dot{F} \cdot \partial_{x}\right)+V_{y}$. As $\partial_{x}\left(\dot{F} \cdot \partial_{x}\right)$ has zero average, $V_{y}$ is integrable too and all constants $c_{i}$ are 0 . Thus, $V$ and $\dot{\tilde{\mathcal{H}}}$ decay at least as $\exp \left(-2 \pi y / p_{i}\right)$ on $\tilde{C}_{i, 0}$ and we can write

$$
0=\int_{\tilde{C}_{i, 0}} V \Delta V=-\int_{\tilde{C}_{i, 0}}\|\nabla V\|^{2}+\int_{C_{i, 0}} V \partial_{n} V .
$$

Moreover,

$$
0 \leq \int_{\tilde{C}_{i, 0}}\|\nabla V\|^{2}=\int_{C_{i, 0}} V_{y} V=\frac{1}{2} \int_{C_{i, 0}} \dot{\tilde{\mathcal{H}}} V
$$

On the other hand, multiplying by $\dot{\tilde{\mathcal{H}}}=\dot{\mathcal{H}}$ and integrating by parts the linearized equation

$$
\left(\Delta_{\mathrm{gr}_{\infty}^{\prime}\left(\sigma_{0}\right)}+2 K_{0}\right) \dot{\mathcal{H}}=0
$$

where $K_{0}$ is the curvature of $\operatorname{gr}_{\infty}^{\prime}\left(\sigma_{0}\right)$, we obtain

$$
\left\{\begin{array}{l}
0 \leq \int_{\tilde{C}_{i, 0}}\|\nabla \dot{\mathcal{H}}\|^{2}=\int_{C_{i, 0}} \dot{\mathcal{H}}\left(\partial_{n} \dot{\mathcal{H}}\right)_{+}, \\
0 \leq \int_{S_{\text {hyp }}}\left(\|\nabla \dot{\mathcal{H}}\|^{2}+2|\dot{\mathcal{H}}|^{2}\right)=-\sum_{i=k+1}^{n} \int_{C_{i, 0}} \dot{\mathcal{H}}\left(\partial_{n} \dot{\mathcal{H}}\right)_{-}
\end{array}\right.
$$

where $S_{\text {hyp }}$ is the $\operatorname{gr}_{\infty}^{\prime}\left(\sigma_{0}\right)$-hyperbolic part of $S$. 
From $0 \leq \sum_{i=k+1}^{n} \int_{C_{i, 0}} \dot{\mathcal{H}}\left(\left(\partial_{y} \dot{\mathcal{H}}\right)_{+}-\left(\partial_{y} \dot{\mathcal{H}}\right)_{-}\right)$, we finally get

$$
0 \leq 2 \int_{S}\left(\|\nabla \dot{\mathcal{H}}\|^{2}-2 K\|\dot{\mathcal{H}}\|^{2}\right)=-\sum_{i=k+1}^{n} \int_{C_{i, 0}} \dot{\mathcal{H}} V
$$

Combining (1) and 22), we obtain

$$
\int_{C_{i, 0}} \dot{\mathcal{H}} V=0 \quad \forall i=k+1, \ldots, n,
$$

and so $\dot{\mathcal{H}}=0$ on $S$.

Hence, away from the cusps, $F_{t}$ is a $(1+o(t))$-isometry between $\operatorname{gr}_{\infty}^{\prime}\left(\sigma_{t}\right)$ and $\operatorname{gr}_{\infty}^{\prime}\left(\sigma_{0}\right)$ and one can easily conclude that $\sigma_{t}$ and $\sigma_{0}$ are $(1+o(t))$-isometric too.

\subsection{More on infinitely grafted structures}

5.6.1. Projective structures. Consider a Riemann surface $R$ without boundary and of genus at least 2. A projective structure on a marked surface $\left[f: R \rightarrow R^{\prime}\right]$ is an equivalence class of holomorphic atlases $\mathfrak{U}=\left\{f_{i}: U_{i} \rightarrow \mathbb{C P}^{1} \mid R^{\prime} \supset U_{i}\right.$ open $\}$ for $R^{\prime}$ such that the transition functions belong to $\operatorname{Aut}\left(\mathbb{C P}^{1}\right) \cong \operatorname{PSL}(2, \mathbb{C})$, that is, $\left.f_{i}\right|_{U_{i} \cap U_{j}}$ and $\left.f_{j}\right|_{U_{i} \cap U_{j}}$ are projectively equivalent.

Given two projective structures, represented by maximal atlases $\mathfrak{U}$ and $\mathfrak{V}$, on the same $\left[f: R \rightarrow R^{\prime}\right] \in \mathcal{T}(R)$ and a point $p \in R^{\prime}$, we want to measure how charts of $\mathfrak{U}$ are not projectively equivalent to charts in $\mathfrak{V}$ around $p$. So, let $f: U \rightarrow \mathbb{C P}^{1}$ be a chart in $\mathfrak{U}$ and $g: U \rightarrow \mathbb{C P}^{1}$ a chart in $\mathfrak{V}$, with $U \subset R^{\prime}$. There exists a unique $\sigma \in \operatorname{PSL}(2, \mathbb{C})$ such that $f$ and $\sigma \circ g$ agree up to second order at $p$. Then $(f-\sigma \circ g)^{\prime \prime \prime}: T_{p} U \rightarrow T_{f(p)} \mathbb{C P}^{1}$ is a homogeneous cubic map and $f^{\prime}(p)^{-1} \circ(f-\sigma \circ g)^{\prime \prime \prime}$ is a homogeneous cubic endomorphism of $T_{p} U$, and so an element $S(f, g)(p)$ of $\left(T_{p}^{*} U\right)^{\otimes 2}$. The holomorphic quadratic differential $S(\mathfrak{U}, \mathfrak{V})$ on $R^{\prime}$ is called the Schwarzian derivative. It is known that, given a $\mathfrak{U}$ and a holomorphic quadratic differential $\varphi \in \mathcal{Q}_{R^{\prime}}$, there exists a unique projective structure $\mathfrak{V}$ on $R^{\prime}$ such that $S(\mathfrak{U}, \mathfrak{V})=\varphi$.

Thus, the natural projection $\pi: \mathcal{P}(R) \rightarrow \mathcal{T}(R)$ from the set $\mathcal{P}(R)$ of projective structures on $R$ (up to isotopy) to the Teichmüller space of $R$ is a principal $\mathcal{Q}$-bundle, where $\mathcal{Q} \rightarrow \mathcal{T}(R)$ is the bundle of holomorphic quadratic differentials.

Assume now that $R$ is compact. The grafting map gr : $\mathcal{T}(R) \times \mathcal{M L}(R) \rightarrow \mathcal{T}(R)$ admits a lifting

$$
\mathrm{Gr}: \mathcal{T}(R) \times \mathcal{M L}(R) \stackrel{\sim}{\rightarrow} \mathcal{P}(R)
$$

which is a homeomorphism (Thurston [KT92]) and such that $\mathrm{Gr}_{0}$ corresponds to the Poincaré structure. We recall that a surface with projective structure comes endowed with a Thurston $C^{1,1}$ metric: in particular, if $\lambda=c_{1} \gamma_{1}+\cdots+c_{n} \gamma_{n}$ is a multi-curve on $R$, and $R^{\prime}$ is an $R$-marked hyperbolic surface, then $\operatorname{Gr}_{\lambda}\left(R^{\prime}\right)$ is made of a hyperbolic piece, isometric to $R^{\prime} \backslash \operatorname{supp}(\lambda)$, and $n$ flat cylinders homotopic to $\gamma_{i}$ and of height $c_{i}$.

It is a general fact that $\mathrm{Gr}_{\lambda}$ determines a real-analytic section of $\pi$ for all $\lambda \in \mathcal{M L}$. 
5.6.2. A compactification of $\mathcal{P}(R)$. The homeomorphism $\mathcal{T}(R) \times \mathcal{M L}(R) \cong \mathcal{P}(R)$ shows that sequences $\left\{\left(\left[f_{m}: R \rightarrow R_{m}^{\prime}\right], \lambda_{m}\right)\right\}$ in $\mathcal{P}(R)$ can diverge in two "directions".

Dumas [Dum06] provides a grafting compactification of $\mathcal{P}(R)$ by separately compactifying $\mathcal{T}(R)$ and $\mathcal{M L}(R)$. In particular, he defines $\overline{\mathcal{P}}(R):=\overline{\mathcal{T}}^{\mathrm{Th}}(R) \times \overline{\mathcal{M L}}(R)$, where $\overline{\mathcal{T}}^{\text {Th }}(R)=\mathcal{T}(R) \cup \mathbb{P} \mathcal{M L}(R)$ is Thurston's compactification (see [FLP79]) and $\overline{\mathcal{M L}}(R)=\mathcal{M L}(R) \cup \mathbb{P} \mathcal{M L}(R)$ is the natural projective compactification of $\mathcal{M L}(R)$.

In order to describe the asymptotic properties of $\overline{\mathcal{P}}(R)$, we recall the following wellknown result.

Theorem 5.9 ([[HM79]). The map

$$
\Lambda: \mathcal{Q} \rightarrow \mathcal{T}(R) \times \mathcal{M L}(R)
$$

defined as $\left(\left[f: R \rightarrow R^{\prime}\right], \varphi\right) \mapsto\left(\left[f: R \rightarrow R^{\prime}\right], f^{*} \Lambda_{R^{\prime}}(\varphi)\right)$ is a homeomorphism, where $\Lambda_{R^{\prime}}(\varphi)$ is the measured lamination on $R^{\prime}$ obtained by straightening the (measured) horizontal foliation of $\varphi$.

The antipodal map is the homeomorphism

$$
\iota: \mathcal{T}(R) \times \mathcal{M L}(R) \rightarrow \mathcal{T}(R) \times \mathcal{M L}(R)
$$

given by $\iota\left(\left[f: R \rightarrow R^{\prime}\right], \lambda\right)=\Lambda\left(-\Lambda^{-1}([f], \lambda)\right)$. The following result shows that the restriction $\iota_{f}: \mathcal{M L}(R) \rightarrow \mathcal{M L}(R)$ of $i$ to a certain point $[f] \in \mathcal{T}(R)$ controls the asymptotic behaviour of $\pi^{-1}(f)$.

Theorem 5.10 ([Dum06], [Dum07b]). Let $\left\{\left(\left[f_{m}: R \rightarrow R_{m}\right], \lambda_{m}\right)\right\} \subset \mathcal{T}(R) \times \mathcal{M L}(R)$ be a diverging sequence such that $\pi \circ \operatorname{Gr}_{\lambda_{m}}\left(f_{m}\right)=\left[f: R \rightarrow R^{\prime}\right]$. The following are equivalent:

(1) $\lambda_{m} \rightarrow[\lambda]$ in $\overline{\mathcal{M L}}(R)$, where $[\lambda] \in \mathbb{P} \mathcal{M L}(R)$,

(2) $\left[f_{m}\right] \rightarrow\left[\iota_{f}(\lambda)\right]$ in $\overline{\mathcal{T}}^{\mathrm{Th}}(R)$,

(3) $\Lambda_{f}\left(-S\left(\mathrm{Gr}_{\lambda_{m}}\left(f_{m}\right)\right)\right) \rightarrow[\lambda]$ in $\overline{\mathcal{M L}}(R)$,

(4) $\Lambda_{f}\left(\boldsymbol{S}\left(\mathrm{Gr}_{\lambda_{m}}\left(f_{m}\right)\right)\right) \rightarrow\left[i_{f}(\lambda)\right]$ in $\overline{\mathcal{M L}}(R)$, where the Schwarzian derivative is considered with respect to the Poincaré structure.

When this happens, we also have $\left[\boldsymbol{H}\left(\kappa_{m}\right)\right] \rightarrow\left[\Lambda^{-1}\left(R^{\prime}, \lambda\right)\right]$ in $\mathbb{P} L^{1}\left(R^{\prime}, K^{\otimes 2}\right)$, where $\boldsymbol{H}\left(\kappa_{m}\right)$ is the Hopf differential of the collapsing map $\kappa_{m}: R^{\prime} \rightarrow R_{m}$.

We recall that, if $\lambda_{m}$ is a multi-curve $c_{1} \gamma_{1}+\cdots+c_{n} \gamma_{n}$, then $\kappa_{m}$ collapses the $n$ grafted cylinders onto the respective geodesics and is the identity elsewhere. Thus, if the $j$-th flat cylinder is isometric to $\left[0, \ell_{j}\right] \times\left[0, c_{j}\right] /(0, y) \sim\left(\ell_{j}, y\right)$, then $\boldsymbol{H}\left(\kappa_{m}\right)$ restricts to $d z^{2}$ on the grafted cylinders and vanishes on the remaining hyperbolic portion of $R^{\prime}$.

Remark 5.11. The theorem implies that the boundary of $\pi^{-1}(f) \subset \overline{\mathcal{P}}(R)$ is exactly the graph of the projectivization of $i_{f}$. 
5.6.3. Surfaces with infinitely grafted ends. We can adapt Theorem 5.10 to our situation, when we restrict our attention to smooth hyperbolic surfaces with large boundary.

Let $S$ be a compact oriented surface of genus $g$ with boundary components $C_{1}, \ldots$, $C_{n}$ (and $\left.\chi(S)=2-2 g-n<0\right)$ and let $d S$ be its double.

Theorem 5.12. Let $\left\{\left[f_{m}: S \rightarrow \Sigma_{m}\right]\right\} \subset \mathcal{T}(S)$ be a sequence such that $\left(\mathrm{gr}_{\infty}, \mathcal{L}\right)\left(f_{m}\right)=$ $\left(\left[f:(R, x) \rightarrow\left(R^{\prime}, x^{\prime}\right)\right], \underline{p}_{m}\right) \in \mathcal{T}(R, x) \times \mathbb{R}_{+}^{n}$. The following are equivalent:

(1) $\underline{p}_{m} \rightarrow(\underline{p}, \infty)$ in $\Delta^{n-1} \times(0, \infty]$,

(2) $\left[f_{m}\right] \rightarrow \tilde{w}$ in $\overline{\mathcal{T}}^{a}(S)$, where $\tilde{w}$ is the projective multi-arc associated to the vertical foliation of the Jenkins-Strebel differential $\varphi_{\mathrm{JS}}$ on $\left(R^{\prime}, x^{\prime}\right)$ with weights $\underline{p}$ (see Theorem 5.2.

When this happens, we also have

(a) $4\left\|p_{m}\right\|^{-2} \boldsymbol{H}\left(\kappa_{m}\right) \rightarrow \varphi_{\mathrm{JS}}$ in $L_{\mathrm{loc}}^{1}\left(\dot{R}^{\prime}\right)$, where $\boldsymbol{H}\left(\kappa_{m}\right)$ is the Hopf differential of the collapsing map $\kappa_{m}: R^{\prime} \rightarrow \Sigma_{m}$,

(b) with respect to the Poincaré projective structure on $\Sigma_{m}^{\circ}, 2\left\|p_{m}\right\|^{-2} S\left(\operatorname{Gr}_{\infty}\left(f_{m}\right)\right) \rightarrow$ $-\varphi_{\mathrm{JS}}$ in $H^{0}\left(R^{\prime}, K\left(x^{\prime}\right)^{\otimes 2}\right)$.

Remark 5.13. We have denoted by $\operatorname{Gr}_{\infty}\left(f_{m}: S \rightarrow \Sigma_{m}\right)$ the ( $S$-marked) surface with projective structure obtained from $\Sigma_{m}$ by grafting cylinders of infinite length at its ends. This is a somewhat "very exotic" projective structure, whose developing map wraps infinitely many times around $\mathbb{C P}^{1}$. Its Schwarzian with respect to the Poincaré structure has double poles at the cusps.

We have already shown that (1) and (2) are equivalent to each other.

Lemma 5.14. For every compact $K \subset \dot{R}^{\prime}$, there exists $t_{0}>0$ such that $K \subset K_{m}^{t}:=$ $\mathrm{gr}_{t\left\|p_{m}\right\| \partial \Sigma_{m}}\left(\Sigma_{m}\right) \subset \dot{R}^{\prime}$ for every $t \geq t_{0}$.

Proof. Let $z \in K$. There exists $c_{z}>0$ such that the extremal length of a curve inside $\dot{R}^{\prime}$ isotopic to $C_{j}$ that separates a disk containing $\left\{z, x_{j}\right\}$ from the remaining surface is at least $c_{z}$ for every $j$. On the other hand, the extremal length of $C_{j}$ inside $K_{m}^{t}$ is at most $1 / t$. Hence, there is a neighbourhood $U_{z}$ of $z$ such that $U_{z} \subset K_{m}^{t}$ for every $t>1 / c_{z}$. As $K$ is compact, there exist $z_{1}, \ldots, z_{i}$ such that $U_{z_{1}}, \ldots, U_{z_{i}}$ cover $K$ and so $t_{0}>$ $\max \left\{1 / c_{z_{1}}, \ldots, 1 / c_{z_{i}}\right\}$ works.

The proof of (a) of Theorem 5.12 follows [Dum06] (see also [Dum07a]) with minor modifications:

- Let $h_{m}: \dot{R}^{\prime} \rightarrow \Sigma_{m}$ be the harmonic map homotopic to $\kappa_{m}$, that is the limit as $s \rightarrow \infty$ of the harmonic maps $h_{m}^{s}: \operatorname{gr}_{s \partial \Sigma_{m}}\left(\Sigma_{m}\right) \rightarrow \Sigma_{m}$ that restrict to isometries at the boundary; we clearly have

$$
\left\|\boldsymbol{H}\left(h_{m}\right)-\boldsymbol{H}\left(\kappa_{m}\right)\right\|_{L^{1}(K)} \leq\left\|\boldsymbol{H}\left(h_{m}\right)-\boldsymbol{H}\left(\kappa_{m}\right)\right\|_{L^{1}\left(K_{m}^{t}\right)}
$$

and $E_{K_{m}^{t}}\left(h_{m}\right)<E_{K_{m}^{t}}\left(\kappa_{m}\right)=2 \pi|\chi(S)|+t\left\|p_{m}\right\|^{2} / 2 \leq E_{K_{m}^{t}}\left(h_{m}\right)+2 \pi|\chi(S)|$, where $E_{K_{m}^{t}}$ is the integral of the energy density on $K_{m}^{t}$. 
- The statement that $\left[\boldsymbol{H}\left(h_{m}\right)\right] \rightarrow[\varphi]$ as $m \rightarrow \infty$ is basically proven by Wolf in [Wol89]; in fact, the considerations involved in his argument do not require the integrability of $\boldsymbol{H}\left(h_{m}\right)$ or $\varphi$ over the whole $\dot{R}^{\prime}$ : rescaling the Hopf differential in order to have the right boundary lengths, one obtains

$$
4\left\|p_{m}\right\|^{-2} \boldsymbol{H}\left(h_{m}\right) \rightarrow \varphi \quad \text { in } L_{\mathrm{loc}}^{1}\left(\dot{R}^{\prime}\right) .
$$

- The local estimate

$$
\left\|\boldsymbol{H}\left(h_{m}\right)-\boldsymbol{H}\left(\kappa_{m}\right)\right\|_{L^{1}(K)} \leq \sqrt{2\left(E_{K}\left(h_{m}\right)-E_{K}\left(\kappa_{m}\right)\right)}\left(\sqrt{E_{K}\left(h_{m}\right)}+\sqrt{E_{K}\left(\kappa_{m}\right)}\right)
$$

is obtained in the proof of Proposition 2.6.3 of [KS93].

- One easily concludes, because $\left\|\boldsymbol{H}\left(h_{m}\right)-\boldsymbol{H}\left(\kappa_{m}\right)\right\|_{L^{1}\left(K_{m}^{t}\right)}=O\left(\left\|p_{m}\right\| \sqrt{t}\right)$ and $\left\|\boldsymbol{H}\left(h_{m}\right)\right\|_{L^{1}\left(K_{m}^{t}\right)}=O\left(\left\|p_{m}\right\|^{2} t\right)$.

Assertion (b) is also basically proven in [Dum07b] up to minor considerations:

- Let $\rho$ be the hyperbolic metric on $\dot{R}^{\prime}$ and $\rho_{m}$ the Thurston metric on $\operatorname{Gr}_{\infty}\left(\Sigma_{m}\right) \cong \dot{R}^{\prime}$; moreover, let $\beta_{m}$ be the Schwarzian tensor $\beta\left(\rho, \rho_{m}\right)=\left[\operatorname{Hess}_{\rho}\left(\sigma_{m}\right)-d \sigma_{m} \otimes d \sigma_{m}\right]^{2,0}$, where $\sigma_{m}=\sigma\left(\rho, \rho_{m}\right)=\log \left(\rho_{m} / \rho\right)$.

- The decomposition ([Dum07b, Theorem 7.1])

$$
\boldsymbol{S}\left(\operatorname{Gr}_{\infty}\left(\Sigma_{m}\right)\right)=2 \beta_{m}-2 \boldsymbol{H}\left(\kappa_{m}\right)
$$

(where $S$ is computed with respect to the Poincare structure on $\dot{R}^{\prime}$ ) still holds, because it relies on local considerations.

- Let $K$ be the compact subsurface of $\dot{R}^{\prime}$ obtained by removing all $n$ horoballs of circumference $1 / 4$ at $x^{\prime}$. Moreover, let $\rho_{\underline{p}}$ be the Thurston metric on $\Sigma$ obtained by grafting infinite flat cylinders at the boundary of $\left(\mathrm{gr}_{\infty}, \mathcal{L}\right)^{-1}([f], \underline{p})$ and write $\hat{\rho}_{\underline{p}}:=$ $\left(1+\|\underline{p}\|^{2}\right) \rho_{\underline{p}}$ for the normalized metrics. The set $\mathcal{N}=\left\{\hat{\rho}_{\underline{p}} \mid \underline{p} \in \Delta^{n-1} \times[0, \infty]\right\}$ is compact in $L^{\infty}(K)$. Thus, $\left\|\hat{\rho}_{\underline{p}} / \rho\right\|_{L^{\infty}(K)}<c$ and all restrictions to $K$ of metrics in $\mathcal{N}$ are pairwise Hölder equivalent with factor and exponent dependent on $\dot{R}^{\prime}$ only (same proof as in Theorem 9.2 of [Dum07b]).

- The same estimates of [Dum07b] give (Theorem 11.4)

$$
\left\|\beta_{m}\right\|_{L^{1}\left(D_{\delta / 4}, \rho\right)} \leq c
$$

where $c$ depends on $\dot{R}^{\prime}$ and $\delta$.

- All norms are equivalent on $H^{0}\left(R^{\prime}, K\left(x^{\prime}\right)^{\otimes 2}\right)$, so we consider the $L^{1}$ norm on $K \subset \dot{R}^{\prime}$ and we observe that $\|\psi\|_{L^{1}\left(D_{\delta / 4}, \rho\right)} \leq c^{\prime}\|\psi\|_{L^{1}(K)}$ for any $\rho$-ball of radius $\delta / 4$ embedded in $K$.

- By Lemma 5.14, there exists $t_{0}$ (depending only on $\dot{R}^{\prime}$ and $K$ ) such that $K \subset K_{m}^{t_{0}}$ for all $m$. Thus,

$$
\begin{aligned}
& \| \boldsymbol{S}\left(\mathrm{Gr}_{\infty}\left(\Sigma_{m}\right)\right)+\varphi_{\mathrm{JS}} \|_{L^{1}(K)} \\
& \quad \leq c_{1}\left\|2 \boldsymbol{S}\left(\mathrm{Gr}_{\infty}\left(\Sigma_{m}\right)\right)+4 \boldsymbol{H}\left(\kappa_{m}\right)\right\|_{L^{1}\left(D_{\delta / 4}, \rho\right)}+\left\|4 \boldsymbol{H}\left(\kappa_{m}\right)-\right\| p_{m}\left\|^{2} \varphi_{\mathrm{JS}}\right\|_{L^{1}\left(K_{m}^{t_{0}}\right)} \\
& \quad \leq 4 c_{1}\left\|\beta_{m}\right\|_{L^{1}\left(D_{\delta / 4}, \rho\right)}+c_{2}\left(1+\left\|p_{m}\right\| \sqrt{t_{0}}\right) \leq c_{3}\left(1+\left\|p_{m}\right\| \sqrt{t_{0}}\right)
\end{aligned}
$$

where $c_{3}$ depends on $\dot{R}^{\prime}$ only. We conclude as in (a). 


\section{Appendix A. The collar lemma and a convergence criterion}

Lemma A.1 (Collar lemma, [Kee74], [Mat76]). For every simple closed geodesic $\gamma \subset$ $\Sigma$ in a hyperbolic surface and for every "side" of $\gamma$, there exists an embedded hypercycle $\gamma^{\prime}$ parallel to $\gamma$ (on the prescribed side) such that the area of the annulus enclosed by $\gamma$ and $\gamma^{\prime}$ is $\ell /(2 \sinh (\ell / 2))$. For $\ell=0$, the geodesic $\gamma$ must be understood to be a cusp and $\gamma^{\prime}$ a horocycle of length 1 . Furthermore, all such annuli (corresponding to distinct geodesics and sides) are disjoint.

We recall here a useful criterion of convergence in $\overline{\mathcal{T}}(S)$.

Proposition A.2 ([Mon09a]). Let $\left\{\left[f_{m}: S \rightarrow\left(\Sigma_{m}, g_{m}\right)\right]\right\}$ be a sequence of points in $\mathcal{T}(S)$ and let $[f: S \rightarrow(\Sigma, g)] \in \overline{\mathcal{T}}(S)$. The following are equivalent:

(a) $\left[f_{m}\right] \rightarrow[f]$ in $\overline{\mathcal{T}}(S)$,

(b) there exists $\tilde{f}_{m} \in\left[f_{m}\right]$ such that the metrics $\left(\tilde{f}_{m} \circ f^{-1}\right)^{*}\left(g_{m}\right) \rightarrow g$ uniformly on compact subsets of the smooth locus of $\Sigma$,

(c) there exists $\tilde{f}_{m} \in\left[f_{m}\right]$ such that the conformal distorsion $K\left(\tilde{f}_{m} \circ f^{-1}\right) \rightarrow 1$ uniformly on compact subsets of the smooth locus of $\Sigma$.

\section{Appendix B. The continuity of $\widehat{W}$}

The following proof shares some ideas with $[\mathrm{ACGH}]$ (to which we refer for a more detailed discussion of the case with $n$ cusps). The bijectivity of $\widehat{\boldsymbol{W}}$ is a direct consequence of the work of Bowditch-Epstein, Penner and Luo. We begin with some preparatory observations.

Definition B.1. Let $([f: S \rightarrow \Sigma], p) \in \widehat{\mathcal{T}}(S)(0)$ be a projectively decorated surface and let $B_{i} \subset \Sigma$ be the embedded horoball at $x_{i}=f\left(C_{i}\right)$ with radius $p_{i}$. The associated truncated surface is $\Sigma^{\mathrm{tr}}:=\Sigma \backslash\left(B_{1} \cup \cdots \cup B_{n}\right)$, the reduced length of an arc $\alpha \in \mathcal{A}(S)$ at $f$ is $\tilde{\ell}_{\alpha}(f):=\ell\left(\Sigma^{\operatorname{tr}} \cap f(\alpha)\right)$, and the $\lambda$-length of $\alpha$ is $\lambda_{\alpha}(f):=\exp \left(\tilde{\ell}_{\alpha}(f) / 2\right)$.

We remark that our definition of $\lambda$-length differs from Penner's original one ([Pen87]) by a factor of $\sqrt{2}$.

Lemma B.2. Let $\left\{\left[f_{m}: S \rightarrow \Sigma_{m}\right]\right\} \subset \mathcal{T}(S)$ be a sequence that converges to $[f: S \rightarrow$ $\Sigma] \in \widehat{\mathcal{T}}^{\mathrm{vis}}(S)$.

(a) Assume $\|\mathcal{L}(f)\|>0$ and let $\mathcal{A}(S)=\mathcal{A}_{\text {fin }} \sqcup \mathcal{A}_{\infty}$, where $\mathcal{A}_{\infty}$ is the subset of arcs $\beta$ such that $\ell_{\beta}(f)=\infty$. Then $\ell_{\alpha}\left(f_{m}\right) / \ell_{\alpha}(f) \rightarrow 1$ uniformly for all $\alpha \in \mathcal{A}_{\text {fin }}$. Moreover, if $\mathcal{A}_{\infty} \neq \emptyset$, then there exists a diverging sequence $\left\{L_{m}\right\} \subset \mathbb{R}_{+}$such that $\ell_{\beta}\left(f_{m}\right) \geq L_{m}$ for all $\beta \in \mathcal{A}_{\infty}$. Hence, $t_{\bullet}\left(f_{m}\right) \rightarrow t_{\bullet}(f)$ uniformly.

(b) Assume $\overline{\mathcal{L}}(f)=(\underline{\tilde{p}}, 0) \in \Delta^{n-1} \times\{0\}$ and let $\mathcal{A}(S)=\mathcal{A}_{\text {fin }} \sqcup \mathcal{A}_{\infty}$, where $\mathcal{A}_{\infty}$ is the subset of arcs $\beta$ such that $\tilde{\ell}_{\beta}(f)=\infty$. Then $\tilde{\ell}_{\alpha}\left(f_{m}\right) / \tilde{\ell}_{\alpha}(f) \rightarrow 1$ uniformly for all $\alpha \in \mathcal{A}_{\text {fin. }}$ Moreover, if $\mathcal{A}_{\infty} \neq \emptyset$, then there exists a diverging sequence $\left\{\tilde{L}_{m}\right\} \subset \mathbb{R}_{+}$ such that $\tilde{\ell}_{\beta}\left(f_{m}\right) \geq \tilde{L}_{m}$ for all $\beta \in \mathcal{A}_{\infty}$. Hence, $\left[t_{\bullet}\left(f_{m}\right)\right] \rightarrow\left[t_{\bullet}(f)\right]$. 
Remark B.3. Here we collect a few facts about how a geodesic boundary component degenerates to a cusp and we set some notation, which will be useful in the proofs of Lemma B.2 b) and in Lemma B.4.

(i) A simple computation shows that a loop at distance $d$ from a closed geodesic of length $\ell$ (also called a "hypercycle") has length $\ell \cosh (d)$.

(ii) For the purposes of this remark, we will assume that $\mathcal{L}\left(f_{m}\right) \rightarrow 0$ and also that $\left\|\mathcal{L}\left(f_{m}\right)\right\| \leq 1$. So we can define $\vartheta_{m} \in[0, \pi / 2]$ by $\sin \left(\vartheta_{m}\right):=\left\|\mathcal{L}\left(f_{m}\right)\right\|$.

(iii) For each boundary circle $C_{i, m}$ of $\Sigma_{m}$, let $H C_{i, m} \subset \Sigma_{m}$ be the hypercycle parallel to $C_{i, m}$ at distance $d_{m}=-\log \tan \left(\vartheta_{m} / 2\right)$ (i.e. $\cosh \left(d_{m}\right)=1 / \sin \left(\vartheta_{m}\right)$ ), which has length $\tilde{p}_{i}\left(f_{m}\right):=p_{i}\left(f_{m}\right) / \sin \left(\vartheta_{m}\right) \leq 1$ and so is embedded in $\Sigma_{m}$.

(iv) Notice that the spine of $\Sigma_{m}$ coincides with the spine of its subsurface $\Sigma_{m}^{\mathrm{tr}}$ obtained by removing the hyperballs bounded by the $H C_{i, m}$ 's; in fact, every geodesic that meets $C_{i, m}$ orthogonally also intersects $H C_{i, m}$ orthogonally.

(v) For every arc $\alpha$, define the reduced length of $\alpha$ at $f_{m}$ to be $\tilde{\ell}_{\alpha}\left(f_{m}\right):=\ell_{\alpha}\left(f_{m}\right)-2 d_{m}$, that is, the length of $f_{m}(\alpha) \cap \Sigma_{m}^{\mathrm{tr}}$. We can define the $\lambda$-length of $\alpha$ as $\lambda_{\alpha}\left(f_{m}\right):=$ $\exp \left(\tilde{\ell}_{\alpha} / 2\right)$.

(vi) Because $H C_{i, m}$ limits to a horocycle of length $\tilde{p}_{i}=\tilde{p}_{i}(f)$ as $m \rightarrow \infty$, we obtain $\lambda_{\alpha}\left(f_{m}\right) \rightarrow \lambda_{\alpha}(f, \underline{p})$.

In the following proof, we will denote by $S_{+}$the open subsurface $f^{-1}\left(\Sigma_{+}\right)$and by $S_{+}^{\mathrm{tr}}$ the preimage under $f$ of the truncated surface $\Sigma_{+}^{\mathrm{tr}}$.

Proof of Lemma B.2. By Proposition A.2 we can assume that $f_{m}^{*}\left(g_{m}\right) \rightarrow f^{*}(g)$ uniformly on compact subsets of $S_{+}$.

Case (a): arcs of infinite length. If $\mathcal{A}_{\infty} \neq \emptyset$, then there exist disjoint loops $\gamma_{1}, \ldots, \gamma_{l}$ $\in \mathcal{C}(S)$ corresponding to the nodes of $\Sigma$ such that $c_{m}=\max _{h} \ell_{\gamma_{h}}\left(f_{m}\right) \rightarrow 0$ as $m \rightarrow \infty$. Clearly, $\beta \in \mathcal{A}_{\infty} \Leftrightarrow i\left(\beta, \gamma_{1}+\cdots+\gamma_{l}\right)>0$.

By the collar lemma, $\ell_{\beta}\left(f_{m}\right)>L_{m}:=T\left(c_{m}\right) / 2$ and so $t_{\beta}\left(f_{m}\right)<T\left(L_{m}\right) \rightarrow 0$ for all $\beta \in \mathcal{A}_{\infty}$. On the other hand, $\left|\ell_{\alpha}\left(f_{m}\right)-\ell_{\alpha}(f)\right| / \ell_{\alpha}(f) \rightarrow 0$ uniformly for all $\alpha \in \mathcal{A}_{\text {fin }}$.

Case (a): arcs of finite length. Fix $\varepsilon>0$. There are finitely many $\operatorname{arcs} \alpha_{1}, \ldots, \alpha_{k} \in$ $\mathcal{A}_{\text {fin }}$ such that $\ell_{\alpha_{i}}(f) \leq T(\varepsilon) /(1-\varepsilon)$ for $i=1, \ldots, k$. Clearly, $\left|t_{\alpha_{i}}\left(f_{m}\right)-t_{\alpha_{i}}(f)\right|<\varepsilon$ for $m$ large. If $\alpha \in \overline{\mathcal{A}}_{\text {fin }}$ and $\alpha \notin\left\{\alpha_{1}, \ldots, \alpha_{k}\right\}$ (that is, $\alpha$ is "long"), then $\ell_{\alpha}\left(f_{m}\right) \geq$ $\ell_{\alpha}(f)(1-\varepsilon)>T(\varepsilon)$ and so $t_{\alpha}\left(f_{m}\right)<\varepsilon$ for $m$ large. Hence, for $\alpha \in \mathcal{A}_{\text {fin }} \backslash\left\{\alpha_{1}, \ldots, \alpha_{k}\right\}$ too, $\left|t_{\alpha}\left(f_{m}\right)-t_{\alpha}(f)\right|<\varepsilon$ for $m$ large.

Case (b): arcs of infinite reduced length. Let $\gamma_{1}, \ldots, \gamma_{l}$ be the curves in the interior of $S$ that are shrunk to nodes of $\Sigma$ and let $J=\left\{j \mid \tilde{p}_{j}=0\right\}$. We can assume that $p_{i}\left(f_{m}\right)<$ $\tilde{p}_{i}(f)<1$ if $i \notin J$ and that $p_{j}\left(f_{m}\right)<1$ if $j \in J$.

Let $c_{m}=\max \left\{\ell_{\gamma_{h}}\left(f_{m}\right)\right\}$ and $c_{m}^{\prime}=\max \left\{p_{j} \mid j \in J\right\}$. Clearly, if $\beta \in \mathcal{A}_{\infty}$ intersects some $\gamma_{h}$, then $\tilde{\ell}_{\beta}\left(f_{m}\right) \geq T\left(c_{m}\right) / 2 \rightarrow \infty$. If $\beta \in \mathcal{A}_{\infty}$ does not intersect any $\gamma_{h}$, then it meets some $C_{j}$ with $j \in J$. Because of the collar lemma, there is a hypercycle embedded in $\Sigma_{m}$ at distance $\delta_{j, m}$ from $f_{m}\left(C_{j}\right)$, with $p_{j}\left(f_{m}\right) \cosh \left(\delta_{j, m}\right)=1$. As $p_{j}\left(f_{m}\right) \cosh \left(d_{m}\right)=$ $p_{j}\left(f_{m}\right) / \sin \left(\vartheta_{m}\right)$, we get $\cosh \left(\delta_{j, m}\right) / \cosh \left(d_{m}\right)=\sin \left(\vartheta_{m}\right) / p_{j}\left(f_{m}\right)$ and so $\delta_{j, m}-d_{m} \approx$ $2 \log \left(\sin \left(\vartheta_{m}\right) / p_{j}\left(f_{m}\right)\right) \geq 2 \log \left(\sin \left(\vartheta_{m}\right) / c_{m}^{\prime}\right) \rightarrow \infty$ for $j \in J$. Hence, $\tilde{\ell}_{\beta}\left(f_{m}\right) \geq \tilde{L}_{m}:=$ $\min \left\{T\left(c_{m}\right) / 2,2 \log \left(\sin \left(\vartheta_{m}\right) / c_{m}^{\prime}\right)\right\} \rightarrow \infty$. 
Case (b): arcs of finite reduced length. The uniform convergence of $f_{m}^{*}\left(g_{m}\right)$ on the compact subsets of $S_{+}^{\operatorname{tr}}$ ensures that $\left|\tilde{\ell}_{\alpha}\left(f_{m}\right)-\tilde{\ell}_{\alpha}(f)\right| / \tilde{\ell}_{\alpha}(f) \rightarrow 0$ uniformly for all $\alpha \in$ $\mathcal{A}_{\text {fin. }}$.

Let $\alpha_{0} \in \mathcal{A}_{\text {fin }}$ be the arc with smallest $\tilde{\ell}_{\alpha_{0}}(f)$. Fix $\varepsilon>0$. There are finitely many arcs $\alpha_{1}, \ldots, \alpha_{k} \in \mathcal{A}_{\text {fin }}$ such that $\tilde{\ell}_{\alpha_{i}}(f) \leq \tilde{\ell}_{\alpha_{0}}(f)-2 \log (\varepsilon)$ for $i=1, \ldots, k$. Clearly,

$$
\frac{t_{\alpha_{i}}\left(f_{m}\right)}{t_{\alpha_{0}}\left(f_{m}\right)} \rightarrow \frac{t_{\alpha_{i}}(f)}{t_{\alpha_{0}}(f)}=\frac{\lambda_{\alpha_{0}}(f)}{\lambda_{\alpha_{i}}(f)} \quad \text { for } i=1, \ldots, k \text {. }
$$

If $\alpha \in \mathcal{A}_{\text {fin }}$ and $\alpha \notin\left\{\alpha_{1}, \ldots, \alpha_{k}\right\}$ (and so $\alpha$ is "long"), then

$$
\frac{t_{\alpha}\left(f_{m}\right)}{t_{\alpha_{0}}\left(f_{m}\right)} \leq \varepsilon+\sqrt{\exp \left[\tilde{\ell}_{\alpha_{0}}\left(f_{m}\right)-\tilde{\ell}_{\alpha}\left(f_{m}\right)\right]}<2 \varepsilon
$$

for $m$ large. Hence, $\left|t_{\alpha}\left(f_{m}\right) / t_{\alpha_{0}}(f)\right|<2 \varepsilon$ for $m$ large and $\alpha \in \mathcal{A}_{\text {fin }} \backslash\left\{\alpha_{1}, \ldots, \alpha_{k}\right\}$.

Lemma B.4. $\widehat{W}$ is continuous.

Notice that $\widehat{\mathcal{T}}^{\mathrm{vis}}(S)$ and $|\mathfrak{A}(S)| \times[0, \infty)$ have countable systems of neighbourhoods at each point. As $\mathcal{T}(S)$ is dense in $\widehat{\mathcal{T}}^{\text {vis }}(S)$, in order to test the continuity of $\widehat{\boldsymbol{W}}$, we can consider a sequence $\left\{\left[f_{m}: S \rightarrow \Sigma_{m}\right]\right\} \subset \mathcal{T}(S)$ converging to a point $[f: S \rightarrow \Sigma] \in$ $\widehat{\mathcal{T}}^{\text {vis }}(S)$. Moreover, because of Proposition A.2. we can assume that $f_{m}^{*}\left(g_{m}\right) \rightarrow f^{*}(g)$ uniformly on the compact subsets of $S_{+}$.

Assume first that $[f] \notin \widehat{\mathcal{T}}^{\mathrm{vis}}(S)(0)$. We break the proof into four steps:

(1) "stabilization" of the spine, that is, $f^{-1}\left(\boldsymbol{A}_{\mathrm{sp}}(\Sigma)\right) \subseteq f_{m}^{-1}\left(\boldsymbol{A}_{\mathrm{sp}}\left(\Sigma_{m}\right)\right)$ for $m$ large,

(2) splitting the arc systems $\boldsymbol{A}_{\mathrm{sp}}\left(\Sigma_{m}\right)$ into subsystems of long arcs and finite arcs,

(3) convergence of the widths of long arcs,

(4) convergence of the width of finite arcs.

In step (4), $w_{\mathrm{sp}}\left(\vec{\alpha}, f_{m}\right)$ is computed by applying $(*)$ to a hexagon $H_{m}$ of $S \backslash f_{m}^{-1}\left(\boldsymbol{A}_{\mathrm{sp}}\left(\Sigma_{m}\right)\right)$ that bounds $\vec{\alpha}$. There are three cases:

(4a) $H_{m}$ is bordered by three arcs of bounded length,

(4b) $H_{m}$ is bordered by two arcs of bounded length and one divergent arc,

(4c) $H_{m}$ is bordered by one arc of bounded length and one divergent arc.

We show that (4b) does not occur and that one has the desired limit in (4a) and (4c).

The case of $[f] \in \widehat{\mathcal{T}}^{\mathrm{vis}}(S)(0)$ is similar, using reduced lengths and normalized widths.

Proof. Suppose $[f] \notin \widehat{\mathcal{T}}^{\mathrm{vis}}(S)(0)$.

The distance function $d_{f_{m}}\left(-, \partial S_{+}\right): S_{+} \rightarrow \mathbb{R}_{+}$with respect to the metric $f_{m}^{*} g_{m}$ converges uniformly on compact subsets of $S_{+}$.

Step 1: stability of the spine. Let $\mathcal{E}$ be the set of edges of $\operatorname{Sp}(\Sigma)$ and let $m_{i}$ be the midpoint of the edge $E_{i} \in \mathcal{E}$ in $\Sigma$. Let $\gamma_{i, 1}$ and $\gamma_{i, 2}$ be the shortest geodesics that join $m_{i}$ to $\partial \Sigma$ and $\alpha_{i}:=f^{-1}\left(\gamma_{i, 1}^{-1} * \gamma_{i, 2}\right)$ the associated arc. Let $d\left(m_{i}, \partial \Sigma\right)=\ell\left(\gamma_{E, 1}\right)=\ell\left(\gamma_{E, 2}\right)$ and write $d^{\prime}\left(m_{i}, \partial \Sigma\right)$ for the minimum length of a geodesic that joins $m_{i}$ to $\partial \Sigma$ and is not homotopic to $\gamma_{i, 1}$ or $\gamma_{i, 2}$. Finally, set $\varepsilon=\min \left\{d^{\prime}\left(m_{i}, \partial \Sigma\right)-d\left(m_{i}, \partial \Sigma\right) \mid E_{i} \in \mathcal{E}\right\}>0$. 
Because $d_{f_{m}}\left(f^{-1}\left(m_{i}\right), \partial S_{+}\right)$and $d_{f_{m}}^{\prime}\left(f^{-1}\left(m_{i}\right), \partial S_{+}\right)$also converge as $m \rightarrow \infty$, their difference is eventually positive and so the arc $\alpha_{i}$ is still dual to some edge of the spines $f_{m}^{-1}\left(\operatorname{Sp}\left(\Sigma_{m}\right)\right)$ of $\left(S, f_{m}^{*}\left(g_{m}\right)\right)$ for $m$ large. Thus, up to discarding finitely many terms of the sequence, we can assume that $\boldsymbol{A}:=f^{-1}\left(\boldsymbol{A}_{\mathrm{sp}}(\Sigma)\right) \subseteq \boldsymbol{A}_{m}:=f_{m}^{-1}\left(\boldsymbol{A}_{\mathrm{sp}}\left(\Sigma_{m}\right)\right)$.

Step 2: long and finite arc subsystems. The set of long arcs $\mathcal{A}_{\infty}$ is the set of all the arcs that are not supported in $S_{+}$. The set of $\boldsymbol{A}$-compatible finite arcs $\boldsymbol{A}^{\text {fin }}$ consists of those $\operatorname{arcs} \alpha$ supported in $S_{+}$such that $\boldsymbol{A} \cup\{\alpha\}$ is still a system of arcs. Notice that $\boldsymbol{A} \subseteq \boldsymbol{A}^{\text {fin }}$ and that $\boldsymbol{A}^{\text {fin }}$ is finite.

Because of Theorem 3.9 we can assume in our computation that $\boldsymbol{A}_{m}:=$ $f_{m}^{-1}\left(\boldsymbol{A}_{\mathrm{sp}}\left(\Sigma_{m}\right)\right)$ is maximal. Moreover, we split $\boldsymbol{A}_{m}$ as the union of the subsystems $\boldsymbol{A}_{m}^{\text {fin }}:=\boldsymbol{A}_{m} \cap \boldsymbol{A}^{\text {fin }}$ and $\boldsymbol{A}_{m} \cap \mathcal{A}_{\infty}$.

Step 3: widths of long arcs. By Lemma B.2(a), $\ell_{\beta}\left(f_{m}\right) \geq L_{m}$ equidiverge and so $w_{\boldsymbol{A}_{m}}\left(\vec{\beta}, f_{m}\right) \leq t_{\beta}\left(f_{m}\right) / 2 \leq T\left(L_{m}\right) / 2$ for all $\beta \in \boldsymbol{A}_{m} \cap \mathcal{A}_{\infty}$, where $T\left(L_{m}\right) \rightarrow 0$.

Step 4: widths of finite arcs. It is sufficient to show the following: for every $\alpha \in$ $\boldsymbol{A}^{\mathrm{fin}}$ and for every subsequence $\left\{f_{m_{i}}\right\}$ such that $\alpha \in \boldsymbol{A}_{m_{i}}$, we have $w_{\boldsymbol{A}_{m_{i}}}\left(\vec{\alpha}, f_{m_{i}}\right) \rightarrow$ $w_{\boldsymbol{A}_{m_{i}}}(\vec{\alpha}, f)$. As $\boldsymbol{A}_{m_{i}}$ is maximal, it decomposes $S$ into hexagons: let $H_{i}$ be a hexagon bounded by $\vec{\alpha}$. Up to subsequences, we can assume that we are in either of the following three cases for every $i$.

(4a) $H_{i}$ bounded by three finite arcs. Let $\vec{\alpha}, \vec{\alpha}_{i}^{\prime}, \vec{\alpha}_{i}^{\prime \prime}$ be finite arcs of $\boldsymbol{A}_{m_{i}}$ that bound $H_{i}$. Up to subsequences, we can assume that $\vec{\alpha}_{i}^{\prime}=\vec{\alpha}^{\prime}$ and $\vec{\alpha}_{i}^{\prime \prime}=\vec{\alpha}^{\prime \prime}$. Then

$$
\sinh \left(w_{\boldsymbol{A}_{m_{i}}}\left(\vec{\alpha}, f_{m_{i}}\right)\right)=\frac{s_{\alpha^{\prime}}\left(f_{m_{i}}\right)^{2}+s_{\alpha^{\prime \prime}}\left(f_{m_{i}}\right)^{2}-s_{\alpha}\left(f_{m_{i}}\right)^{2}}{2 s_{\alpha^{\prime}}\left(f_{m_{i}}\right) s_{\alpha^{\prime \prime}}\left(f_{m_{i}}\right) \sqrt{s_{\alpha}\left(f_{m_{i}}\right)^{2}-1}} .
$$

Because $f_{m_{i}}^{*}\left(g_{m_{i}}\right) \rightarrow f^{*}(g)$ uniformly on compact subsets of $S_{+}$, the lengths of the arcs supported in $S_{+}$converge and so $w_{\boldsymbol{A}_{m_{i}}}\left(\vec{\alpha}, f_{m_{i}}\right) \rightarrow w_{\boldsymbol{A}_{m_{i}}}(\vec{\alpha}, f)$.

(4b) $H_{i}$ bounded by two finite arcs. Let $\vec{\alpha}, \vec{\alpha}_{i}^{\prime}, \vec{\beta}_{i}$ be the arcs of $\boldsymbol{A}_{m_{i}}$ that bound $H_{i}$, where $\alpha_{i}^{\prime}$ is finite and $\beta_{i}$ is long. Up to subsequences, we can assume that $\vec{\alpha}_{i}^{\prime}=\vec{\alpha}^{\prime}$ is fixed. The formula

$$
\cosh \left(d\left(\vec{\alpha}, \bar{\alpha}^{\prime}\right)\right)=\frac{\cosh \left(a_{i}\right) \cosh \left(a_{i}^{\prime}\right)+\cosh \left(b_{i}\right)}{\sinh \left(a_{i}\right) \sinh \left(a_{i}^{\prime}\right)}
$$

(where $a_{i}, a_{i}^{\prime}, b_{i}$ are the lengths of $\alpha, \alpha^{\prime}, \beta_{i}$ at $f_{m_{i}}$ ) and the divergence of $b_{i}$ imply that $d\left(\vec{\alpha}, \bar{\alpha}^{\prime}\right)$ diverges, which contradicts the fact that the boundary lengths are bounded. Hence, this case does not occur.

(4c) $H_{i}$ bounded by one finite arc. Let $\vec{\alpha}, \vec{\beta}_{i}, \vec{\beta}_{i}^{\prime}$ be the arcs in $\boldsymbol{A}_{m_{i}}$ that bound $H_{i}$, where $\beta_{i}, \beta_{i}^{\prime}$ are long. Let $x_{\alpha, i}, x_{\beta, i}, x_{\beta^{\prime}, i}$ be the lengths of the edges of $H_{i}$ opposite to $\alpha, \beta_{i}, \beta_{i}^{\prime}$ and let $a_{i}, b_{i}, b_{i}^{\prime}$ be the lengths of $\alpha, \beta_{i}, \beta_{i}^{\prime}$ at $f_{m_{i}}$. Remember that $w_{\boldsymbol{A}_{m_{i}}}\left(\vec{\beta}_{i}, f_{m_{i}}\right), w_{\boldsymbol{A}_{m_{i}}}\left(\vec{\beta}_{i}^{\prime}, f_{m_{i}}\right) \rightarrow 0$, whereas $w_{\boldsymbol{A}_{m_{i}}}\left(\vec{\alpha}, f_{m_{i}}\right)$ is bounded. Notice that $x_{\beta, i}-w_{\boldsymbol{A}_{m_{i}}}\left(\vec{\alpha}, f_{m_{i}}\right)=w_{\boldsymbol{A}_{m_{i}}}\left(\vec{\beta}_{i}, f_{m_{i}}\right) \rightarrow 0$ and $\operatorname{so} \cosh \left(x_{\beta, i}\right)-\cosh \left(w_{\boldsymbol{A}_{m_{i}}}\left(\vec{\alpha}, f_{m_{i}}\right)\right)$ $\rightarrow 0$. The same holds for $\beta_{i}^{\prime}$, thus $\cosh \left(x_{\beta, i}\right)-\cosh \left(x_{\beta^{\prime}, i}\right) \rightarrow 0$. But

$$
\cosh \left(x_{\beta, i}\right)=\frac{\cosh \left(b_{i}^{\prime}\right) \cosh \left(a_{i}\right)+\cosh \left(b_{i}\right)}{\sinh \left(b_{i}^{\prime}\right) \sinh \left(a_{i}\right)}=\frac{1}{\tanh \left(a_{i}\right) \tanh \left(b_{i}^{\prime}\right)}+\frac{\cosh \left(b_{i}\right)}{\sinh \left(a_{i}\right) \sinh \left(b_{i}^{\prime}\right)}
$$


and similarly for $x_{\beta_{i}^{\prime}}$, so that we obtain

$$
\frac{2 \sinh \left(b_{i}-b_{i}^{\prime}\right)}{\sinh \left(a_{i}\right)}=\frac{e^{b_{i}-b_{i}^{\prime}}-e^{b_{i}^{\prime}-b_{i}}}{\sinh \left(a_{i}\right)} \approx \cosh \left(x_{\beta, i}\right)-\cosh \left(x_{\beta^{\prime}, i}\right) \rightarrow 0,
$$

which implies that $\left|b_{i}-b_{i}^{\prime}\right| \rightarrow 0$, because $a_{i} \rightarrow a \in(0, \infty)$.

Consequently,

$$
\cosh \left(w_{\boldsymbol{A}_{m_{i}}}\left(\vec{\alpha}, f_{m_{i}}\right)\right) \rightarrow \frac{1}{\tanh (a)}+\frac{1}{\sinh (a)}=\frac{1}{\tanh (a / 2)},
$$

which gives $w_{\boldsymbol{A}_{m_{i}}}\left(\vec{\alpha}, f_{m_{i}}\right) \rightarrow t_{\alpha}(f) / 2=w_{\boldsymbol{A}}(\vec{\alpha})$.

Suppose now $[f, \underline{p}] \in \widehat{\mathcal{T}}^{\mathrm{vis}}(S)(0)$.

We use the notation in Remark B.3 and we assume as before that $\boldsymbol{A}_{m}$ is maximal.

Notice that

$$
\begin{aligned}
\lambda_{\alpha}\left(f_{m}\right) & =e^{\ell_{\alpha} / 2-d_{m}}=e^{-d_{m}}\left(s_{\alpha}\left(f_{m}\right)+\sqrt{s_{\alpha}\left(f_{m}\right)^{2}-1}\right) \\
& =\tan \left(\vartheta_{m} / 2\right)\left(s_{\alpha}\left(f_{m}\right)+\sqrt{s_{\alpha}\left(f_{m}\right)^{2}-1}\right) \approx \vartheta_{m} s_{\alpha}\left(f_{m}\right)+O\left(\vartheta_{m}^{3} s_{\alpha}\left(f_{m}\right)\right) .
\end{aligned}
$$

In step (4) below, the normalized widths $\tilde{w}_{\boldsymbol{A}_{m}}=2 w_{\boldsymbol{A}_{m}} / \sin \left(\vartheta_{m}\right)$ will be shown to limit to the simplicial coordinates. Since the map $\widehat{\boldsymbol{W}}$ reduces to BEP's map for cusped surfaces, we will also use the term "normalized widths" (instead of "simplicial coordinates") for homogeneity.

As $\|\underline{\tilde{p}}\|=1$, we can assume that $p_{i}\left(f_{m}\right)<\tilde{p}_{i}\left(f_{m}\right)$. The proof follows the same path as before, with some modifications.

Step 1: stability of the spine. By Proposition A.2 (see [Mon09a] for more details), we can assume that $f_{m}\left(S^{\mathrm{tr}}\right)=\Sigma_{m}^{\mathrm{tr}}$ and that the metrics $f_{m}^{*}\left(g_{m}\right)$ converge uniformly on compact subsets of $S_{+}^{\mathrm{tr}}$. Hence, the above argument works by replacing $\Sigma$ and $S$ by their truncations.

Step 2: long and finite arc subsystems. We define long and finite arcs as in the previous case. Because $\underline{p} \rightarrow 0$, the geometry of the cusp implies that $\tilde{w}_{A_{m}}\left(\vec{\alpha}, f_{m}\right) \leq$ $t_{\alpha}\left(f_{m}\right) / \sin \left(\vartheta_{m}\right) \approx 2 \exp \left(-\tilde{\ell}_{\alpha} / 2\right)$ for all $\alpha \in \boldsymbol{A}_{m}$.

Step 3: reduced widths of long arcs. By Lemma B.2(b), $\tilde{\ell}_{\beta}\left(f_{m}\right) \geq \tilde{L}_{m}$ and $\vartheta_{m} s_{\beta}\left(f_{m}\right)$ $\approx \lambda_{\beta}\left(f_{m}\right) \geq \exp \left(\tilde{L}_{m} / 2\right)$ equidiverge and so $\tilde{w}_{\boldsymbol{A}_{m}}\left(\hat{\beta}, f_{m}\right) \leq 2 \exp \left(-\tilde{L}_{m} / 2\right)$ for all $\beta \in$ $\boldsymbol{A}_{m} \cap \mathcal{A}_{\infty}$, where $\exp \left(-\tilde{L}_{m} / 2\right) \rightarrow 0$.

Step 4: reduced widths of finite arcs. For all $\alpha \in A^{\text {fin }}$ we have $\lambda_{\alpha}(f)<\infty$ and $\left|\lambda_{\alpha}\left(f_{m}\right)-\lambda_{\alpha}(f)\right| \rightarrow 0$, and $\left|\lambda_{\alpha}\left(f_{m}\right)-\vartheta_{m} s_{\alpha}\left(f_{m}\right)\right| \rightarrow 0$.

Let $\left(f_{m_{i}}\right)$ be a subsequence with $\alpha \in \boldsymbol{A}_{m_{i}}$ and let $H_{i}$ be a hexagon bounded by $\vec{\alpha}$.

(4a) $H_{i}$ bounded by three finite arcs. Let $\vec{\alpha}, \vec{\alpha}_{i}^{\prime}, \vec{\alpha}_{i}^{\prime \prime}$ be the arcs bounded by $H_{i}$. As before, we can assume that $\vec{\alpha}_{i}^{\prime}=\vec{\alpha}^{\prime}$ and $\vec{\alpha}_{i}^{\prime \prime}=\vec{\alpha}^{\prime \prime}$. Because of the uniform convergence of $f_{m_{i}}^{*}\left(g_{m_{i}}\right) \rightarrow f^{*}(g)$ on compact subsets of $S_{+}^{\mathrm{tr}}$, we have $\tilde{w}_{\boldsymbol{A}_{m_{i}}}\left(\vec{\alpha}, f_{m_{i}}\right) \rightarrow \tilde{w}_{\boldsymbol{A}_{m_{i}}}(\vec{\alpha}, f)$. 
The simplicial coordinate of $\vec{\alpha}$ at $f_{m_{i}}$ is

$$
X_{\boldsymbol{A}_{m_{i}}}\left(\vec{\alpha}, f_{m_{i}}\right)=\frac{\lambda_{\alpha^{\prime}}\left(f_{m_{i}}\right)^{2}+\lambda_{\alpha^{\prime \prime}}\left(f_{m_{i}}\right)^{2}-\lambda_{\alpha}\left(f_{m_{i}}\right)^{2}}{\lambda_{\alpha^{\prime}}\left(f_{m_{i}}\right) \lambda_{\alpha^{\prime \prime}}\left(f_{m_{i}}\right) \lambda_{\alpha}\left(f_{m_{i}}\right)}
$$

It is easy to check that $\tilde{w}_{\boldsymbol{A}_{m_{i}}}\left(\vec{\alpha}, f_{m_{i}}\right) \rightarrow X_{\boldsymbol{A}_{m_{i}}}(\vec{\alpha}, f)$.

(4b) $H_{i}$ bounded by two finite arcs. Let $\vec{\alpha}, \vec{\alpha}_{i}^{\prime}, \vec{\beta}_{i}$ be the arcs bounded by $H_{i}$, with $\beta_{i}$ long. There is a simple closed (nonboundary) curve $\gamma_{i} \subset S$ that is contracted to a node by $f$ and that intersects $\beta_{i}$. Up to subsequences, we can assume that $\vec{\alpha}_{i}^{\prime}=\alpha^{\prime}$ and $\gamma_{i}=\gamma$. Either $\alpha$ or $\alpha^{\prime}$ intersects $\gamma$, which contradicts the fact that $\alpha$ and $\alpha^{\prime}$ are finite arcs.

(4c) $H_{i}$ bounded by one finite arc. Let $\vec{\alpha}, \vec{\beta}_{i}, \vec{\beta}_{i}^{\prime}$ be the arcs bounded by $H_{i}$, where $\beta_{i}$ and $\beta_{i}^{\prime}$ are long. Because $\cosh \left(x_{\alpha, i}\right) \approx 1+x_{\alpha, i}^{2} / 2$ and

$$
\begin{aligned}
\cosh \left(x_{\alpha, i}\right)=\frac{\cosh \left(b_{i}\right) \cosh \left(b_{i}^{\prime}\right)+\cosh \left(a_{i}\right)}{\sinh \left(b_{i}\right) \sinh \left(b_{i}^{\prime}\right)} \approx & +2 \exp \left(a_{i}-b_{i}-b_{i}^{\prime}\right) \\
& +2 \exp \left(-2 b_{i}\right)+2 \exp \left(-2 b_{i}^{\prime}\right)
\end{aligned}
$$

we obtain $x_{\alpha, i}^{2} / \vartheta_{m_{i}}^{2} \approx \exp \left(\tilde{a}_{i}-\tilde{b}_{i}-\tilde{b}_{i}^{\prime}\right)+O\left(\vartheta_{m_{i}}^{2}\right) \rightarrow 0$.

Remember that $\tilde{w}_{\boldsymbol{A}_{m_{i}}}\left(\vec{\beta}_{i}, f_{m_{i}}\right), \tilde{w}_{\boldsymbol{A}_{m_{i}}}\left(\vec{\beta}_{i}^{\prime}, f_{m_{i}}\right) \rightarrow 0$ and that $\tilde{w}_{\boldsymbol{A}_{m_{i}}}\left(\vec{\alpha}, f_{m_{i}}\right)$ is bounded. So $x_{\beta, i} / \sin \left(\vartheta_{m_{i}}\right)$ and $x_{\beta^{\prime}, i} / \sin \left(\vartheta_{m_{i}}\right)$ are bounded too.

On the other hand, $\cosh \left(x_{\beta^{\prime}, i}\right) \approx 1+2 \exp \left(b_{i}^{\prime}-b_{i}-a_{i}\right)+2 \exp \left(-2 a_{i}\right)+2 \exp \left(-2 b_{i}\right)$ and so $x_{\beta^{\prime}, i}^{2} \approx 4 \exp \left(b_{i}^{\prime}-b_{i}-a_{i}\right)+4 \exp \left(-2 b_{i}\right)+4 \exp \left(-2 a_{i}\right)$ and $x_{\beta^{\prime}, i}^{2} / \sin ^{2}\left(\vartheta_{m_{i}}\right) \approx$ $\exp \left(\tilde{b}_{i}^{\prime}-\tilde{b}_{i}-\tilde{a}_{i}\right)+O\left(\vartheta_{m_{i}}^{2}\right)$.

Finally,

$$
\tilde{w}_{\boldsymbol{A}_{m_{i}}}\left(\vec{\alpha}, f_{m_{i}}\right) \approx \frac{2 x_{\beta, i}}{\sin \left(\vartheta_{m_{i}}\right)} \approx 2 \exp \left(\frac{\tilde{b}_{i}^{\prime}-\tilde{b}_{i}-\tilde{a}_{i}}{2}\right)+O\left(\vartheta_{m_{i}}^{2}\right)
$$

and an analogous estimate holds with the roles of $\tilde{b}_{i}$ and $\tilde{b}_{i}^{\prime}$ switched. This implies that $\left|\tilde{b}_{i}^{\prime}-\tilde{b}_{i}\right| \rightarrow 0$.

As a consequence, $\tilde{w}_{A_{m_{i}}}\left(\vec{\alpha}, f_{m_{i}}\right) \approx 2 \exp \left(-\tilde{a}_{i} / 2\right) \rightarrow 2 \exp (-\tilde{a} / 2)=2 / \lambda_{\alpha}(f)=$ $X_{\boldsymbol{A}_{m_{i}}}(\vec{\alpha}, f)$.

\section{Appendix C. Two topological remarks}

The following topological lemma will be useful in the proofs of Theorems 3.16, 3.17 and 5.4

Lemma C.1. Let $f: X \rightarrow Y$ be a $G$-equivariant continuous map between metric spaces on which the discrete group $G$ acts by isometries. Assume that the G-orbits on $Y$ are discrete.

(a) If $X / G$ is compact and $\operatorname{stab}(x) \subseteq \operatorname{stab}(f(x))$ has finite index for all $x \in X$, then $f$ is proper. 
(b) If $f$ is injective and the induced map $\bar{f}: X / G \rightarrow Y / G$ is a homeomorphism, then $f$ is a homeomorphism.

Proof. For (a), let $\left\{x_{m}\right\} \subset X$ be a divergent sequence. Up to extracting a subsequence, we can assume that $\left[x_{m}\right] \rightarrow[x] \in X / G$. Thus there exists $g_{m} \in G$ such that $x_{m} \cdot g_{m} \rightarrow x$, that is, $d_{X}\left(x_{m}, x \cdot g_{m}^{-1}\right) \rightarrow 0$. As $\left\{x_{m}\right\}$ is divergent, the sequence $\left\{\left[g_{m}\right]\right\} \subset G / \operatorname{stab}(x)$ is divergent too, and so it is in $G / \operatorname{stab}(f(x))$. Hence, every point of the orbit $f(x) \cdot G$ occurs finitely many times in $\left\{f(x) \cdot g_{m}^{-1}\right\}$, because $f(x) \cdot G$ is discrete. As $f\left(x_{m} \cdot g_{m}\right) \rightarrow f(x)$, we have $d_{Y}\left(f\left(x_{m}\right), f(x) \cdot g_{m}^{-1}\right) \rightarrow 0$ and $\left\{f\left(x_{m}\right)\right\}$ is divergent too.

For (b), let us show first that $f$ is surjective. Because $\bar{f}$ is bijective, for every $y \in Y$ there exists a unique $[x] \in X / G$ such that $\bar{f}([x])=[y]$. Hence, $f(x)=y \cdot g$ for some $g \in G$ and so $f\left(x \cdot g^{-1}\right)=y$.

The injectivity of $f$ also implies that $\operatorname{stab}(x)=\operatorname{stab}(f(x))$ for all $x \in X$.

To prove that $f^{-1}$ is continuous, let $\left\{x_{m}\right\} \subset X$ be a sequence such that $f\left(x_{m}\right) \rightarrow f(x)$ for some $x \in X$. Clearly, $\left[f\left(x_{m}\right)\right] \rightarrow[f(x)]$ in $Y / G$ and so $\left[x_{m}\right] \rightarrow[x]$ in $X / G$, because $\bar{f}$ is a homeomorphism.

Hence, there exists $g_{m} \in G$ such that $z_{m}:=x_{m} \cdot g_{m}^{-1} \rightarrow x$. By continuity of $f$, we have $f\left(z_{m}\right) \rightarrow f(x)$ and by hypothesis $f\left(z_{m}\right) \cdot g_{m} \rightarrow f(x)$. Moreover, $d_{Y}\left(f(x) \cdot g_{m}, f(x)\right) \leq d_{Y}\left(f(x) \cdot g_{m}, f\left(x_{m}\right)\right)+d_{Y}\left(f\left(x_{m}\right), f(x)\right)=d_{Y}\left(f(x), f\left(z_{m}\right)\right)+$ $d_{Y}\left(f\left(x_{m}\right), f(x)\right) \rightarrow 0$ and so $f(x) \cdot g_{m} \rightarrow f(x)$. Hence, $g_{m} \in \operatorname{stab}(f(x))=\operatorname{stab}(x)$ for large $m$, because $G$ acts with discrete orbits on $Y$. As a consequence, for $m$ large enough $d_{X}\left(x_{m}, x\right)=d_{X}\left(z_{m}, x\right) \rightarrow 0$ and so $x_{m} \rightarrow x$ and $f^{-1}$ is continuous at $f(x)$.

Notice that, in order to check that the $G$-orbits on $Y$ are discrete, it is sufficient to show the following:

(•) whenever $y \cdot g_{m} \rightarrow y$ for a certain $y \in Y$ and $\left\{g_{m}\right\} \subset G$, the sequence $\left\{g_{m}\right\}$ eventually lies in $\operatorname{stab}_{G}(y)$.

In fact, because of $(\bullet)$, there is an $\varepsilon>0$ such that $B(y, \varepsilon) \cap y \cdot G=\{y\}$. Given a sequence $\left\{g_{m}\right\} \subset G$ with $z \cdot g_{m} \rightarrow y \in Y$, we have $d\left(y \cdot g_{j}^{-1} g_{i}, y\right) \leq d\left(y \cdot g_{j}^{-1}, z\right)+d\left(z, y \cdot g_{i}^{-1}\right)$ $=d\left(y, z \cdot g_{j}\right)+d\left(z \cdot g_{i}, y\right)<\varepsilon$ for $i, j \geq N_{\varepsilon}$. Thus, $g_{j}^{-1} g_{i} \in \operatorname{stab}_{G}(y)$ and $d\left(z \cdot g_{j}, y\right)=$ $d\left(z \cdot g_{j} g_{j}^{-1} g_{i}, y\right)=d\left(z \cdot g_{i}, y\right)$ for all $i, j \geq N_{\varepsilon}$. Hence, $z \cdot g_{i}=y$ for all $i \geq N_{\varepsilon}$ and so the orbit $z \cdot G$ is discrete.

Another simple topological statement will be occasionally useful.

Lemma C.2. Let $f: X \rightarrow Y$ be a map of metric spaces and let $U \subset X$ be a dense subspace. Assume that

(a) $\left.f\right|_{U}$ is continuous,

(b) for every $\left\{u_{m}\right\} \subset U$ such that $u_{m} \rightarrow x \in X$, we have $f\left(u_{m}\right) \rightarrow f(x)$.

Then $f$ is continuous.

Proof. Let $\left\{x_{m}\right\} \subset X$ be a sequence such that $x_{m} \rightarrow x$. As $U$ is dense in $X$, by (b) we can choose $\left\{u_{m}\right\} \subset U$ such that $d_{X}\left(u_{m}, x_{m}\right)<1 / m$ and $d_{Y}\left(f\left(u_{m}\right), f\left(x_{m}\right)\right)<1 / m$. Then $d_{X}\left(u_{m}, x\right) \rightarrow 0$ and so $d_{Y}\left(f\left(u_{m}\right), f(x)\right) \rightarrow 0$ by (a). Finally, $d_{Y}\left(f\left(x_{m}\right), f(x)\right) \leq$ $d_{Y}\left(f\left(x_{m}\right), f\left(u_{m}\right)\right)+d_{Y}\left(f\left(u_{m}\right), f(x)\right) \rightarrow 0$. 


\section{Appendix D. The continuity of the infinite grafting map}

To prove the continuity of $\left(\mathrm{gr}_{\infty}, \mathcal{L}\right)$ at $q \in \widetilde{\mathcal{T}}(S)$, we split the problem into two distinct cases:

(1) $\mathcal{L}(q)$ bounded and so $q=[f: S \rightarrow \Sigma]$,

(2) $\mathcal{L}(q)$ not bounded and so $q=\tilde{w} \in|\mathfrak{A}(S)|_{\infty}$.

\section{D.1. $\mathcal{L}(q)$ bounded}

Let $\left\{\left[f_{m}: S \rightarrow \Sigma_{m}\right]\right\} \subset \mathcal{T}(S)$ be a sequence that converges to $[f]$, so that $\mathcal{L}\left(f_{m}\right)$ $\rightarrow \mathcal{L}(f)$.

Condition (b) of Proposition A.2 ensures that there are maps $\tilde{f}_{m}: S \rightarrow \Sigma_{m}$ which have a standard behaviour on a neighbourhood of the thin part of $\Sigma_{m}$ and such that the metric $\tilde{f}_{m}^{*}\left(g_{m}\right)$ converges to $f^{*}(g)$ uniformly on the complement (see [Mon09a] for more details). For some fixed $\operatorname{gr}_{\infty}(f): R \rightarrow \operatorname{gr}_{\infty}(\Sigma)$, define $\hat{f}_{m}: R \rightarrow \operatorname{gr}_{\infty}\left(\Sigma_{m}\right)$ in such a way that $F_{m}=\operatorname{gr}_{\infty}(f) \circ \hat{f}_{m}^{-1}: \operatorname{gr}_{\infty}\left(\Sigma_{m}\right) \rightarrow \operatorname{gr}_{\infty}(\Sigma)$ has the following properties.

For every $i$ such that $\ell_{C_{i}}(f)>0$, we denote by $\phi_{m}^{i}: \partial^{i} \Sigma_{m} \rightarrow \partial^{i} \Sigma$ the restriction of $f \circ \tilde{f}_{m}^{-1}$ to the $i$-th boundary component and we put orthonormal coordinates $(x, y)$ (with $y \geq 0$ and $\left.x \in\left[0, \ell_{C_{i}}\right)\right)$ on the $i$-th cylinder $\tilde{C}_{i}$ in such a way that $C_{i}=\{y=0\}$ and $S$ induces on $C_{i}$ the orientation along which $x$ decreases. Then we define $F_{m}(x, y):=$ $\left(\phi_{m}^{i}(x), y\right)$ on the $i$-th cylinder.

For every $j$ such that $\ell_{C_{j}}(f)=0$ and $\ell_{C_{j}}\left(f_{m}\right)>0$, we can assume that $\ell_{C_{j}}\left(f_{m}\right)<$ $1 / 2$ and we can consider a hypercycle $H C_{j} \subset \Sigma_{m} \subset \operatorname{gr}_{\infty}\left(\Sigma_{m}\right)$ parallel to the $j$-th boundary component and of length $2 \ell_{C_{j}}\left(f_{m}\right)$. We define $F_{m}$ to agree with $f \circ \tilde{f}_{m}^{-1}$ on the portion of $\operatorname{gr}_{\infty}\left(\Sigma_{m}\right)$ which is hyperbolic and bounded by the possible hypercycles $H C_{j}$.

Finally, we extend $F_{m}$ outside the possible hypercycles $H C_{j}$ by a diffeomorphism.

Clearly, condition (c) of Proposition A.2 for the sequence $\left\{\hat{f}_{m}\right\}$ and $\operatorname{gr}_{\infty}(f)$ is satisfied and so $\left[f_{m}\right]=\left[\hat{f}_{m}\right] \rightarrow\left[\operatorname{gr}_{\infty}(f)\right]$ in $\widehat{\mathcal{T}}(R, x)$.

\section{D.2. $\mathcal{L}(q)$ not bounded}

Let $S_{+} \subset S$ be the visible subsurface determined (up to isotopy) by $\boldsymbol{A}=\operatorname{supp}(\tilde{w})$.

As $\mathrm{gr}_{\infty}$ is continuous on the $\mathcal{T}(S)$, by Lemma C.2 it is enough to consider a sequence $\left\{\left[f_{m}: S \rightarrow \Sigma_{m}\right]\right\} \subset \mathcal{T}(S)$ that converges to $q=\tilde{w} \in|\mathfrak{A}(S)|_{\infty} \subset \widetilde{\mathcal{T}}(S)$ and such that $\widehat{\boldsymbol{W}}\left(f_{m}\right) \in\left|\boldsymbol{A}_{m}\right|^{\circ} \times \mathbb{R}_{+}$, with $\boldsymbol{A}_{m}$ maximal.

We must show that $\operatorname{gr}_{\infty}\left(f_{m}\right) \rightarrow \operatorname{gr}_{\infty}(q)=[f: R \rightarrow \Sigma]$ in $\overline{\mathcal{T}}(R, x) \times \Delta^{n-1} \times$ $[0, \infty] / \sim$, where $f$ and $\Sigma$ are constructed as in Section 5.2

It will be convenient to denote by $\tilde{w}(\alpha, f)$ the weight $\tilde{w}(\alpha)$ for every $\alpha \in \boldsymbol{A}$. Moreover, we will use the notation $\tilde{w}\left(-, f_{m}\right)$ to denote the normalized quantity $2 w_{\mathrm{sp}}\left(-, f_{m}\right) /\left\|\mathcal{L}\left(f_{m}\right)\right\|$ and $\tilde{w}_{m}(\vec{\alpha}, f)$ to denote $\frac{\tilde{w}\left(\vec{\alpha}, f_{m}\right)}{\tilde{w}\left(\alpha, f_{m}\right)} \tilde{w}(\alpha, f)$. 
Idea of the proof. We will define a sequence of homeomorphisms $\hat{f}_{m}: R \rightarrow$ $\operatorname{gr}_{\infty}\left(\Sigma_{m}\right)$ by explicitly describing $F_{m}=f \circ \hat{f}_{m}^{-1}: \operatorname{gr}_{\infty}\left(\Sigma_{m}\right) \rightarrow \Sigma$. Then we will show that $F_{m}$ is $K_{m}$-quasi-conformal on the compact subsets of the smooth locus of $\dot{\Sigma}_{+}$with $K_{m} \rightarrow 1$. Hence, Proposition A.2 (c) will imply that $\operatorname{gr}_{\infty}\left(f_{m}\right) \rightarrow \operatorname{gr}_{\infty}(q)$.

Let $G_{m}$ be the ribbon graph dual to $\boldsymbol{A}_{m}$ so that $f_{m}\left(G_{m}\right)$ can be identified with $\operatorname{Sp}\left(\Sigma_{m}\right)$. Similarly, let $G$ be the ribbon graph dual to $A$, so that $f(G) \subset \Sigma_{+}$can be identified with the critical graph of $\varphi$.

We can assume that $\boldsymbol{A} \subseteq \boldsymbol{A}_{m}$ for every $m$. One can visualize this inclusion dually as a surjective morphism of ribbon graphs $G_{m} \rightarrow G$ (see [Mon09b], for instance), in which the inverse image of an edge is an edge and the inverse image of a vertex $v$ of $G$ is a subgraph of $G_{m}$ (made of the edges of $G_{m}$ that shrink to $v$ ).

Idea of the construction of $F_{m}$. For every $m$, we subdivide our surfaces $\operatorname{gr}_{\infty}\left(\Sigma_{m}\right)$ and $\Sigma$ into $\hat{R}$-regions (rectangular) and $\hat{V}$-regions (near a vertex), and we coordinatize them. We stress that, even though $\Sigma$ is fixed, some regions and some systems of coordinates on $\Sigma$ may depend on $m$. Then we explicitly define $F_{m}$ on all these regions, except for a small neighbourhood of the vertices of $\operatorname{Sp}\left(\Sigma_{m}\right)=f_{m}\left(G_{m}\right)$ and of the edges in some shrunk subgraph $f_{m}\left(G_{m}(v)\right)$. Where $F_{m}$ is defined, we check that its conformal distortion uniformly converges to 1 . Finally, we extend $F_{m}$ over the remaining part of $\operatorname{gr}_{\infty}\left(\Sigma_{m}\right)$ and we use Proposition A.2 (c) to obtain the desired convergence.

For every $m$ and every small $\varepsilon>0$, define the following regions of $\operatorname{gr}_{\infty}\left(\Sigma_{m}\right)$ and of $\Sigma$.

$\hat{R}$-region on $\operatorname{gr}_{\infty}\left(\Sigma_{m}\right)$ associated to $\alpha \in A_{m}$. Let $\vec{\alpha}$ be an oriented arc on $S$ with support $\alpha \in \boldsymbol{A}_{m}$. Let $P\left(\vec{\alpha}, \Sigma_{m}\right)$ be the projection of the geodesic edge $f_{m}(\alpha)^{*}$ of $\operatorname{Sp}\left(\Sigma_{m}\right)$ to the boundary component of $\Sigma_{m}$ pointed by $f(\vec{\alpha})$ and orient $P\left(\vec{\alpha}, \Sigma_{m}\right)$ coherently with $\vec{\alpha}^{*}$ (and so reversing the orientation induced by $\Sigma_{m}$ ).

Let $R\left(\vec{\alpha}, \Sigma_{m}\right)$ be the geodesic quadrilateral swept out by the shortest geodesics that join $f_{m}\left(\vec{\alpha}^{*}\right)$ and $P\left(\vec{\alpha}, \Sigma_{m}\right)$, and let $\hat{R}\left(\vec{\alpha}, \Sigma_{m}\right)$ be the union of $R\left(\vec{\alpha}, \Sigma_{m}\right)$ and the flat rectangle of $\operatorname{gr}_{\infty}\left(\Sigma_{m}\right)$ of infinite height with base $P\left(\vec{\alpha}, \Sigma_{m}\right)$ (see Figure 6).

$\hat{R}^{\varepsilon}$-region on $\operatorname{gr}_{\infty}\left(\Sigma_{m}\right)$ associated to $\alpha \in A$. Assume now $\alpha \in \boldsymbol{A}$ and let $\left(\vec{\alpha}, \vec{\beta}_{1}, \vec{\beta}_{2}\right)$ bound a hexagon of $S \backslash \boldsymbol{A}_{m}$. The formula

$$
\sinh (a / 2) \sinh (w(\vec{\alpha}))=\frac{s_{\beta_{1}}^{2}+s_{\beta_{2}}^{2}-s_{\alpha}^{2}}{2 s_{\beta_{1}} s_{\beta_{2}}}
$$

shows that $w_{\mathrm{sp}}\left(\vec{\alpha}, f_{m}\right)>0$ for $m$ large enough, because $a\left(f_{m}\right)=\ell_{\alpha}\left(f_{m}\right) \rightarrow 0$. Thus, we can assume that $w_{\mathrm{sp}}\left(\vec{\alpha}, f_{m}\right), w_{\mathrm{sp}}\left(\overleftarrow{\alpha}, f_{m}\right)>0$ for all $\alpha \in \boldsymbol{A}$ and all $m$.

Let $x$ be the arc-length coordinate on $P\left(\vec{\alpha}, \Sigma_{m}\right)$ that is zero at the projection of $s:=$ $\alpha^{*} \cap \alpha$ and let $P_{-}=P \cap\{x \leq 0\}$. Define

$$
R^{\varepsilon}\left(\vec{\alpha}, \Sigma_{m}\right):=\bigcup_{x} r_{x}, \quad \text { where } \quad x \in\left[-(1-\varepsilon) w_{\mathrm{sp}}\left(\vec{\alpha}, f_{m}\right),(1-\varepsilon) w_{\mathrm{sp}}\left(\overleftarrow{\alpha}, f_{m}\right)\right]
$$

where $r_{x}$ is the hypercyclic arc parallel to $f(\alpha)$ that joins $x \in P\left(\vec{\alpha}, \Sigma_{m}\right)$ and $f(\alpha)^{*}$, and let $\hat{R}^{\varepsilon}\left(\vec{\alpha}, \Sigma_{m}\right)$ be the union of $R^{\varepsilon}\left(\vec{\alpha}, \Sigma_{m}\right)$ and the flat rectangle of $\operatorname{gr}_{\infty}\left(\Sigma_{m}\right)$ that leans on it. 


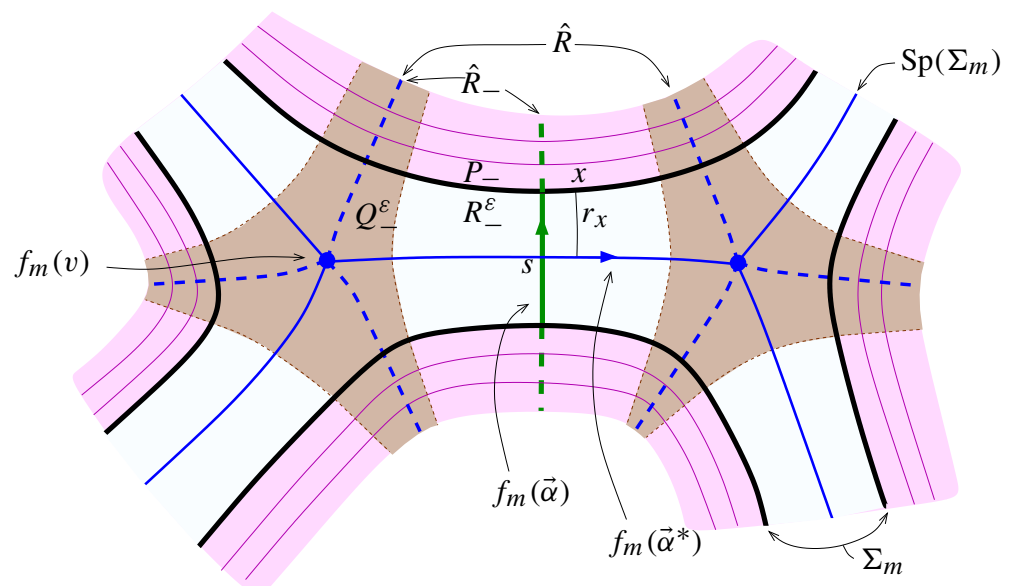

Fig. 6. Regions of $\operatorname{gr}_{\infty}\left(\Sigma_{m}\right)$ associated to $\vec{\alpha}$.

We can put coordinates $(x, y)$ on $R^{\varepsilon}\left(\vec{\alpha}, \Sigma_{m}\right) \cup\left(\hat{R}\left(\vec{\alpha}, \Sigma_{m}\right) \backslash \Sigma_{m}\right)$ such that

- $x$ extends the arc-length coordinate of $P$ and $y$ is compatible with the arc-length coordinate on $\alpha$,

- $(x, y)$ are orthonormal on the flat part $\hat{R}\left(\vec{\alpha}, \Sigma_{m}\right) \backslash \Sigma_{m}$, which corresponds to $\left[-w\left(\vec{\alpha}, f_{m}\right), w\left(\overleftarrow{\alpha}, f_{m}\right)\right] \times[0, \infty)$,

- $(x, y)$ are orthogonal on the hyperbolic part $R^{\varepsilon}\left(\vec{\alpha}, \Sigma_{m}\right)$, which corresponds to [-(1 $\left.\varepsilon) w\left(\vec{\alpha}, f_{m}\right),(1-\varepsilon) w\left(\overleftarrow{\alpha}, f_{m}\right)\right] \times\left[-a\left(f_{m}\right) / 2,0\right]$ in such a way that $\{x=$ const $\}$ is a hypercycle parallel to $f(\alpha)$ and $\{y=$ const $\}$ is a geodesic that crosses $f(\alpha)$ perpendicularly.

Finally, we set $R_{-}^{\varepsilon}:=R^{\varepsilon} \cap\{x \leq 0\}$ and $\hat{R}_{-}^{\varepsilon}:=\hat{R}^{\varepsilon} \cap\{x \leq 0\}$, and we let $\hat{Q}_{-}^{\varepsilon}:=\hat{R}_{-} \backslash \hat{R}_{-}^{\varepsilon}$.

$\hat{R}$ and $\hat{R}^{\varepsilon}$-region on $\Sigma$ associated to $\alpha \in A$. Some of these regions will depend on $m$. First, we denote by $\hat{R}(\vec{\alpha}, \Sigma, m)$ the flat tile $F T_{\vec{\alpha}^{*}} \subset \Sigma$ (see Section 5.2), which comes endowed with coordinates $\tilde{x}=-\tilde{w}_{m}(\vec{\alpha}, f)+\tilde{w}(\alpha, f) u$ (which depends on $m$ ) and $\tilde{y}=$ $\tilde{w}(\alpha, f) v$, so that the Jenkins-Strebel differential $\varphi$ on $\Sigma$ restricts to $(d \tilde{x}+i d \tilde{y})^{2}$ on $\hat{R}(\vec{\alpha}, \Sigma, m)$.

Then, we define $\hat{R}_{-}(\vec{\alpha}, \Sigma, m):=\hat{R}(\vec{\alpha}, \Sigma, m) \cap\{\tilde{x} \leq 0\}$ and $\hat{R}_{-}^{\varepsilon}(\vec{\alpha}, \Sigma, m):=$ $\hat{R}(\vec{\alpha}, \Sigma, m) \cap\left\{-(1-\varepsilon) \tilde{w}_{m}(\vec{\alpha}, f) \leq \tilde{x} \leq 0\right\}$ in such a way that the widths of $\hat{R}_{-}$and $\hat{R}_{-}^{\varepsilon}$ relative to $(\Sigma, m)$ are proportional to the widths of the analogous regions of $\Sigma_{m}$.

Finally we set $\hat{Q}_{-}^{\varepsilon}:=\hat{R}_{-} \backslash \hat{R}_{-}^{\varepsilon}$ and we define similarly the regions with $\tilde{x} \geq 0$.

$\hat{V}^{\varepsilon}$-regions on $\Sigma$. Define $\hat{V}^{\varepsilon}\left(\vec{\beta}_{h}, \Sigma, m\right):=\hat{Q}_{-}^{\varepsilon}\left(\vec{\beta}_{h}, \Sigma, m\right) \cup \hat{Q}_{+}^{\varepsilon}\left(\vec{\beta}_{h+1}, \Sigma, m\right)$ and $\hat{V}^{\varepsilon}(v, \Sigma, m):=\bigcup_{h=1}^{j} \hat{V}^{\varepsilon}\left(\vec{\beta}_{h}, \Sigma, m\right)$. See Figure 7 (right).

The total (normalized) width of $\hat{V}^{\varepsilon}\left(\vec{\beta}_{h}, \Sigma\right)$ is $\tilde{w}_{m}\left(\hat{V}^{\varepsilon}\left(\vec{\beta}_{h}\right), f\right):=\tilde{w}_{m}\left(\vec{\beta}_{h}, f\right)+$ $\tilde{w}_{m}\left(\overleftarrow{\beta}_{h+1}, f\right)$.

$\hat{V}^{\varepsilon}$-region on $\Sigma_{m}$. Let $f\left(\vec{\beta}_{1}\right)^{*}, \ldots, f\left(\vec{\beta}_{j}\right)^{*}$ be the (cyclically ordered) set of edges of $G$ outgoing from a vertex $v$, where $\beta_{h} \in \boldsymbol{A}$ (the indices of the $\beta$ 's are taken in 

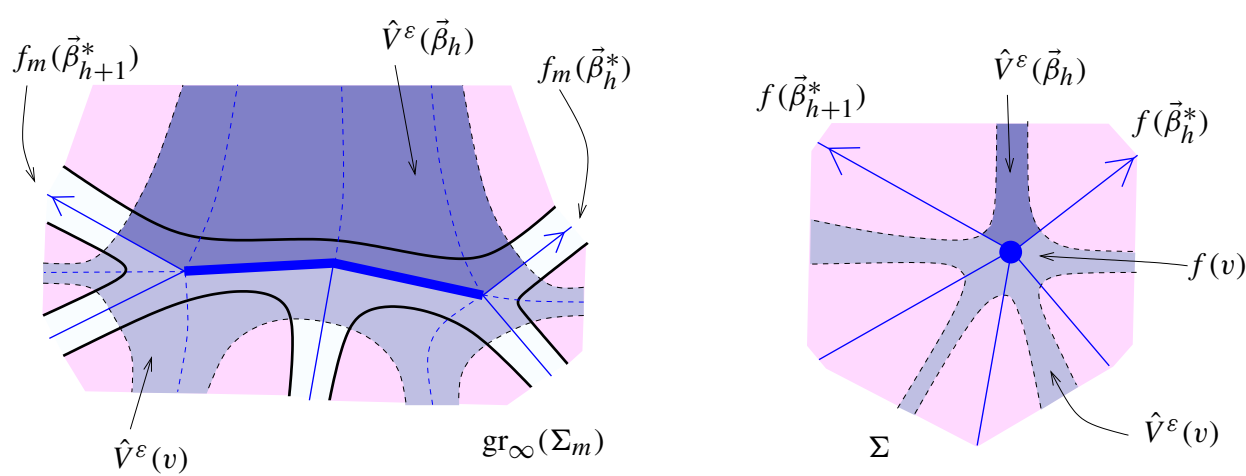

Fig. 7. The thick edges $\gamma_{h, 1}$ and $\gamma_{h, 2}$ are shrunk to the vertex $v$. These $\hat{V}$-regions refer to $\Sigma_{m}$ on the left and to $(\Sigma, m)$ on the right.

$\mathbb{Z} / j \mathbb{Z})$. For every $m$ and $h$ there is an $l_{h} \geq 1$ such that $\vec{\beta}_{h}^{*}, \vec{\gamma}_{h, 1}^{*}=\sigma_{\infty}^{-1}\left(\vec{\beta}_{h}^{*}\right), \vec{\gamma}_{h, 2}^{*}=$ $\sigma_{\infty}^{-2}\left(\vec{\beta}_{h}^{*}\right), \ldots, \vec{\gamma}_{h, l_{h}}^{*}=\sigma_{\infty}^{-l_{h}}\left(\vec{\beta}_{h}^{*}\right)=\bar{\beta}_{h+1}^{*}$ are distinct oriented edges of $G_{m}$ and $\gamma_{h, i} \in$ $\boldsymbol{A}_{m} \backslash \boldsymbol{A}$. Define

$$
\hat{V}^{\varepsilon}\left(\vec{\beta}_{h}, \Sigma_{m}\right):=\hat{Q}_{-}^{\varepsilon}\left(\vec{\beta}_{h}, \Sigma_{m}\right) \cup \hat{Q}_{+}^{\varepsilon}\left(\vec{\beta}_{h+1}, \Sigma_{m}\right) \cup \bigcup_{i=1}^{l_{h}-1} \hat{R}\left(\vec{\gamma}_{h, i}, \Sigma_{m}\right)
$$

(see Figure 7, left) and let

$$
\tilde{w}\left(\hat{V}^{\varepsilon}\left(\vec{\beta}_{h}\right), f_{m}\right)=\varepsilon\left(\tilde{w}\left(\vec{\beta}_{h}, f_{m}\right)+\tilde{w}\left(\widehat{\beta}_{h+1}, f_{m}\right)\right)+\sum_{i=1}^{j_{h}-1} \tilde{w}\left(\gamma_{h, i}, f_{m}\right)
$$

be the total (normalized) width of $\hat{V}^{\varepsilon}\left(\vec{\beta}_{h}, \Sigma_{m}\right)$.

If $v$ is a nonmarked (smooth or singular) vertex of $G$, then we simply set $\hat{V}^{\varepsilon}\left(v, \Sigma_{m}\right)$ $:=\bigcup_{h=1}^{j} \hat{V}^{\varepsilon}\left(\vec{\beta}_{h}, \Sigma_{m}\right)$.

If $f(v)$ is a smooth vertex of $\Sigma$ marked by $x_{i}$, then we set $\hat{V}^{\varepsilon}\left(v, \Sigma_{m}\right):=\left\{x_{i}\right\} \cup \tilde{C}_{i} \cup$ $\bigcup_{h=1}^{j} \hat{V}^{\varepsilon}\left(\vec{\beta}_{h}, \Sigma_{m}\right)$, where $\tilde{C}_{i}$ is the flat cylinder corresponding to $x_{i}$.

Construction of $F_{m}$. We choose $\varepsilon_{m}=\max \left\{1 /\left\|\mathcal{L}\left(f_{m}\right)\right\|, 1-\sum_{\alpha \in A} \tilde{w}\left(\alpha, f_{m}\right)\right\}^{1 / 2}$, so that $\varepsilon_{m} \rightarrow 0, \varepsilon_{m}\left\|\mathcal{L}\left(f_{m}\right)\right\| \rightarrow \infty$ and $\left(1-\sum_{\alpha \in A} \tilde{w}\left(\alpha, f_{m}\right)\right) / \varepsilon_{m} \rightarrow 0$. Moreover, we set $\delta_{m}=\exp \left(-\varepsilon_{m} w\left(\alpha_{0}, f_{m}\right) / 4\right) \rightarrow 0$, where $\alpha_{0} \in \boldsymbol{A}$ and $\tilde{w}\left(\alpha_{0}, f\right)=\min \{\tilde{w}(\alpha, f)>0 \mid$ $\alpha \in \boldsymbol{A}$, so that $a_{i}\left(f_{m}\right)<\delta_{m}$ for $m$ large.

Define $F_{m}: \operatorname{gr}_{\infty}\left(\Sigma_{m}\right) \rightarrow \Sigma$ according to the following prescriptions.

On the $\hat{R}^{\varepsilon_{m}}$-regions. For every $\alpha \in \boldsymbol{A}$ and every orientation $\vec{\alpha}, F_{m}$ continuously maps $\hat{R}_{+}^{\varepsilon_{m}}\left(\vec{\alpha}, \Sigma_{m}\right)$ onto $\hat{R}_{+}^{\varepsilon_{m}}(\vec{\alpha}, \Sigma, m)$ in such a way that

$$
F_{m}(x, y)=\frac{2}{\left\|\mathcal{L}\left(f_{m}\right)\right\|}\left(\frac{\tilde{w}_{m}(\vec{\alpha}, f)}{\tilde{w}\left(\vec{\alpha}, f_{m}\right)} x, y\right) \quad \text { for } y \geq \delta_{m}
$$


and the vertical arcs $\{x\} \times\left[-a / 2, \delta_{m}\right]$ (whose length is $\left.\ell_{x}:=\delta_{m}+a \cosh (x) / 2\right)$ are homothetically mapped to vertical trajectories $\left\{\tilde{x}^{\prime}\right\} \times\left[0,2 \delta_{m} /\left\|\mathcal{L}\left(f_{m}\right)\right\|\right]$. More explicitly, using the $(x, y)$ coordinates on $\operatorname{gr}_{\infty}\left(\Sigma_{m}\right)$ and the $(\tilde{x}, \tilde{y})$ coordinates on $\Sigma$,

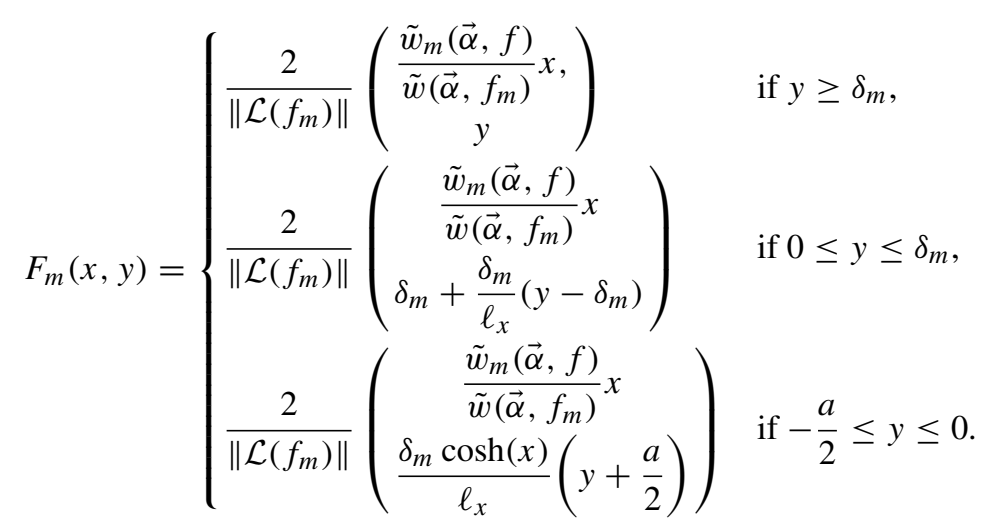

Thus, the differential of $F_{m}$ is

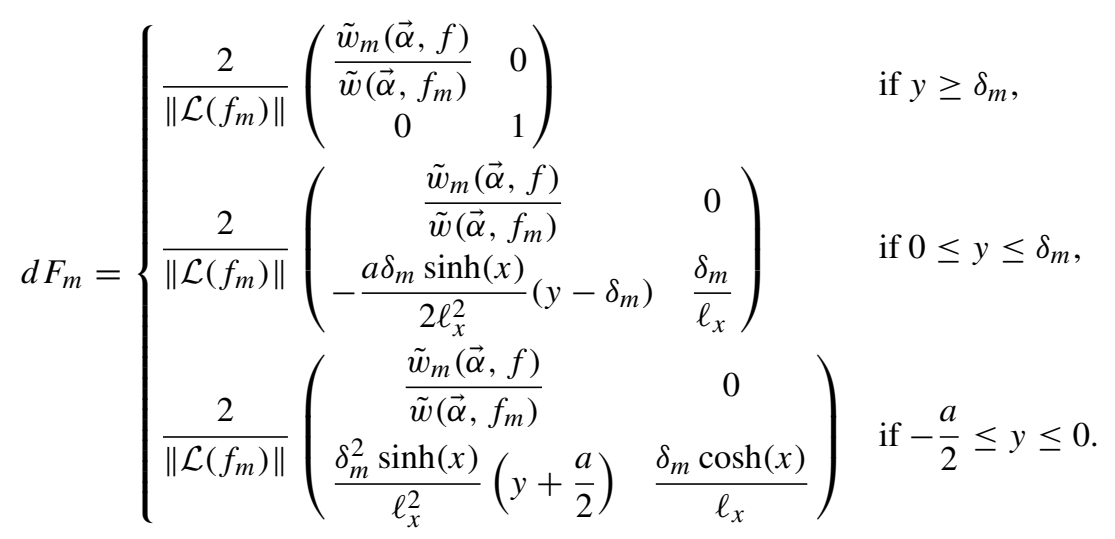

Because the metric $g_{m}$ on $\hat{R}_{+}^{\varepsilon_{m}}\left(\vec{\alpha}, \Sigma_{m}\right)$ in the $x y$-coordinates is

$$
g_{m}= \begin{cases}\left(\begin{array}{ll}
1 & 0 \\
0 & 1
\end{array}\right) & \text { if } y \geq 0 \\
\left(\begin{array}{cc}
1 & 0 \\
0 & \cosh (x)^{2}
\end{array}\right) & \text { if } y \leq 0\end{cases}
$$

we obtain $\left(F_{m}^{-1}\right)^{*}\left(g_{m}\right)=M^{t} M$ (with respect to the $(\tilde{x}, \tilde{y})$ coordinates, which are orthonormal), where 


$$
\begin{aligned}
& M=\sqrt{g_{m}} d F_{m}^{-1}
\end{aligned}
$$

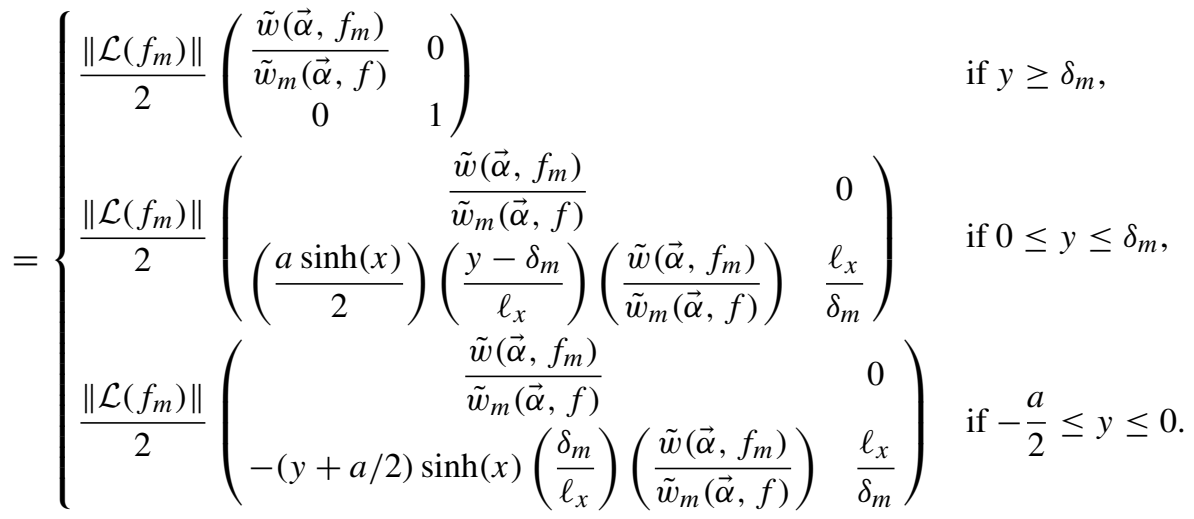

Look at the locus $y \leq \delta_{m}$. Up to switching the orientation, $w\left(\vec{\alpha}, f_{m}\right) \geq w\left(\alpha, f_{m}\right) / 2$ and so $(a / 2) \sinh \left(w\left(\vec{\alpha}, f_{m}\right)\right) \leq 1$. This implies

$$
\frac{a}{2} \sinh (x) \leq \frac{a}{2} \sinh \left[\left(1-\varepsilon_{m}\right) w\left(\vec{\alpha}, f_{m}\right)\right] \lesssim \exp \left(-\varepsilon_{m} w\left(\vec{\alpha}, f_{m}\right)\right),
$$

and similarly $\ell_{x} / \delta_{m}-1 \lesssim \exp \left(-\varepsilon_{m} w\left(\alpha_{0}, f_{m}\right) / 4\right)$. We conclude that, on the $\hat{R}^{\varepsilon_{m}}$-regions, the distortion of $F_{m}$ is bounded by

$$
\max \left\{\frac{\tilde{w}_{m}(\vec{\alpha}, f)}{\tilde{w}\left(\vec{\alpha}, f_{m}\right)}, \frac{\tilde{w}\left(\vec{\alpha}, f_{m}\right)}{\tilde{w}_{m}(\vec{\alpha}, f)} \mid \alpha \in \boldsymbol{A}\right\} \cdot\left(1+\exp \left[-\varepsilon_{m} w\left(\alpha_{0}, f_{m}\right) / 4\right]\right) \rightarrow 1 .
$$

On the $\hat{V}^{\varepsilon_{m}}$-regions away from the vertices. For every oriented edge $\vec{\beta}_{h}^{*}$ of $G$, we require $F_{m}$ to map $\hat{V}^{\varepsilon_{m}}\left(\vec{\beta}_{h}, \Sigma_{m}\right) \cap\left\{y \geq \delta_{m}\right\}$ onto $\hat{V}^{\varepsilon_{m}}\left(\vec{\beta}_{h}, \Sigma, m\right) \cap\left\{\tilde{y} \geq 2 \delta_{m} /\left\|\mathcal{L}\left(f_{m}\right)\right\|\right\}$ as a constant horizontal stretch. In coordinates,

$$
F_{m}=\frac{2}{\left\|\mathcal{L}\left(f_{m}\right)\right\|}\left(\begin{array}{cc}
c & 0 \\
0 & 1
\end{array}\right), \quad \text { where } \quad c=\frac{\tilde{w}_{m}\left(\hat{V}^{\varepsilon_{m}}\left(\vec{\beta}_{h}\right), f\right)}{\tilde{w}\left(\hat{V}^{\varepsilon_{m}}\left(\vec{\beta}_{h}\right), f_{m}\right)}
$$

Notice that the quantity $c-1$ can be rewritten as

$$
\frac{\left(\tilde{w}_{m}\left(\vec{\beta}_{h}, f\right)-\tilde{w}\left(\vec{\beta}_{h}, f_{m}\right)\right)+\left(\tilde{w}_{m}\left(\tilde{\beta}_{h+1}, f\right)-\tilde{w}\left(\tilde{\beta}_{h+1}, f_{m}\right)\right)-\varepsilon_{m}^{-1} \sum_{i} \tilde{w}\left(\gamma_{h, i}, f_{m}\right)}{\tilde{w}\left(\vec{\beta}_{h}, f_{m}\right)+\tilde{w}\left(\tilde{\beta}_{h+1}, f_{m}\right)+\sum_{i} \varepsilon_{m}^{-1} \tilde{w}\left(\gamma_{h, i}, f_{m}\right)}
$$

and so it converges to 0 because $\varepsilon_{m}^{-1} \sum_{i} \tilde{w}\left(\gamma_{i, h}, f_{m}\right) \leq \varepsilon_{m}^{-1}\left(1-\sum_{\alpha \in A} \tilde{w}\left(\alpha, f_{m}\right)\right) \rightarrow 0$. Hence, the distortion of $F_{m}$ converges to 1 .

Extending $F_{m}$ around the vertices of $G$. Let $v$ be a vertex of $G$ and let $\left\{\eta_{i}^{*}\right\}$ be the set of edges of $G_{m}$ shrunk to $v$.

If $f(v) \in f(G) \subset \Sigma$ is smooth, then define $F_{m}$ to be a diffeomorphism between $\hat{V}^{\varepsilon_{m}}\left(v, \Sigma_{m}\right) \backslash\left\{y \geq \delta_{m}\right\}$ and $\hat{V}^{\varepsilon_{m}}(v, \Sigma, m) \backslash\left\{\tilde{y} \geq 2 \delta_{m} /\left\|\mathcal{L}\left(f_{m}\right)\right\|\right\}$. If $f(v)$ is also marked, then we can require $F_{m}$ to preserve the marking. 
If $f(v)$ is a node between two visible components, then $F_{m}$ maps $\hat{V}^{\varepsilon_{m}}\left(v, \Sigma_{m}\right) \backslash$ $\left\{y \geq \delta_{m}\right\}$ onto $\hat{V}^{\varepsilon_{m}}(v, \Sigma, m) \backslash\left\{\tilde{y} \geq 2 \delta_{m} /\left\|\mathcal{L}\left(f_{m}\right)\right\|\right\}$ shrinking the edges $\left\{f_{m}\left(\eta_{i}^{*}\right)\right\}$ to $f(v)$ and as a diffeomorphism elsewhere.

If $\Sigma^{\prime} \subset \Sigma$ is an invisible component and $f\left(v_{1}\right), \ldots, f\left(v_{l}\right)$ are vertices of $f(G) \subset \Sigma_{+}$ and nodes of $\Sigma^{\prime}$, then we require $F_{m}$ to map

$$
\bigcup_{k} \hat{V}^{\varepsilon_{m}}\left(v_{k}, \Sigma_{m}\right) \backslash\left\{y \geq \delta_{m}\right\} \quad \text { onto } \quad \Sigma^{\prime} \cup\left(\bigcup_{k} \hat{V}^{\varepsilon_{m}}\left(v_{k}, \Sigma, m\right) \backslash\left\{\tilde{y} \geq 2 \delta_{m} /\left\|\mathcal{L}\left(f_{m}\right)\right\|\right\}\right)
$$

by shrinking each $\hat{V}^{\varepsilon_{m}}\left(v_{k}, \Sigma_{m}\right) \cap\left\{y=\delta_{m} / 2\right\}$ to $f\left(v_{k}\right)$ and as a diffeomorphism elsewhere.

\section{Table of symbols}

\begin{tabular}{|c|c|}
\hline$S^{\circ}$ & open surface $S \backslash(\partial S \cup\{$ marked points $\})$ \\
\hline$\dot{S}$ & punctured surface $S \backslash\{$ marked points $\}$ \\
\hline$d S$ & double of the surface $S$ \\
\hline$\sigma$ & natural real involutions of $d S$ \\
\hline $\mathbb{H}$ & upper half-plane \\
\hline$\check{\mathcal{T}}(S)$ & space of $S$-marked smooth hyperbolic surfaces \\
\hline $\mathcal{T}(S)$ & Teichmüller space of $S$-marked hyperbolic surfaces with geodesic boundary \\
\hline$\ell, \tau$ & hyperbolic length function and twist parameter \\
\hline $\mathcal{L}$ & boundary length map \\
\hline$\check{\mathcal{T}}(S)(p)$ & locus $\mathcal{L}^{-1}(p) \subset \check{\mathcal{T}}(S)$ of surfaces with boundary lengths $p$ \\
\hline $\mathcal{A}(S)$ & set of $\operatorname{arcs}$ on $S$ \\
\hline $\mathfrak{A}(S)$ & complex of $\operatorname{arcs}$ on $S$ \\
\hline $\mathfrak{A}^{\circ}(S)$ & subset of proper arc systems in $\mathfrak{A}(S)$ \\
\hline$|\boldsymbol{A}|$ & topological realization of the set $\boldsymbol{A}$ \\
\hline$|\mathfrak{A}(S)|$ & topological realization of $\mathfrak{A}(S)$ \\
\hline$\left|\mathfrak{A}^{\circ}(S)\right|$ & subspace of $|\mathfrak{A}(S)|$ corresponding to proper arc systems \\
\hline $\mathcal{M L}(R)$ & space of measured laminations on $R$ \\
\hline $\mathbb{P} \mathcal{M L}(R)$ & space of projective measured laminations on $R$ \\
\hline$i(\cdot, \cdot)$ & geometric intersection product \\
\hline$T_{\gamma}$ & Dehn twist along $\gamma$ \\
\hline $\mathcal{Q}_{\Sigma}$ & space of holomorphic quadratic differentials on $\Sigma$ \\
\hline $\mathcal{B}_{\Sigma}$ & space of harmonic Beltrami differentials on $\Sigma$ \\
\hline$\omega, \eta$ & Weil-Petersson 2-form and Poisson structure \\
\hline$\overline{\mathcal{T}}(S)$ & augmented Teichmüller space \\
\hline$\Gamma(S)$ & mapping class group $\operatorname{Diff}_{+}(S) / \operatorname{Diff}_{0}(S)$ \\
\hline$\overline{\mathcal{M}}(S)$ & moduli space of stable hyperbolic surfaces diffeomorphic to $S$ \\
\hline$a_{i}, s_{i}$ & hyperbolic length $a_{i}$ of the $\operatorname{arc} \alpha_{i}$ and $s_{i}=\cosh \left(a_{i} / 2\right)$ \\
\hline$w_{\boldsymbol{A}}\left(\vec{\alpha}_{i}\right), w_{\boldsymbol{A}}\left(\alpha_{i}\right)$ & width of the oriented $\operatorname{arc} \vec{\alpha}_{i}$ or of the arc $\alpha_{i}$ with respect to the arc system $\boldsymbol{A}$ \\
\hline$t_{\alpha_{i}}^{\infty}(\mathcal{A}(S))$ & $\begin{array}{l}\text { transverse length } T\left(\ell_{\alpha_{i}}\right)=2 \operatorname{arcsinh}\left(1 / \sinh \left(\ell_{\alpha_{i}} / 2\right)\right) \text { of } \alpha_{i} \\
\text { space of bounded maps } \mathcal{A}(S) \rightarrow \mathbb{R}_{>0}\end{array}$ \\
\hline $\mathbb{P}(\mathcal{A}(S))$ & projectivization of $L^{\infty}(\mathcal{A}(S))$ \\
\hline$\|t\|_{\infty}$ & sup-norm of the function $t$ \\
\hline$j$ & embedding of $\mathcal{T}(S)$ in the space of $t$-length functions \\
\hline$\overline{\mathcal{T}}^{a}(S)$ & bordification of arcs of $\mathcal{T}(S)$ \\
\hline$\overline{\mathcal{M}}^{a}(S)$ & compactification of $\operatorname{arcs}$ of $\mathcal{M}(S)$ \\
\hline
\end{tabular}




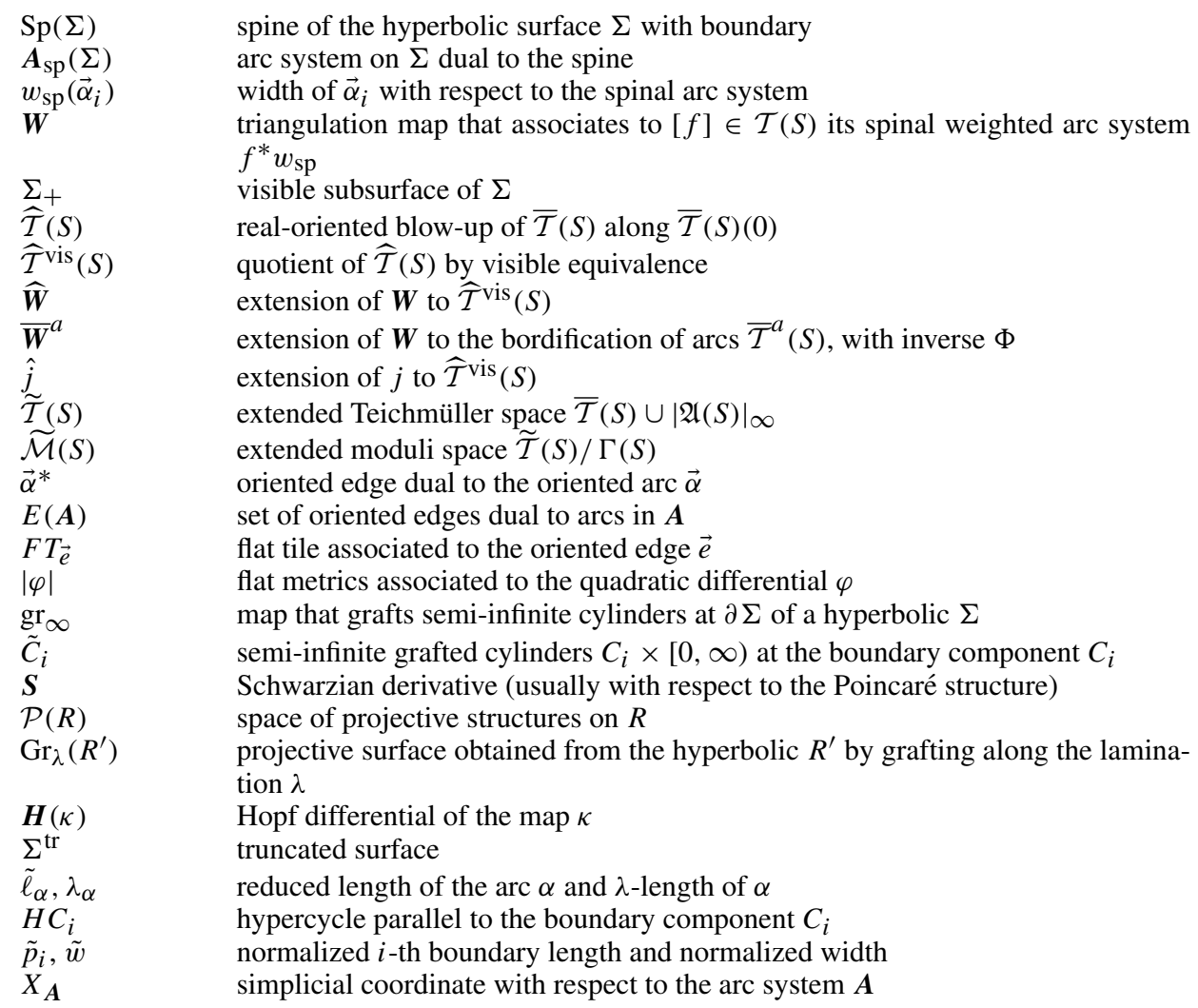

Acknowledgments. I would like to thank Enrico Arbarello, Curtis McMullen, Tomasz Mrówka, Mike Wolf and Scott Wolpert for very fruitful and helpful discussions. I also thank an anonymous referee for useful suggestions and remarks.

\section{References}

[Abi80] Abikoff, W.: The Real Analytic Theory of Teichmüller Space. Lecture Notes in Math. 820, Springer, New York (1980) Zbl 0452.32015 MR 0590044

[AC09] Arbarello, E., Cornalba, M.: Teichmüller space via Kuranishi families. Ann. Scuola Norm. Sup. Pisa Cl. Sci. (5) 8, 89-116 (2009) Zbl 1186.30041 MR 2512202

[ACGH] Arbarello, E., Cornalba, M., Griffiths, P., Harris, J.: Geometry of Algebraic Curves. Volume II. Grundlehren Math. Wiss. 268, Berlin, Springer (2011) Zbl pre05798333

[Ahl61] Ahlfors, L. V.: Some remarks on Teichmüller's space of Riemann surfaces. Ann. of Math. 74, 171-191 (1961) Zbl 0146.30602 MR 0204641

[BP92] Benedetti, R., Petronio, C.: Lectures on Hyperbolic Geometry. Universitext, Springer, Berlin (1992) Zbl 0768.51018 MR 1219310

[Ber74] Bers, L.: Spaces of degenerating Riemann surfaces. In: Discontinuous Groups and Riemann Surfaces (College Park, MD, 1973), Ann. of Math. Stud. 79, Princeton Univ. Press, Princeton, NJ, 43-55 (1974) Zbl 0294.32016 MR 0361051 
[Ber76] Bers, L.: Nielsen extensions of Riemann surfaces. Ann. Acad. Sci. Fenn. Ser. A I Math. 2, 29-34 (1976) Zbl 0352.30014 MR 0486492

[BE88] Bowditch, B. H., Epstein, D. B. A.: Natural triangulations associated to a surface. Topology 27, 91-117 (1988) Zbl 0649.32017 MR 0935529

[DM69] Deligne, P., Mumford, D.: The irreducibility of the space of curves of given genus. Inst. Hautes Études Sci. Publ. Math. 36, 75-109 (1969) Zbl 0181.48803 MR 0262240

[Dum06] Dumas, D.: Grafting, pruning, and the antipodal map on measured laminations. J. Differential Geom. 74, 93-118 (2006) Zbl 1104.32006 MR 2260929

[Dum07a] Dumas, D.: Erratum to: "Grafting, pruning, and the antipodal map on measured laminations”. J. Differential Geom. 77, 175-176 (2007) Zbl 1123.32301 MR 2344358

[Dum07b] Dumas, D.: The Schwarzian derivative and measured laminations on Riemann surfaces. Duke Math. J. 140, 203-243 (2007) Zbl 1134.30035 MR 2359819

[FLP79] Travaux de Thurston sur les surfaces. Astérisque 66-67 (1979) Zbl 0731.57001 MR 0568308

[Har86] Harer, J. L.: The virtual cohomological dimension of the mapping class group of an orientable surface. Invent. Math. 84, 157-176 (1986) Zbl 0592.57009 MR 0830043

[HM79] Hubbard, J., Masur, H.: Quadratic differentials and foliations. Acta Math. 142, 221-274 (1979) Zbl 0415.30038 MR 0523212

[KT92] Kamishima, Y., Tan, S. P.: Deformation spaces on geometric structures. In: Aspects of Low-Dimensional Manifolds, Adv. Stud. Pure Math. 20, Kinokuniya, Tokyo, 263-299 (1992) Zbl 0798.53030 MR 1208313

[Kee74] Keen, L.: Collars on Riemann surfaces. In: Discontinuous Groups and Riemann Surfaces (College Park, MD, 1973), Ann. of Math. Stud. 79, Princeton Univ. Press, Princeton, NJ, 263-268 (1974) Zbl 0304.30014 MR 0379833

[Kon92] Kontsevich, M.: Intersection theory on the moduli space of curves and the matrix Airy function. Comm. Math. Phys. 147, 1-23 (1992) Zbl 0756.35081 MR 1171758

[KS93] Korevaar, N. J., Schoen, R.: Sobolev spaces and harmonic maps for metric space targets. Comm. Anal. Geom. 1, 561-659 (1993) Zbl 0862.58004 MR 1266480

[KP94] Kulkarni, R. S., Pinkall, U.: A canonical metric for Möbius structures and its applications. Math. Z. 216, 89-129 (1994) Zbl 0813.53022 MR 1273468

[Loo95] Looijenga, E.: Cellular decompositions of compactified moduli spaces of pointed curves. In: The Moduli Space of Curves (Texel Island, 1994), Progr. Math. 129, Birkhäuser Boston, Boston, MA, 369-400 (1995) Zbl 0862.14017 MR 1363063

[Luo07] Luo, F.: On Teichmüller spaces of surfaces with boundary. Duke Math. J. 139, 463-482 (2007) Zbl 1138.32006 MR 2350850

[Mas76] Masur, H.: Extension of the Weil-Petersson metric to the boundary of Teichmüller space. Duke Math. J. 43, 623-635 (1976) Zbl 0358.32017 MR 0417456

[Mat76] Matelski, J. P.: A compactness theorem for Fuchsian groups of the second kind. Duke Math. J. 43, 829-840 (1976) Zbl 0341.30020 MR 0432921

[MP07] McShane, G., Penner, R.: Stable curves and screens on fatgraphs. arXiv:0707.1468 (2007)

[Mir07] Mirzakhani, M.: Weil-Petersson volumes and intersection theory on the moduli space of curves. J. Amer. Math. Soc. 20, 1-23 (2007) Zbl 1120.32008 MR 2257394

[Mon09a] Mondello, G.: A criterion of convergence in the augmented Teichmüller space. Bull. London Math. Soc. 41, 733-746 (2009) Zbl 1176.30069 MR 2521369

[Mon09b] Mondello, G.: Riemann surfaces, ribbon graphs and combinatorial classes. In: Handbook of Teichmüller Theory, Vol. II, IRMA Lect. Math. Theor. Phys. 13, Eur. Math. Soc., Zürich, 151-215 (2009) Zbl 1183.30002 MR 2497787 
[Mon09c] Mondello, G.: Triangulated Riemann surfaces with boundary and the Weil-Petersson Poisson structure. J. Differential Geom. 81, 391-436 (2009) Zbl 1165.53026 MR 2472178

[Pen87] Penner, R. C.: The decorated Teichmüller space of punctured surfaces. Comm. Math. Phys. 113, 299-339 (1987) Zbl 0642.32012 MR 0919235

[Pen92] Penner, R. C.: Weil-Petersson volumes. J. Differential Geom. 35, 559-608 (1992) Zbl 0768.32016 MR 1163449

[Rat06] Ratcliffe, J. G.: Foundations of Hyperbolic Manifolds. 2nd ed., Grad. Texts in Math. 149, Springer, New York (2006) Zbl 1106.51009 MR 2249478

[SW02] Scannell, K. P., Wolf, M.: The grafting map of Teichmüller space. J. Amer. Math. Soc. 15, 893-927 (2002) Zbl 1011.32009 MR 1915822

[Str67] Strebel, K.: On quadratic differentials with closed trajectories and second order poles. J. Anal. Math. 19, 373-382 (1967) Zbl 0158.32402 MR 0224808

[Tan97] Tanigawa, H.: Grafting, harmonic maps and projective structures on surfaces. J. Differential Geom. 47, 399-419 (1997) Zbl 0955.32012 MR 1617652

[Ush99] Ushijima, A.: A canonical cellular decomposition of the Teichmüller space of compact surfaces with boundary. Comm. Math. Phys. 201, 305-326 (1999) Zbl 0938.32011 MR 1682230

[Wol89] Wolf, M.: The Teichmüller theory of harmonic maps. J. Differential Geom. 29, 449-479 (1989) Zbl 0655.58009 MR 0982185

[Wol83] Wolpert, S.: On the symplectic geometry of deformations of a hyperbolic surface. Ann. of Math. (2) 117, 207-234 (1983) Zbl 0518.30040 MR 0690844

[Wol85] Wolpert, S.: On the Weil-Petersson geometry of the moduli space of curves. Amer. J. Math. 107, 969-997 (1985) Zbl 0578.32039 MR 0796909 